\title{
NONSMOOTH ANALYSIS: DIFFERENTIAL CALCULUS OF NONDIFFERENTIABLE MAPPINGS
}

\author{
BY
}

A. D. IOFFE

\begin{abstract}
A new approach to local analysis of nonsmooth mappings from one Banach space into another is suggested. The approach is essentially based on the use of set-valued mappings of a special kind, called fans, for local approximation. Convex sets of linear operators provide an example of fans. Generally, fans can be considered a natural set-valued extension of linear operators. The first part of the paper presents a study of fans; the second is devoted to calculus and includes extensions of the main theorems of classical calculus.
\end{abstract}

Introduction. The idea to extend the framework of differential calculus so as to cover more general classes of functions and mappings is by no means new. Basically, it was the underlying idea for the differentiation theory connected with the Lebesgue integral and for the theory of distributions. Both theories deal essentially with what could be called nonlocal aspects of the calculus centered around the Newton-Leibniz and integration by parts formulae. The notion of the value of a derivative at a given point makes no sense in either of them.

Nonsmooth analysis appeared in the 1970's just to carry out an extension of the local aspect of the calculus connected with the idea of (linear) approximation of a mapping about a given point. Certain separate ideas and results appeared of course much earlier (one could recall the Dini numbers for instance) but a systematic study began during the last decade when the natural development of the optimization theory made the need for such an extension very acute and, as often happens, practical and heuristic computations were initiated before an adequate theory appeared (see [52] and references therein).

It is not surprising that the main impulse came from the optimization theory which has natural mechanisms generating nonsmoothness. But as a result, most of the efforts were applied to obtain more and more refined conditions for extrema with less interest in those aspects of analysis that are less immediately connected with this purpose. The only exception was perhaps the generalized gradients of Clarke whose analytical virtues were recognized from the beginning ([8]-[12], [17], [20]-[24], [26], [36], [38], [45], [46], [49], [50], [53], [61] and others).

The original motivation for the present research was just to find a satisfactory extension of Clarke's approach to mappings in infinite-dimensional spaces and the first version of this paper (see [25] for a summary of results) was written completely along these lines. Later, however, it became more and more difficult to ignore a

Received by the editors September 25, 1979 and, in revised form, July 18, 1980.

1980 Mathematics Subject Classification. Primary 26A96; Secondary 26A57. 
very important circumstance, namely that the approximation that generalized gradients provide for is rather rough and that certain other devices, less flexible analytically and less convenient to define constructively as they are, give sometimes more subtle results ([18], [42], [43], [57], [60]).

As a result, this paper appears to be an attempt to develop a general outlook on nonsmooth analysis, on the one hand, and, on the other hand, it presents a more detailed study of certain special classes of approximating objects (within the framework of this general outlook).

A general class of objects chosen here to be the apparatus for local approximation of nonsmooth mappings is formed by closed-valued multifunctions which are positively homogeneous of degree one. The most important point is that, unlike in the classical calculus, it is not a single object that we consider a "derivative", but rather a net of objects called prederivatives which provide a mapping with an ever finer approximation about the point. What would be natural to call derivatives are limit objects of nets and they rarely exist in the general case. (This philosophy is not new. It was clearly expressed by Warga [56] and, for a somewhat different purpose, by Levitin-Miljutin-Osmolovskii [62].)

Most approaches to nonsmooth analysis can be considered special methods to construct prederivatives or define them axiomatically (as in [19]). We shall study here several new classes of prederivatives, among them an extension of Clarke's generalized gradients and Jacobians to an infinite-dimensional setting.

The crucial question we tried to answer while endeavouring to carry out such an extension was: do convex sets of linear operators provide a good approximation for Lipschitz mappings in Banach spaces? The answer proved to be negative and the attempts to find a substitute resulted in introducing convex-valued mappings of a special kind called fans. (I cannot avoid mentioning a funny coincidence: Halkin [19] used the same, not very scientifically sounding, word for different approximating objects which were compact sets of linear operators. Geometrically, the graph of any homogeneous set-valued mapping resembles a deployed fan. This analogy becomes even more noticeable if the graph is decomposed into graphs of linear operators.)

According to the now prevailing terminology, fans should be defined as convexvalued sublinear multifunctions. Linear operators and convex sets of linear operators are examples of fans. And generally, there is a surprising parallelism between properties of fans and linear operators which could be traced throughout the paper.

(It would be wrong to assert that nothing like fans appeared earlier. I know of one work [51] where similar objects were introduced in connection with the Hahn-Banach extension theorem. As a matter of fact, fans seem to be a very natural language for anything connected with extensions of linear operators-see $\$ 7$ here and also [27], [28]. But it was probably Rockafellar who first considered them as early as in the middle 1960's as antipodes for convex processes studied in [48]. His results remained unpublished because, as I understand, no possible application was known at that time.) 
Certain results on fans are collected in Chapter 1. I have been trying to concentrate upon those properties of fans that could be further applied to nonsmooth analysis. Many interesting questions remained, however, outside this scope.

The second chapter is devoted to the differential calculus. It begins with surveying in $\$ 8$ certain definitions and results about various tangent and normal cones. Main definitions are gathered in $\$ 9$. Here general notions of a prederivative, a strict prederivative (with the word "strict" having a meaning close to that in the classical calculus [7]) and a derivative are introduced as well as three special classes of them. In particular, fans appear naturally as, in a sense, a base of the net of all strict prederivatives. The other two classes relate to plus-derivatives like those considered in [45] and to derivatives introduced by Mordukhovich [42] in the same way as fan-prederivatives correspond to generalized gradients of Clarke. $\S 10$ is mainly devoted to describing situations in which the graph of a strict prederivative can be decomposed into graphs of linear operators. To a large extent, this happens when the local behaviour of a Lipschitz mapping can be described by a set of linear operators. In $\$ 11$ we consider various surjection theorems and their corollaries (Ljusternik-type theorems, inverse mapping theorems, implicit function theorems). They seem to be the first infinite-dimensional results which contain their classical smooth counterparts in the most general form. The final 12th section contains several examples which have been added mainly to demonstrate that calculations involving fans are almost as easy as with the usual derivatives of smooth mappings.

Structurally, the differentiation theory using fans is very similar to the classical calculus. As there, we have an independently defined approximation apparatus and a device to transform properties of approximations into the corresponding properties of the mappings to be approximated. In the classical calculus this used to be done with the help of various fixed point theorems, especially of the contraction mapping principle. In the nonsmooth case, this principle does not work. Instead we have the variational principle of Ekeland [16] which proved to be completely adequate to the situation.

We deal here only with single-valued mappings and refer to [3], [15], [41] for differentiation results concerning set-valued mappings. Some of what will be said here can also be applied to them. But so far a satisfactory version of the techniques we use here which would be completely applicable to set-valued mappings has not been found.

This paper, as well as some of my earlier papers, has been prepared for publication in rather unusual circumstances with normal connection between the publisher and the author almost completely broken. The patience and goodwill of the Transactions are thankfully acknowledged. I doubt that the paper could appear at all without the generous cooperation of Professor Terry Rockafellar and Professor Jack Warga which included reading, correcting errors, inserting changes and proofreading. I have no words to express my gratitude to them. I am also greatly thankful to Professor Jean-Pierre Aubin for stimulating discussions on the subject (by correspondence) and for constant friendly encouragement. 
Notation. $X, Y, Z$, if nothing else is said, are locally convex Hausdorff topological vector spaces over reals;

$X^{*}$ is the space dual to $X$;

$X_{s}^{*}, X_{w}^{*}$ are the strong and weak dual spaces;

$\left\langle x^{*}, x\right\rangle$ is the canonical pairing between $X$ and $X^{*}$;

$\bar{C}$ is the closure of $C$;

$\overline{\text { conv }} C$ is the convex closure of $C$;

$\|x\|$ is the norm in a Banach space $X$;

$U_{X}, B_{X}$ are open and closed unit balls in a Banach space $X$;

$U(x, r)$ is the open ball of radius $r$ about $x$;

$B(x, r)$ is the closed ball of radius $r$ about $x$;

$\rho(x, C)$ is the distance from the point $x$ to $C$;

$H(C, D)$ is the Hausdorff distance between $C$ and $D$;

$\delta^{*}\left(x^{*}, C\right)$ is the support function of $C$;

$\partial f(x)$ is the subdifferential of a convex function $f$ at $x$.

1. FANS AND BISUBLINEAR FUNCTIONS

1. Definitions and elementary properties. All set-valued mappings, if nothing is added, are assumed closed-valued.

1.1. Defintion. Let $\mathcal{Q}$ be a set-valued mapping from $X$ into $Y$. It is called homogeneous if

(1.1.1) $0 \in \mathbb{Q}(0)$,

(1.1.2) $\mathbb{Q}(\lambda x)=\lambda \mathscr{Q}(x)$ for all $x$ and $\lambda>0$.

It follows that $\mathscr{Q}(0)$ is a pointed cone. The sets $\operatorname{dom} \mathbb{Q}=\{x \mid \mathbb{Q}(x) \neq \varnothing\}$ and supp $\mathbb{Q}=\{x \in \operatorname{dom} \mathbb{Q} \mid \mathbb{Q}(x) \neq Y\}$ will be called the domain and support of $\mathbb{Q}$.

A homogeneous set-valued mapping $Q$ will be called a prefan if

(1.1.3) all sets $Q(x)$ are convex.

A prefan $\mathscr{Q}$ will be called a fan if

(1.1.4) $\mathbb{Q}(x+u) \subset \overline{\mathbb{Q}(x)+\mathbb{Q}(u)}$ for all $x, u \in X$.

1.2. Definition. Let $\mathcal{Q}$ be a set-valued mapping from $X$ into $Y$. The extendedreal-valued function

$$
s_{\mathscr{Q}}\left(y^{*}, x\right)=\delta^{*}\left(y^{*}, \mathbb{Q}(x)\right)=\sup \left\{\left\langle y^{*}, y\right\rangle \mid y \in \mathbb{Q}(x)\right\}
$$

will be called the support function of $\mathscr{Q}$. As usual, we set sup $\varnothing=-\infty$. Often, if this does not cause any confusion, we omit the subscript $\mathbb{Q}$.

1.3. Definition. An extended-real-valued function $f(x, y)$ on $X \times Y$ will be called bisublinear if it is sublinear (i.e. convex and positively homogeneous of degree one) in each variable.

1.4. Proposition. An extended-real-valued function $s\left(y^{*}, x\right)$ on $Y^{*} \times X$ is the support function of a fan $\mathbb{Q}$ from $X$ into $Y$ if and only if it is a bisublinear function such that $s\left(y^{*}, x\right)=-\infty$ if $x \notin \operatorname{dom} Q$ and $s(\cdot, x)$ is $\sigma\left(Y^{*}, Y\right)$-lower semicontinuous and everywhere more than $-\infty$ if $x \in \operatorname{dom} \mathbb{Q}$. In this case

$$
\mathcal{Q}(x)=\left\{y \in Y\left|s\left(y^{*}, x\right)\right\rangle\left\langle y^{*}, y\right\rangle, \forall y^{*} \in Y^{*}\right\} .
$$

Proof. This is an elementary exercise in convex analysis [30], [48]. 
1.5. Definition. Let $L^{*} \subset Y^{*}, K \subset X$ be pointed convex cones. A fan $\mathbb{Q}$ will be called an $L^{*}$-oriented fan supported on $K$ if $\operatorname{dom} Q=X$, supp $Q=K$ and the support function of $Q$ differs from infinity precisely on $L^{*} \times K$. The notion of orientation will not be used in this paper except for examples in the next section. But it is useful for fans with values in ordered spaces [27].

1.6. Definition. A homogeneous set-valued mapping is odd if $\mathscr{Q}(-x)=-\mathbb{Q}(x)$ for all $x$. This amounts to saying that $\mathscr{Q}(\lambda x)=\lambda \mathscr{Q}(x)$ for all $\lambda \neq 0$.

1.7. Proposition. A prefan $\mathcal{Q}$ from $X$ into $Y$ is odd if and only if

$$
s\left(y^{*},-x\right)=s\left(-y^{*}, x\right) \text { for all } y^{*}, x .
$$

Proof. This is a reformulation of a well-known property of support functions of sets: $\delta^{*}\left(y^{*},-C\right)=\delta^{*}\left(-y^{*}, C\right)$.

Unlike the boundedness property introduced below, the oddness assumption enters statements of rather few theorems here. But, as we shall see, all the most important fans appear to be odd.

1.8. Definition. A homogeneous set-valued mapping $\mathscr{Q}$ from $X$ into $Y$ is bounded if $\mathscr{Q}(x) \neq \varnothing$ for all $x$ and for any neighbourhood $V$ about the origin in $Y$ there is a neighbourhood $U$ about the origin in $X$ such that $\mathscr{Q}(x) \subset V$ whenever $x \in U$.

1.9. Definition. Let $\mathcal{Q}$ be a fan with nonempty values. If $s\left(y^{*}, x\right)$ is $\sigma\left(X, X^{*}\right)$ 1.s.c. in $x$ then the set-valued mapping $\mathbb{Q}^{*}\left(y^{*}\right)=\left\{x^{*} \in X^{*}\left|s\left(y^{*}, x\right)\right\rangle\left\langle x^{*}, x\right\rangle\right.$, $\forall x \in X\}$ is a fan from $Y^{*}$ into $X^{*}$ which will be called the adjoint to $\mathcal{Q}$. If $\mathbb{Q}$ is $L^{*}$-oriented and supported on $K$, then $Q^{*}$ is $K$-oriented and supported on $L^{*}$. Obviously, no adjoint can be in general associated with a prefan which is not a fan.

1.10. Proposition. Let $\mathbb{Q}$ be a fan from $X$ into $Y$. Assume that the adjoint fan $\mathbb{Q}^{*}$ exists. Then $Q^{*}$ is weak* lower semicontinuous in the following sense: for any $v^{*} \in Y^{*}$ and any weak open set $U \subset X^{*}$ containing the origin, there is a weak* open set $V \subset Y^{*}$ containing $v^{*}$ such that $\mathbb{Q}^{*}\left(v^{*}\right) \subset \mathbb{Q}^{*}\left(y^{*}\right)+U$ for any $y^{*} \in V$.

Proof. Take $x_{1}, \ldots, x_{n} \in X$ and $\varepsilon>0$ such that $U\left(x_{1}, \ldots, x_{n}, \varepsilon\right)=$ $\left\{x^{*}||\left\langle x^{*}, x_{i}\right\rangle \mid<\varepsilon, i=1, \ldots, n\right\} \subset U$. Since $s(\cdot, x)$ is weak* lower semicontinuous, the set $V=\left\{y^{*} \mid s\left(y^{*}, \pm x_{i}\right)>s\left(v^{*}, \pm x_{i}\right)-\varepsilon, i=1, \ldots, n\right\}$ is nonempty (it contains $\left.v^{*}\right)$ and open in the weak* topology.

Fix $y^{*} \in V$ and $x^{*} \in Q^{*}\left(y^{*}\right)$ and consider the function $f(x)=s\left(y^{*}, x\right)-$ $\left\langle x^{*}, x\right\rangle$. This function is everywhere finite, sublinear and weakly lower semicontinuous. We have also $f\left( \pm x_{i}\right)>-\varepsilon$. Let $z^{*} \in \partial f(0)$ be such that $\left\langle z^{*}, \pm x_{i}\right\rangle\langle-\varepsilon$ for any $i=1, \ldots, n$. (Such a $z^{*}$ does exist. Indeed, consider two sets in $X \times R$ : epi $f=\{(x, a) \mid a \geqslant f(x)\}$, the epigraph of $f$, and $\left.C=\left(\operatorname{conv}\left\{ \pm x_{1}, \ldots, \pm x_{n}\right\}\right),-\varepsilon\right)$. The first of them is closed, the second is compact and they do not meet each other. Take a hyperplane separating these sets and use the fact that $f$ is everywhere finite which makes it impossible for the hyperplane to be vertical.) Then $\left|\left\langle z^{*}, \pm x_{i}\right\rangle\right|\langle\varepsilon$ for all $i$ and hence $z^{*} \in U\left(x_{1}, \ldots, x_{n}, \varepsilon\right)$.

On the other hand, $f$ is the sum of two sublinear functions, one of which is weakly l.s.c. and the other of which is linear and continuous. Therefore $\partial f(0)$ is the 
sum of the subdifferentials of the summands, i.e. $\partial f(0)=\partial_{x} s\left(y^{*}, 0\right)-x^{*}$ which means that there is $w^{*} \in \partial_{x} s\left(y^{*}, 0\right)=\mathbb{Q}^{*}\left(y^{*}\right)$ such that $x^{*}=w^{*}+z^{*}$. Hence $x^{*} \in \mathbb{Q}^{*}\left(y^{*}\right)+U\left(x_{1}, \ldots, x_{n}, \varepsilon\right)$.

1.11. Proposition. If $\mathbb{Q}$ is an odd fan and $\mathbb{Q}^{*}$ exists, then $\mathbb{Q}^{*}$ is also an odd fan.

Proof. This is obvious.

1.12. Proposition. Let $\mathbb{Q}$ be a bounded fan from $X$ into $Y$. Then $s\left(y^{*}, x\right)$ is continuous in $x$ for any $y^{*} \in Y^{*}$ and the set-valued mapping $x \rightarrow \mathbb{Q}(x)$ is continuous in the following sense: for any open $V \subset Y$ containing the origin there is an open set $U \subset X$ also containing the origin such that $\mathbb{Q}(x) \subset \mathbb{Q}\left(x^{\prime}\right)+V$ whenever $x-x^{\prime} \in$ $U$.

Proof. Given $y^{*}$, we can choose a neighbourhood $U$ about the origin in $X$ in such a way that $\left\langle y^{*}, y\right\rangle \leqslant 1$ whenever $y \in \mathbb{Q}(x), x \in U$. Then $s\left(y^{*}, x\right)<1$ for $x \in U$. Hence $s\left(y^{*}, \cdot\right)$ is continuous on $U$ (as a convex function bounded from above on an open set [6]) and hence on all of $X$ since $0 \in U$ and $s\left(y^{*},{ }^{\cdot}\right)$ is homogeneous.

Now if $U$ is such that $Q(x) \subset(1-\varepsilon) V$ for $x \in U$, then, whenever $x-x^{\prime} \in U$,

$$
\mathbb{Q}(x) \subset \overline{\mathbb{Q}\left(x^{\prime}\right)+\mathbb{Q}\left(x-x^{\prime}\right)} \subset \overline{\mathbb{Q}\left(x^{\prime}\right)+(1-\varepsilon) V} \subset \mathbb{Q}\left(x^{\prime}\right)+V \text {. }
$$

1.13. Corollary. Any bounded fan from $X$ into $Y$ has an adjoint which is a bounded fan from $Y_{s}^{*}$ into $X_{w}^{*}$ with $\sigma\left(X^{*}, X\right)$-compact values.

Proof. The existence of $\mathbb{Q}^{*}$ follows from 1.9. All sets $\mathbb{Q}^{*}\left(y^{*}\right)$ are $\sigma\left(X^{*}, X\right)$-compact as subdifferentials of continuous convex functions $x \rightarrow s\left(y^{*}, x\right)$ at the origin.

Thus only boundedness needs to be proved. Since all sets $\mathscr{Q}(x)$ are bounded, the function $y^{*} \rightarrow s\left(y^{*}, x\right)$ is continuous on $Y_{s}^{*}$ for any $x \in X$. Let $\varepsilon>0$ and

$$
\begin{gathered}
U\left(x_{1}, \ldots, x_{n}, \varepsilon\right)=\left\{x^{*} \in X^{*}||\left\langle x^{*}, x_{i}\right\rangle \mid<\varepsilon, i=1, \ldots, n\right\}, \\
Q_{i}=\left\{y^{*} \in Y^{*} \mid s\left(y^{*}, x_{i}\right)<1, s\left(y^{*},-x_{i}\right)<1\right\} .
\end{gathered}
$$

The sets $Q_{i}$ are open in the strong topology of $Y^{*}$ and contain the origin. Thus so is $Q=\cap Q_{i}$ and, whenever $y^{*} \in \varepsilon Q$, we have $\mathbb{Q}^{*}\left(y^{*}\right) \subset\left\{x^{*}||\left\langle x^{*}, x_{i}\right\rangle \mid<\varepsilon, i=\right.$ $1, \ldots, n\} \subset U\left(x_{1}, \ldots, x_{n}, \varepsilon\right)$.

1.14. Definition. In an obvious way, the notion of a bisublinear function can be extended to the case of many variables. Namely, an extended-real-valued function $s\left(y_{1}^{*}, \ldots, y_{k}^{*}, x_{1}, \ldots, x_{n}\right)$ will be called a polysublinear function of type $(k, n)$ if it is sublinear in each variable. Most important for us are polysublinear functions of type $(1, n)$ which are naturally connected with set-valued mappings.

A set-valued mapping $\mathcal{Q}$ from $X^{n}$ into $Y$ will be called an $n$-fan if all sets $\mathcal{Q}\left(x_{1}, \ldots, x_{n}\right)$ are nonempty, convex and closed and the support function of $\mathbb{Q}$ is a polysublinear function of type $(1, n)$. The notions of oddness and boundedness can be easily extended to polyfans.

In this paper we shall deal only with 2 -fans. Such a fan will be called symmetric if $\mathbb{Q}\left(x_{1}, x_{2}\right)=\mathbb{Q}\left(x_{2}, x_{1}\right)$. Clearly, a 2-fan is symmetric if and only if $s\left(y^{*}, x_{1}, x_{2}\right)=$ $s\left(y^{*}, x_{2}, x_{1}\right)$ for all values of the variables. 
2. Examples. In this section we shall show that the class of fans includes all homogeneous objects of convex analysis: linear operators, convex sets of linear operators, convex processes, sublinear operators. Later in $\$ 7$ and Chapter 2 we shall see that the class of fans is, in fact, much richer.

2.1. Fans generated by sets of linear operators. Any linear operator is a fan (from all of $X$ ) and any fan whose values are singletons is a linear operator. This is obvious.

More generally, let $\mathfrak{U}$ be a convex weakly closed set of linear operators from $X$ into $Y$. Consider the set-valued mapping $x \rightarrow \mathbb{Q}(x)=\{y \in Y \mid y=A x$ for some $A \in \mathfrak{A}\}$. It is not difficult to see that $\mathcal{Q}$ is a fan and that, moreover, in this case $\mathbb{Q}(x+u) \subset \mathbb{Q}(x)+\mathbb{Q}(u)$.

We shall say that $\mathcal{Q}$ is generated by $\mathfrak{A}$. It is obvious that a fan generated by a set of operators is odd.

The support function of $\mathcal{Q}$,

$$
s\left(y^{*}, x\right)=\sup \left\{\left\langle y^{*}, A x\right\rangle \mid A \in \mathfrak{A}\right\},
$$

is both $\sigma\left(Y^{*}, Y\right)$-1.s.c. in $y^{*}$ and $\sigma\left(X, X^{*}\right)$-1.s.c. in $x$ so that $\mathbb{Q}$ has an adjoint. A simple calculation shows that the adjoint fan is defined as follows:

$$
\mathbb{Q}^{*}\left(y^{*}\right)=\left\{x^{*} \in X^{*} \mid x^{*}=A^{*} y^{*} \text { for some } A \in \mathfrak{A}\right\} .
$$

Thus $\mathbb{Q}^{*}$ is generated by $\mathfrak{U}^{*}$, the set of operators adjoint to elements of $\mathfrak{A}$.

In view of what has been said in the introduction, the problem of characterizing fans generated by sets of operators is most important. We shall discuss it more thoroughly in $§ 7$. A simple example of a fan not generated by a set of operators is the following: let $X=R^{2}$, let $e_{1}, e_{2}$ be a basis in $X$, let $Y$ be a nonreflexive Banach space, and let $C_{1}$ and $C_{2}$ be two closed bounded subsets of $Y$ whose sum is not closed. Then we set

$$
\mathcal{Q}\left(\lambda e_{1}+\mu e_{2}\right)=\lambda C_{1}+\mu C_{2} .
$$

2.2. Linear system. According to the terminology accepted in the system theory [39], a linear system is a set-valued mapping $\mathcal{L}$ from $X$ into $Y$ such that $\mathcal{L}(x)+$ $\mathfrak{L}(u) \subset \mathcal{L}(x+u)$ and $\mathcal{L}(\lambda x)=\lambda \mathscr{L}(x)$ if $\lambda \neq 0$.

It is easy to see that $\mathcal{L}$ is a linear system if and only if the second of the relations above holds and there is a subspace $L \subset Y$ such that $\mathcal{L}(x)=\mathcal{L}(x)+L$ for every $x$. Therefore we have actually $\mathcal{L}(x)+\mathcal{L}(u)=\mathscr{L}(x+u)$ and hence any linear system with closed values is a fan with orientation defined by a certain $\sigma\left(Y^{*}, Y\right)$ closed subspace $L^{*} \subset Y^{*}$. The support function of this fan is bilinear on $L^{*} \times X$; hence the adjoint fan is defined by a linear mapping from $L^{*}$ into $X^{*}$.

2.3. Restrictions of fans and convex processes. Given a fan $\mathcal{Q}$ and a pointed cone $K \subset X$, we can define the restriction $\mathcal{Q}_{K}$ of $\mathcal{Q}$ to $K$ as the fan whose support function is $s\left(y^{*}, x\right)+\delta(x, K)$, where $\delta(x, K)$ is the indicator function of $K$, i.e. the one equal to zero on $K$ and $+\infty$ outside.

Clearly $\operatorname{supp} \mathbb{Q}_{K}=(\operatorname{supp} \mathbb{Q}) \cap K$. If $K^{\circ}$ is the polar cone to $K$, then (if $\mathbb{Q}^{*}$ exists)

$$
\left(\mathbb{Q}_{K}\right)^{*}\left(y^{*}\right)=\overline{\mathbb{Q}^{*}\left(y^{*}\right)+K^{\circ}} .
$$


In particular, if $\mathcal{Q}$ is a bounded fan, then, as follows from 1.13,

$$
\left(\mathbb{Q}_{K}\right)^{*}\left(y^{*}\right)=\mathbb{Q} *\left(y^{*}\right)+K^{\circ} \text {. }
$$

It follows that with every pointed convex cone $K \subset X$ we can associate two fans from $K$ into $X$, the restrictions to $K$ of the fans generated by the zero and identity operators.

Convex processes introduced by Rockafellar [48] are by definition set-valued mappings whose graphs are pointed convex cones. Therefore we can associate a fan with every convex process, i.e., at least formally, any convex process can be considered a fan. This reduction seems, however, to be somewhat artificial and it may be that certain properties of convex processes cannot be naturally derived from the general properties of fans. (The interrelation is similar to that between convex functions and their epigraphs. Though the epigraph defines the convex function completely, in most cases considering the function as such is much more natural.)

2.4. Fans associated with sublinear operators. Let $L$ be a closed convex cone in $Y$. Denote by $>$ the partial ordering defined by this cone. A mapping $P: X \rightarrow Y$ is called $L$-sublinear if $P(\lambda x)=\lambda P(x)$ for $\lambda>0$ and $P(x+u) \prec P(x)+P(u)$.

Let $L^{*}=\left\{y^{*} \in Y^{*} \mid\left\langle y^{*}, y\right\rangle>0, \forall y \in L\right\}$, and let an $L$-sublinear mapping $P$ from $X$ into $Y$ be given. Then the set-valued mapping

$$
x \rightarrow \mathbb{Q}(x)=\{y \in Y \mid y<P(x)\}
$$

is an $L^{*}$-oriented fan from $X$ into $Y$.

If $Y$ is a lattice ordered by $L$, then

$$
\mathcal{Q}(x)=[-P(-x), P(x)]=\{y \mid-P(-x) \prec y \prec P(x)\}
$$

is an odd fan and, more generally, if $Q: X \rightarrow Y$ is superlinear (i.e. $-Q$ is sublinear) and $Q(x) \prec P(x)$ for all $x$, then $Q(x)=[Q(x), P(x)]=\{y \mid Q(x) \prec y \prec P(x)\}$ is a fan.

Either of these three fans may fail to be generated by a set of linear operators (cf. [13], [37]) unless the order defined by $L$ is conditionally complete. We refer to [27], [28] for further properties of fans associated with sublinear operators. It is appropriate to mention here that certain extension problems for linear operators, say, the norm preserving extension problem [44] or the majorized extension problem [5], [55] can be formulated naturally and in a unified fashion in terms of fans.

\section{Operations with prefans.}

3.1. Definition. Homogeneous set-valued mappings and prefans can undergo the same operations as other set-valued and convex-valued mappings respectively. Namely, we define

the product $\lambda Q$ of a homogeneous set-valued mapping $Q$ and $\lambda \in R, \lambda \neq 0$, by

$$
(\lambda \mathscr{Q})(x)=\lambda \mathscr{Q}(x)
$$

and set $(0 \mathscr{Q})(x)=\mathbb{Q}(0)$ for all $x$;

the sum of homogeneous set-valued mappings $Q$ and $\mathscr{B}$ by

$$
(\mathscr{Q}+\mathscr{B})(x)=\overline{Q(x)+\mathscr{B}(x)} ;
$$


the Cartesian product of homogeneous set-valued mappings $\mathcal{Q}$ and $\mathscr{B}$ (from $X$ into $Y$ and $Z$ respectively) by

$$
(\mathscr{Q} \times \mathscr{B})(x)=\mathscr{Q}(x) \times \mathscr{B}(x) ;
$$

the direct sum of homogeneous set-valued mappings $\mathcal{Q}$ and $\mathscr{B}$ (from $X$ and $Y$ respectively into $Z$ ) by

$$
(\mathscr{Q} \oplus \mathscr{B})(x, y)=\overline{\mathbb{Q}(x)+\mathscr{B}(y)} ;
$$

the upper bound of prefans $\mathscr{Q}$ and $\mathscr{B}$ (both from $X$ into $Y$ ) by

$$
(\mathscr{Q} \vee \mathscr{B})(x)=\overline{\operatorname{conv}}(\mathscr{Q}(x) \cup \mathscr{B}(x)) ;
$$

and the composition of prefans $\mathbb{Q}$ and $\mathscr{B}$ (from $X$ into $Y$ and from $Y$ into $Z$ respectively) by

$$
(\mathscr{B} \circ \mathscr{Q})(x)=\overline{\operatorname{conv}}\{\bigcup \mathscr{B}(y) \mid y \in \mathbb{Q}(x)\}
$$

3.2. Proposition. We have

$$
\begin{aligned}
s_{\lambda \mathfrak{Q}}\left(y^{*}, x\right) & =|\lambda| s_{\mathscr{Q}}\left((\operatorname{sign} \lambda) y^{*}, x\right), \quad \lambda \neq 0 ; \\
s_{\mathfrak{Q}+\mathscr{B}}\left(y^{*}, x\right) & =s_{\mathscr{Q}}\left(y^{*}, x\right)+s_{\mathscr{B}}\left(y^{*}, x\right) ; \\
s_{\mathscr{Q} \times \mathscr{B}}\left(\left(y^{*}, z^{*}\right), x\right) & =s_{\mathscr{Q}}\left(y^{*}, x\right)+s_{\mathscr{B}}\left(z^{*}, x\right) ; \\
s_{\mathscr{Q} \oplus \mathscr{B}}\left(z^{*},(x, y)\right) & =s_{\mathscr{Q}}\left(z^{*}, x\right)+s_{\mathscr{B}}\left(z^{*}, y\right) ; \\
s_{\mathscr{Q} \vee \mathscr{B}}\left(y^{*}, x\right) & =\max \left\{s_{\mathscr{Q}}\left(y^{*}, x\right), s_{\mathscr{B}}\left(y^{*}, x\right)\right\} ; \\
s_{\mathscr{B} \circ \mathbb{Q}}\left(z^{*}, x\right) & =\sup \left\{s_{\mathscr{B}}\left(z^{*}, y\right) \mid y \in \mathbb{Q}(x)\right\} .
\end{aligned}
$$

If the prefans involved are bounded (odd) then so is the result of every operation; if in addition they are fans, then the result is also a fan.

Proof. Elementary.

3.3. Proposition. For bounded fans, the following formulas hold:

$$
\begin{aligned}
& (\lambda \mathscr{Q})^{*}=\lambda Q^{*} ; \quad(\mathscr{Q}+\mathscr{B})^{*}=\mathscr{Q}^{*}+\mathscr{B}^{*} ; \\
& (\mathscr{Q} \times \mathscr{B})^{*}=Q^{*} \oplus \mathscr{B}^{*} ; \quad\left(\mathscr{Q} \oplus \mathscr{B}^{*}=Q^{*} \times \mathscr{B}^{*} ;\right. \\
& (\mathscr{Q} \vee \mathscr{B})^{*}=Q^{*} \vee \mathscr{B}^{*} ; \quad(\mathscr{Q} \circ \mathbb{Q})^{*}=Q^{*} \circ \mathscr{B}^{*} \text {. }
\end{aligned}
$$

Proof. All formulas are obvious except maybe the last one. To prove it, take $y^{*} \in \mathscr{B}^{*}\left(z^{*}\right)$. Then $\left.s_{\mathscr{B}}\left(z^{*}, y\right)\right\rangle\left\langle y^{*}, y\right\rangle$ for all $y \in Y$.

Hence, given any $x \in X$, we have

$$
\sup \left\{s_{\mathscr{B}}\left(z^{*}, y\right) \mid y \in \mathbb{Q}(x)\right\}>\sup \left\{\left\langle y^{*}, y\right\rangle \mid y \in \mathbb{Q}(x)\right\}=s_{\mathscr{Q}}\left(y^{*}, x\right) .
$$

This is true for any $y^{*} \in \mathscr{B}^{*}\left(z^{*}\right)$. Therefore, in view of 3.2 ,

$$
\begin{aligned}
s_{\mathbb{Q}^{*} \circ \mathscr{B}^{*}}\left(z^{*}, x\right) & =\sup \left\{s_{\mathscr{Q}}\left(y^{*}, x\right) \mid y^{*} \in \mathscr{B}^{*}\left(z^{*}\right)\right\} \\
& <\sup \left\{s_{\mathscr{B}}\left(z^{*}, y\right) \mid y \in \mathscr{Q}(x)\right\}=s_{\mathscr{B} \circ \mathbb{Q}}\left(z^{*}, x\right) .
\end{aligned}
$$

Repeating the same with $\mathscr{B}^{*}$ replaced by $\mathcal{Q}$ and $y^{*}$ by $y$, we obtain the opposite inequality. 
4. The topological space $\mathscr{F}(X, Y)$.

4.1. Definition. We denote by $\mathscr{F}(X, Y)$ the collection of all bounded fans from $X$ into $Y$. According to $\S 3, \mathscr{F}(X, Y)$ is a semigroup with respect to summation, and scalar multiplication is an external operation in $\mathscr{F}(X, Y)$. It is easy to see that $\mathscr{F}(X, Y)$ is a semigroup with reduction, that is to say, $Q+C=\mathscr{B}+\mathcal{C}$ implies $\mathscr{Q}=\mathscr{B}$. In other words, $\mathscr{F}(X, Y)$ is a vector prespace according to the terminology of Akilov and Kutateladze [1].

We begin with supplying $\mathscr{F}(X, Y)$ with "locally convex" topologies which will be done precisely in the same manner as for spaces of linear operators.

4.2. Definition. Given a bounded set $M \subset X$ and a neighbourhood $V \subset Y$ about the origin, we set

$$
\rho_{M, V}(\mathbb{Q}, \mathscr{B})=\sup \left\{\left|s_{\mathscr{Q}}\left(y^{*}, x\right)-s_{\mathscr{B}}\left(y^{*}, x\right)\right| \mid x \in M, y^{*} \in V^{0}\right\},
$$

where $V^{0}=\left\{y^{*} \in Y^{*} \mid\left\langle y^{*}, y\right\rangle \leqslant 1, \forall y \in V\right\}$ is the polar of $V$.

We have $\rho_{M, V}(\mathcal{Q}, \mathscr{B})<\infty$ for all $\mathcal{Q}, \mathscr{B} \in \mathscr{F}(X, Y)$. Indeed, take a neighbourhood $U \subset X$ about the origin such that $\mathcal{Q}(x) \subset V$ whenever $x \in U$. Since $M$ is bounded, there is $\lambda>0$ such that $\lambda M \subset U$ and after simple calculations we obtain $\rho_{M, V}(Q, 0)<1 / \lambda$. It remains to note that

$$
\rho_{M, V}(\mathscr{Q}, \mathscr{B}) \leqslant \rho_{M, V}(\mathscr{Q}, 0)+\rho_{M, V}(\Re, 0)
$$

(here 0 denotes the zero fan).

It is easy to see that any $\rho_{M, V}$ is a pseudometric on $\mathscr{F}(X, Y)$, i.e. it is nonnegative and

$$
\begin{aligned}
& \rho_{M, V}(\mathbb{Q}, \mathscr{Q})=0, \quad \rho_{M, V}(\mathbb{Q}, \mathscr{B})=\rho_{M, V}(\mathscr{B}, \mathbb{Q}), \\
& \rho_{M, V}(\mathbb{Q}, \mathcal{C}) \leqslant \rho_{M, V}(\mathbb{Q}, \mathscr{B})+\rho_{M, V}(\mathscr{B}, \mathcal{C}) .
\end{aligned}
$$

4.3. Proposition. Let $\sigma$ be a family of bounded subsets of $X$ which covers $X$ and contains finite unions. Let $\vartheta$ be a base of neighbourhoods of the origin in $Y$ (formed by convex neighbourhoods). Then the family of pseudometrics $\rho_{M, V}(M \in \sigma, V \in \vartheta)$ defines a locally convex Hausdorff topology in $\mathscr{F}(X, Y)$ which is compatible with the structure of vector prespace in $\mathscr{F}(X, Y)$.

Proof. We need to show that

(a) for any $\mathcal{Q} \in \mathscr{F}(X, Y), M_{i} \in \sigma, V_{i} \in \vartheta, i=1, \ldots, n$, there are $M \in \sigma$ and $V \in \vartheta$ such that

$$
\rho_{M, V}(\mathscr{Q}, \mathscr{B}) \geqslant \max _{i} \rho_{M_{i}, V_{i}}(\mathbb{Q}, \mathscr{B}), \quad \forall \mathscr{B} \in \mathscr{F}(X, Y) ;
$$

(b) for any distinct $\mathcal{Q}, \mathscr{B} \in \mathscr{F}(X, Y)$, there are $M \in \sigma, V \in \vartheta$ such that $\rho_{M, V}(\mathbb{Q}, \mathscr{B})>0$

(c) for any $\lambda_{0}>0, \mathbb{Q}_{0} \in \mathscr{F}(X, Y), M \in \sigma, V \in \vartheta, \delta>0$, there is $\varepsilon>0$ such that $\left|\lambda-\lambda_{0}\right|<\varepsilon, \rho_{M, V}\left(\mathbb{Q}, \mathbb{Q}_{0}\right)<\varepsilon \Rightarrow \rho_{M, V}\left(\lambda \mathscr{Q}, \lambda_{0} \mathscr{Q}_{0}\right)<\delta$;

(d) for any $\mathscr{Q}_{0}, \mathscr{B}_{0} \in \mathscr{F}(X, Y), M \in \sigma, V \in \vartheta, \delta>0$, there is $\varepsilon>0$ such that $\rho_{M, V}\left(\mathbb{Q}, \mathbb{Q}_{0}\right)<\varepsilon, \rho_{M, V}\left(\mathscr{B}, \mathscr{B}_{0}\right)<\varepsilon \Rightarrow \rho_{M, V}\left(\mathbb{Q}+\mathscr{B}, \mathbb{Q}_{0}+\mathscr{B}_{0}\right)<\delta$.

To prove (a), let us take $M \in \sigma$ containing $\cup M_{i}$ and $V \in \vartheta$ which is contained in $\cap V_{i}$; to prove (b), let us choose $x \in X$ and $y^{*} \in Y^{*}$ such that $s_{Q}\left(y^{*}, x\right) \neq$ $s_{\mathscr{B}}\left(y^{*}, x\right)$ and then take any $M \in \sigma$ containing $x$ and $V \in \vartheta$ whose polar contains 
an element positively proportional to $y^{*}$. The proof of (c) follows from the obvious inequality

$$
\rho_{M, V}\left(\lambda \mathscr{Q}, \lambda_{0} \mathscr{Q}_{0}\right)<\lambda \rho_{M, V}\left(\mathbb{Q}, \mathbb{Q}_{0}\right)+\left|\lambda-\lambda_{0}\right| \rho_{M, V}\left(\mathbb{Q}_{0}, 0\right) .
$$

The proof of (d) is similar; one should only take 3.2 into account. It follows also from 3.2 that $\rho_{M, V}(Q, \mathscr{B})$ is a convex function in each argument (in an obvious sense) and hence the topology defined by these pseudometrics is locally convex in the sense that any bounded fan has a base of neighbourhoods in $\mathscr{F}(X, Y)$ formed by convex sets.

4.4. Definition. In what follows, we shall consider topologies corresponding to $\sigma$ being the collections of all finite sets, all compact sets and all bounded sets which, as in the case of linear operators, will be called respectively the topologies of pointwise convergence, compact convergence and bounded convergence.

We conclude the section by extending the uniform boundedness principle to fans.

4.5. Definition. Let $\mathfrak{A}$ be a family of bounded fans from $X$ into $Y$. We shall say that $\mathfrak{A}$ is upper equicontinuous at $x$ if for any neighbourhood $V \subset Y$ about the origin, there is a neighbourhood $U \subset X$ about the origin such that

$$
\mathbb{Q}(x+h) \subset \mathbb{Q}(x)+V, \text { for any } h \in U, \mathbb{Q} \in \mathfrak{A} \text {. }
$$

It is easy to see that $\mathfrak{A}$ is upper equicontinuous at the origin iff for any $V$ there is a $U$ such that $s_{\mathbb{Q}}\left(y^{*}, x\right)<1$, for all $x \in U, y^{*} \in V^{0}, \mathbb{Q} \in \mathfrak{A}$.

If $U$ can be chosen in such a way that also $\mathbb{Q}(x) \subset \mathbb{Q}(x+h)+V$, for any $h \in U, \mathbb{Q} \in \mathfrak{A}$, then we shall say that $\mathfrak{A}$ is equicontinuous at $x$. This is the same as

$$
\left|s_{\mathscr{Q}}\left(y^{*}, x\right)-s_{\mathscr{Q}}\left(y^{*}, x+h\right)\right|<1, \quad \text { for all } h \in U, y^{*} \in V^{0}, \mathbb{Q} \in \mathfrak{A} \text {. }
$$

Finally, if for any given $V$ the same $U$ can be chosen for all $x \in X$, we shall say that $\mathfrak{A}$ is uniformly upper equicontinuous (resp. equicontinuous).

4.6. Proposition. The following four properties are equivalent:

(a) $\mathfrak{A}$ is upper equicontinuous at the origin;

(b) $\mathfrak{A}$ is equicontinuous at the origin;

(c) $\mathfrak{A}$ is uniformly upper equicontinuous;

(d) $\mathfrak{A}$ is uniformly equicontinuous.

Proof. Clearly (d) $\Rightarrow(c) \Rightarrow(b) \Rightarrow$ (a) so that it remains to prove the implication (a) $\Rightarrow$ (d).

If $\mathfrak{A}$ is upper equicontinuous at the origin, then for a given $V \subset Y$, we can choose $U \subset X$ such that $\mathbb{Q}(x) \subset V / 2$ if $x \in U, \mathbb{Q} \in \mathfrak{A}$. We can assume that $U=-U$ so that $x-u \in U$ and $u-x \in U$ simultaneously. In this case, for any $\mathbb{Q} \in \mathfrak{A}$ we have

$$
\mathbb{Q}(x) \subset \overline{\mathbb{Q}(u)+\mathbb{Q}(x-u)} \subset \overline{\mathbb{Q}(u)+V / 2} \subset \mathbb{Q}(u)+V .
$$

The inclusion $\mathbb{Q}(u) \subset \mathbb{Q}(x)+V$ can be verified similarly.

4.7. TheORem. Let $\mathfrak{A}$ be a family of bounded fans from $X$ into $Y$ which is upper equicontinuous at the origin. Then the restrictions on $\mathfrak{A}$ of the topologies of pointwise and compact convergence coincide. 
Proof. Given a neighbourhood $V \subset Y$ about the origin and a compact $M \subset X$, we have to show that for any $\varepsilon>0$ there is a finite set $M^{\prime} \subset X$ such that

$$
\rho_{M, V}(\mathscr{Q}, \mathscr{B}) \leqslant \rho_{M^{\prime}, V}(\mathscr{Q}, \mathscr{B})+\varepsilon, \text { for all } \mathbb{Q}, \mathscr{B} \in \mathfrak{A} \text {. }
$$

Making use of 4.6, we can choose an open $U \subset X$ containing 0 such that $\sup \left\{\left|s_{\mathbb{Q}}\left(y^{*}, x+h\right)-s_{\mathbb{Q}}\left(y^{*}, x\right)\right| \mid y^{*} \in V^{0}, h \in U\right\}<\varepsilon / 2$ for all $x \in X$ and $\mathbb{Q} \in$ Ar. Take a finite collection $M^{\prime}=\left\{x_{1}, \ldots, x_{n}\right\}$ of points of $X$ such that $M \subset$ $\cup\left(x_{i}+U\right)$. Let $M_{i}=\left(x_{i}+U\right) \cap M$. Then for any $\mathbb{Q}, \mathfrak{B} \in \mathfrak{A}$,

$$
\begin{aligned}
\rho_{M, V}(\mathcal{Q}, \mathscr{B}) \leqslant & \max _{i} \sup \left\{\left|s_{\mathscr{Q}}\left(y^{*}, x\right)-s_{\mathscr{B}}\left(y^{*}, x_{i}\right)\right| \mid y^{*} \in V^{0}, x \in M_{i}\right\} \\
& +\max _{i} \sup \left\{\left|s_{\mathscr{Q}}\left(y^{*}, x_{i}\right)-s_{\mathscr{B}}\left(y^{*}, x_{i}\right)\right| \mid y^{*} \in V^{0}\right\} \\
& +\max _{i} \sup \left\{\left|s_{\mathscr{B}}\left(y^{*}, x_{i}\right)-s_{\mathscr{B}}\left(y^{*}, x\right)\right| \mid y^{*} \in V^{0}, x \in M_{i}\right\} \\
\leqslant & \rho_{M^{\prime}, V}(\mathbb{Q}, \mathscr{B})+\varepsilon .
\end{aligned}
$$

4.8. TheOrem. Assume that $X$ is a barrel space and $\mathfrak{A}$ is a pointwise bounded family of elements of $\mathscr{F}(X, Y)$. Then $\mathfrak{A}$ is upper equicontinuous at the origin.

Pointwise boundedness means boundedness in the topology of pointwise convergence, in other words, that for any $x \in X$ the set $\{\cup \mathbb{Q}(x) \mid \mathbb{Q} \in \mathfrak{A}\}$ is bounded in $Y$. Observe that, according to the definition, any family of bounded fans which is upper equicontinuous at the origin is bounded in every $\sigma$-topology, in particular pointwise bounded.

Proof. Fix a neighbourhood $V \subset Y$ about the origin. Since any set $B(x)=$ $\{\cup \mathscr{Q}(x) \mid \mathscr{Q} \in \mathfrak{A}\}$ is bounded, the function

$$
\begin{aligned}
f(x) & =\sup \left\{s_{\mathbb{Q}}\left(y^{*}, x\right) \mid y^{*} \in V^{0}, \mathbb{Q} \in \mathfrak{A}\right\} \\
& =\sup \left\{\left\langle y^{*}, y\right\rangle \mid y \in B(x), y^{*} \in V^{0}\right\}
\end{aligned}
$$

is everywhere finite. On the other hand, this function, being the upper bound of a family of continuous sublinear functions, is convex and 1.s.c. Since $X$ is a barrel space, it follows that $f$ is continuous. Then $U=\left\{x \mid f(x)<\frac{1}{2}\right\}$ is open and contains the origin. But $x \in U$ only if $B(x) \subset \overline{V / 2} \subset V$.

\section{Fans and prefans in Banach spaces.}

5.1. Definition. Let $X$ and $Y$ be Banach spaces. For any prefan $\mathcal{Q}$ from $X$ into $Y$ we define the norm of $\mathbb{Q}$ by

$$
\|\mathbb{Q}\|=\sup \{\|y\| \mid y \in \mathbb{Q}(x),\|x\|<1\},
$$

and the diameter of $\mathbb{Q}$ by

$$
\operatorname{diam} \mathbb{Q}=\sup \left\{\left\|y_{1}-y_{2}\right\| \mid y_{i} \in \mathbb{Q}(x),\|x\|<1\right\} .
$$

The Hausdorff distance between two bounded prefans is

$$
H(\mathscr{Q}, \mathscr{B})=\sup \{H(\mathscr{Q}(x), \mathscr{B}(x)) \mid\|x\|<1\} .
$$


5.2. PROPOSITION. The following formulas hold:

$$
\begin{aligned}
\|\mathscr{Q}\| & =\sup \left\{s\left(y^{*}, x\right) \mid\left\|y^{*}\right\| \leqslant 1,\|x\|<1\right\} ; \\
\operatorname{diam} \mathscr{Q} & =\sup \left\{s\left(y^{*}, x\right)+s\left(-y^{*}, x\right) \mid\left\|y^{*}\right\|<1,\|x\|<1\right\} ; \\
H(\mathscr{Q}, \mathscr{B}) & =\sup \left\{\left|s_{\mathscr{Q}}\left(y^{*}, x\right)-s_{\mathscr{B}}\left(y^{*}, x\right)\right| \mid\left\|y^{*}\right\|<1,\|x\|<1\right\} .
\end{aligned}
$$

Proof. Let us prove the last formula (proofs of the first two are even simpler). We have

$$
\sup _{y \in \mathscr{Q}(x)} \inf _{v \in \mathscr{B}(x)}\|y-v\|=\sup _{y \in \mathbb{P}(x)} \inf _{v \in \mathscr{B}(x)} \sup _{\left\|y^{*}\right\|<1}\left\langle y^{*}, y-v\right\rangle
$$

(applying the Ky Fan theorem)

$$
=\sup \left\{s_{\mathfrak{Q}}\left(y^{*}, x\right)-s_{\mathfrak{B}}\left(y^{*}, x\right) \mid\left\|y^{*}\right\|<1\right\}
$$

so that $H(\mathbb{Q}(x), \mathscr{B}(x))=\sup \left\{\left|s_{\mathbb{Q}}\left(y^{*}, x\right)-s_{\mathscr{B}}\left(y^{*}, x\right)\right| \mid\left\|y^{*}\right\|<1\right\}$.

5.3. Proposition. A prefan $\mathbb{Q}$ is bounded if and only if $\|\mathbb{Q}\|<\infty$.

Proof. This is obvious.

5.4. Proposition.

$$
\begin{aligned}
& \|\mathbb{Q}+\mathscr{B}\| \leqslant\|\mathbb{Q}\|+\|\mathscr{B}\|, \\
& \|\mathscr{B} \circ \mathbb{Q}\| \leqslant\|\mathscr{B}\|\|\mathbb{Q}\| \text {, } \\
& \|\mathbb{Q} \vee \mathscr{B}\| \leqslant \sup \{\|\mathbb{Q}\|,\|\mathscr{B}\|\} \text {, } \\
& \operatorname{diam}(\mathscr{Q}+\mathscr{B}) \leqslant \operatorname{diam} \mathscr{Q}+\operatorname{diam} \mathscr{B}, \\
& \operatorname{diam}(\mathscr{B} \circ \mathbb{Q}) \leqslant \operatorname{diam} \mathscr{B}+\|\mathscr{B}\| \operatorname{diam} \mathbb{Q} \text {. }
\end{aligned}
$$

ProOF. All formulas are easily verified by direct calculation.

5.5. Proposition. Let $\mathcal{Q}$ and $\mathscr{B}$ be bounded fans. Then

$$
\|\mathbb{Q}\|=\left\|\mathbb{Q}^{*}\right\|, \quad H(\mathbb{Q}, \mathscr{B})=H\left(\mathbb{Q}^{*}, \mathfrak{B}^{*}\right),
$$

and if $Q$ is odd, then

$$
\operatorname{diam} \mathbb{Q}=\operatorname{diam} \mathbb{Q}^{*}
$$

Proof. This follows from 5.2 since $Q$ and $Q^{*}$ have the same support function.

5.6. Proposition. Any o-topology in $\mathscr{F}(X, Y)$ associated with the norm topology of $Y$ is defined by pseudometrics

$$
d_{M}(\mathscr{Q}, \mathscr{B})=\sup \{H(\mathscr{Q}(x), \mathscr{B}(x)) \mid x \in M\} \quad(M \in \sigma) .
$$

In particular, $H(\mathscr{Q}, \mathscr{B})$ is a metric corresponding to the topology of bounded convergence. With this metric, the space $\mathscr{F}(X, Y)$ is complete.

Proof. All except completeness follows from the fact that, in a Banach space, balls about the origin form a base of neighbourhoods. Completeness results from the corresponding property of the space of convex closed bounded sets with the Hausdorff metrics. 
5.7. Proposition (Banach-Steinhaus Theorem). A family $\mathfrak{A}$ of bounded fans from $X$ into $Y$ is equicontinuous if and only if it is bounded in the metric topology, i.e. if $\|\mathbb{Q}\| \leqslant k<\infty$ for all $\mathbb{Q} \in \mathfrak{X}$. In particular, if a sequence $\left\{\mathbb{Q}_{n}\right\}$ of bounded fans is such that, for any $x \in X, \mathbb{Q}_{n}(x)$ Hausdorff converges to a certain $\mathbb{Q}(x)$, then $\mathbb{Q}$ is also a bounded fan, $\mathbb{Q}_{n}$ converges to $\mathbb{Q}$ in the topology of compact convergence and the norms of $\mathbb{Q}_{n}$ are uniformly bounded.

Proof. The first part is obvious; the second follows from Theorem 4.7.

5.8. REMARK. The assumptions of the second part of the last proposition are satisfied if $\left\{\mathscr{Q}_{n}\right\}$ is a decreasing sequence of bounded fans and there is a prefan $\mathbb{Q}_{0}$ with nonempty and norm compact values such that $\mathbb{Q}_{n}(x) \subset \mathbb{Q}_{0}(x)+\delta_{n}\|x\| B_{X}$ for all $x$ and $n$, where $\delta_{n} \rightarrow 0$.

5.9. Definition. Let $\mathcal{Q}$ be a prefan from $X$ into $Y$ and $K \subset X$ a nonempty cone. Let, furthermore, $\mathscr{D}$ denote the collection of all sequences $\left\{D_{n}\right\}$ of weak ${ }^{*}$ closed subsets of $B_{Y^{*}}$ such that $\lim _{n \rightarrow \infty} \inf \left\{\left\|y^{*}\right\| \mid y^{*} \in D_{n}\right\}=1$.

We define the Banach constant of $Q$ on $K$ by

$$
C(\mathscr{Q}, K)=-\sup _{\left\|y^{*}\right\|=1} \inf _{x \in B_{K}} s\left(y^{*}, x\right)
$$

and the slope of $\mathbb{Q}$ on $K$ by

$$
\operatorname{sl}(\mathbb{Q}, K)=-\sup _{\left\{D_{n}\right\} \in \mathbb{D}} \inf _{x \in B_{K}} \lim _{n \rightarrow \infty} \sup _{y^{*} \in D_{n}} s\left(y^{*}, x\right),
$$

where $B_{K}=B_{X} \cap K$. We write also $C(\mathbb{Q})=C(\mathbb{Q}, X)$, sl(Q) $=\operatorname{sl}(\mathscr{Q}, X)$.

5.10. Definition. Let $\mathscr{B}$ be a homogeneous set-valued mapping from $Y^{*}$ into $X^{*}$ and $P \subset X^{*}$ a cone. We set

$$
\begin{aligned}
& C^{*}(\mathscr{B}, P)=\inf \left\{\left\|x^{*}+u^{*}\right\| \mid u^{*} \in P, x^{*} \in \mathscr{B}\left(y^{*}\right),\left\|y^{*}\right\|=1\right\}, \\
& \mathrm{sl}^{*}(\mathscr{B}, P)=\inf _{\left\{D_{n}\right\} \in \mathscr{D}} \inf \left\{\left\|x^{*}+u^{*}\right\| \mid u^{*} \in P, x^{*} \in \bigcap_{n} W\left(\mathscr{B}, D_{n}\right)\right\}
\end{aligned}
$$

where $W(\mathscr{B}, D)=\overline{\text { conv }} \cap_{y^{*} \in D} \mathscr{B}\left(y^{*}\right)$ (the weak* closure).

The following simple proposition summarizes some elementary properties of the four constants. We set

$$
\|\mathbb{Q}\|_{K}=\sup \left\{s\left(y^{*}, x\right)\|\| y^{*} \|<1, x \in B_{K}\right\} .
$$

\subsection{Proposition.}

$$
C(\mathscr{Q}, K) \geqslant C\left(\mathbb{Q}^{\prime}, K^{\prime}\right), \quad \operatorname{sl}(\mathbb{Q}, K) \geqslant \operatorname{sl}\left(\mathbb{Q}^{\prime}, K^{\prime}\right)
$$

if $K^{\prime} \subset K$ and $\mathscr{Q}(x) \subset \mathbb{Q}^{\prime}(x)$ for all $x$;

$$
C^{*}(\mathscr{B}, P) \geqslant C^{*}\left(\mathscr{B}^{\prime}, P^{\prime}\right), \quad \mathrm{sl}^{*}(\mathscr{B}, P) \geqslant \mathrm{sl}^{*}\left(\mathscr{B}^{\prime}, P^{\prime}\right)
$$

if $P \subset P^{\prime}$ and $\mathscr{B}\left(y^{*}\right) \subset \mathscr{B}^{\prime}\left(y^{*}\right)$ for all $y^{*}$;

$$
\begin{aligned}
C\left(\mathscr{Q}+\mathbb{Q}^{\prime}, K\right) & \geqslant C(\mathscr{Q}, K)-\left\|\mathbb{Q}^{\prime}\right\|_{K}, \\
\mathrm{sl}\left(\mathscr{Q}+\mathbb{Q}^{\prime}, K\right) & \geqslant \operatorname{sl}(\mathscr{Q}, K)-\left\|\mathbb{Q}^{\prime}\right\|_{K}, \\
C^{*}\left(\mathscr{B}+\mathscr{B}^{\prime}, P\right) & >C^{*}(\mathscr{B}, P)-\left\|\mathscr{B}^{\prime}\right\|, \\
\mathrm{sl}^{*}\left(\mathscr{B}+\mathscr{B}^{\prime}, P\right) & >\mathrm{sl}^{*}(\mathscr{B}, P)-\left\|\mathscr{B}^{\prime}\right\| .
\end{aligned}
$$


5.12. TheOrem. Let $Q$ be a bounded fan from $X$ into $Y$ and $K \subset X$ a nonempty convex cone. Then

$$
C(\mathbb{Q}, K)=C^{*}\left(\mathbb{Q}^{*}, K^{0}\right), \quad \operatorname{sl}(\mathbb{Q}, K)=\mathrm{sl}^{*}\left(\mathscr{Q} *, K^{0}\right),
$$

where $K^{0}=\left\{x^{*} \mid\left\langle x^{*}, x\right\rangle \leqslant 0, \forall x \in K\right\}$ is the polar of $K$.

Proof. What we need to show is that for any nonempty weak* compact convex set $Q$ and nonempty convex cone $K \subset X$,

$$
-\inf \left\{\delta^{*}(x, Q) \mid\|x\| \leqslant 1, x \in K\right\}=\inf \left\{\left\|x^{*}+u^{*}\right\| \mid u^{*} \in K^{0}, x^{*} \in Q\right\} .
$$

The desired formulas will follow from here if we take $Q=Q^{*}\left(y^{*}\right)$ and $Q=$ $\cap_{n} W\left(Q^{*}, D_{n}\right)$, respectively.

We have

$$
\begin{aligned}
a & =\inf \left\{\left\|x^{*}+u^{*}\right\| \mid u^{*} \in K^{0}, x^{*} \in Q\right\} \\
& =\inf _{x^{*} \in Q} \inf _{u^{*} \in K^{0}} \sup _{\|x\|<1}\left\langle x^{*}+u^{*}, x\right\rangle \\
& =\lim _{m \rightarrow \infty} \inf _{x^{*} \in Q} \inf _{\substack{u^{*} \in K^{0} \\
\left\|u^{*}\right\|<m}} \sup _{\|x\|<1}\left\langle x^{*}+u^{*}, x\right\rangle \\
& =\lim _{m \rightarrow \infty} \sup _{\|x\|<1} \inf _{x^{*} \in Q} \inf _{\substack{u^{*} \in K^{0} \\
\left\|u^{*}\right\|<m}}\left\langle x^{*}+u^{*}, x\right\rangle \\
& =\lim _{m \rightarrow \infty} \sup _{\|x\|<1}\left(-\delta^{*}(-x, Q)-m \rho\left(-x, K^{\infty}\right)\right) \\
& =-\lim _{m \rightarrow \infty} \inf _{\|x\|<1}\left(\delta^{*}(x, Q)+m \rho(x, K)\right) \\
& \geqslant-\inf \left\{\delta^{*}(x, Q) \mid\|x\|<1, x \in K\right\} .
\end{aligned}
$$

Here $K^{00}$ is the bipolar of $K$ coinciding with $\bar{K}$ since $K$ is a convex cone.

To prove the opposite inequality, let us find a sequence $\left\{x_{m}\right\} \in X$ such that $\left\|x_{m}\right\|<1$ and

$$
\delta^{*}\left(x_{m}, Q\right)+m \rho\left(x_{m}, K\right) \leqslant \inf \left\{\delta^{*}(x, Q)+m \rho(x, K) \mid\|x\|<1\right\}+1 / m .
$$

Then (since $a<\infty$ if $Q$ and $K$ are nonempty)

$$
0 \leqslant m \rho\left(x_{m}, K\right)<a-\delta^{*}\left(x_{m}, Q\right)<a+\sup \left\{\left\|x^{*}\right\| \mid x^{*} \in Q\right\}
$$

which implies that $\rho\left(x_{m}, K\right) \rightarrow 0$. Let $x_{m}^{\prime} \in K$ be such that $\left\|x_{m}-x_{m}^{\prime}\right\| \rightarrow 0$. Then $\delta^{*}\left(x_{m}, Q\right)-\delta^{*}\left(x_{m}^{\prime}, Q\right) \leqslant \delta^{*}\left(x_{m}-x_{m}^{\prime}, Q\right) \rightarrow 0$ and

$$
\begin{aligned}
\inf \left\{\delta^{*}(x, Q) \mid\|x\| \leqslant 1, x\right. & \in K\}<\liminf _{m \rightarrow \infty} \delta^{*}\left(x_{m}^{\prime}, Q\right)=\liminf _{m \rightarrow \infty} \delta^{*}\left(x_{m}, Q\right) \\
& \leqslant \lim _{m \rightarrow \infty} \inf \left\{\delta^{*}(x, Q)+m \rho(x, K) \mid\|x\|<1\right\}=-a .
\end{aligned}
$$

5.13. Definition. Let

$$
\Delta\left(Y^{*}\right)=\inf _{\left\{D_{n}\right\} \in \mathscr{D}} \limsup _{n \rightarrow \infty}\left(\operatorname{diam} D_{n}\right) .
$$


5.14. THEOREM. If $Q$ is a prefan from $X$ into $Y$ and $K \subset X$ a cone, then

$$
C(\mathbb{Q}, K) \geqslant \operatorname{sl}(\mathbb{Q}, K) \text {; }
$$

if, in addition, $\mathcal{Q}$ is bounded, then

$$
\operatorname{sl}(\mathbb{Q}, K)>C(\mathbb{Q}, K)-\Delta\left(Y^{*}\right)\|\mathscr{Q}\|_{K}
$$

If $\mathscr{B}$ is a homogeneous set-valued mapping from $Y^{*}$ into $X^{*}$ and $P \subset X^{*}$ is a cone, then

$$
C^{*}(\Re, P) \geqslant \mathrm{sl}^{*}(\Re, P)
$$

if, in addition, $\mathscr{B}$ is a fan adjoint to a bounded fan from $X$ into $Y$, then

$$
\mathrm{sl}^{*}(\mathscr{B}, P)>C^{*}(\mathscr{B}, P)-\operatorname{diam} \mathscr{B} \text {. }
$$

Proof. To prove the first and third inequalities, one should consider stationary sequences $\left\{D_{n}\right\}$ of singletons (i.e. $D_{n}=\left\{y^{*}\right\}$, where $y^{*}$ is the same for all $n$ ).

To obtain the second inequality, we first observe that for $y^{*}, v^{*} \in Y^{*}, x \in B_{K}$,

$$
s\left(y^{*}, x\right) \leqslant s\left(v^{*}, x\right)+s\left(y^{*}-v^{*}, x\right) \leqslant s\left(v^{*}, x\right)+\left\|y^{*}-v^{*}\right\|\|\mathbb{Q}\|_{K} .
$$

If $\left\{D_{n}\right\} \in \mathscr{D}$ and $v^{*} \in \cap D_{n}$, then $\left\|v^{*}\right\|=1$ and

$$
\begin{aligned}
-\operatorname{sl}(\mathscr{Q}, K) & \leqslant \inf _{x \in B_{K}} \lim _{n \rightarrow \infty}\left(s\left(v^{*}, x\right)+\left(\sup _{y^{*} \in D_{n}}\left\|y^{*}-v^{*}\right\|\right)\|\mathbb{Q}\|_{K}\right) \\
& \leqslant-C(\mathbb{Q}, K)+\Delta\left(Y^{*}\right)\|\mathbb{Q}\|_{K} .
\end{aligned}
$$

It remains to prove the last inequality. If $x^{*} \in W(\Re, D)$, then $x^{*}$ is a weak limit of a net $\left\{x_{\lambda}^{*}\right\}$ such that $x_{\lambda}^{*}=\sum_{i=1}^{n(\lambda)} \alpha_{i \lambda} x_{i \lambda}^{*}, x_{i \lambda}^{*} \in \mathscr{B}\left(y_{i}^{*}\right), y_{i \lambda}^{*} \in D, \alpha_{i \lambda} \geqslant 0$, $i=1, \ldots, n(\lambda), \sum_{i=1}^{n(\lambda)} \alpha_{i \lambda}=1$.

Let $y_{\lambda}^{*}=\sum_{i=1}^{n(\lambda)} \alpha_{i \lambda} y_{i}^{*}$. If $D$ is a weak ${ }^{*}$ closed subset of the unit ball and if $D$ is convex, then, taking if necessary a subnet, we may assume that $y_{\lambda}^{*}$ converge in the weak* topology to some $y^{*} \in D$.

Since $\mathscr{B}$ is the adjoint to a fan from $X$ into $Y, 1.10$ implies the existence of $w_{\lambda}^{*} \in \mathscr{B}\left(y_{\lambda}^{*}\right)$ converging in the weak* topology to some $w^{*} \in \mathscr{B}\left(y^{*}\right)$. On the other hand, every $w_{\lambda}^{*}$ can be represented as

$$
w_{\lambda}^{*}=\sum_{i=1}^{n(\lambda)} \alpha_{i \lambda} w_{i \lambda}^{*}, \quad w_{i \lambda}^{*} \in \mathscr{B}\left(y_{i \lambda}^{*}\right)
$$

(the closure operation in (1.1.4) is needless here thanks to 1.13). We have, therefore,

$$
x_{\lambda}^{*}=w_{\lambda}^{*}+\sum_{i=1}^{n(\lambda)} \alpha_{i \lambda}\left(x_{i \lambda}^{*}-w_{i \lambda}^{*}\right)=w_{\lambda}^{*}+z_{\lambda}^{*}
$$

and the net $\left\{z_{\lambda}^{*}\right\}$ converges to $z^{*}=x^{*}-w^{*}$ so that

$$
\left\|z^{*}\right\| \leqslant \lim \inf \left\|z_{\lambda}^{*}\right\| \leqslant \lim \inf \sum_{i=1}^{n(\lambda)} \alpha_{i \lambda}\left\|x_{i}^{*}-w_{i}^{*}\right\|<\operatorname{diam} \mathscr{B} .
$$

(Recall that $D \subset B_{Y^{*}}$ ). 
Let $r(D)=\inf \left\{\left\|y^{*}\right\| \mid y^{*} \in D\right\}$. Then

$$
\begin{aligned}
\inf \left\{\| x^{*}+\right. & \left.u^{*} \| \mid u^{*} \in P, x^{*} \in W(\mathscr{B}, D)\right\} \\
\geqslant & \inf \left\{\left\|w^{*}+z^{*}+u^{*}\right\| \mid u^{*} \in P, w^{*} \in \mathscr{B}\left(y^{*}\right), y^{*} \in D,\left\|z^{*}\right\| \leqslant \operatorname{diam} \mathscr{B}\right\} \\
> & \inf \left\{\left\|w^{*}+u^{*}\right\| \mid u^{*} \in P, w^{*} \in \mathscr{B}\left(y^{*}\right), y^{*} \in D\right\}-\operatorname{diam} \mathscr{B} \\
> & \inf (D)<k<1 \\
& -\operatorname{diam}\left\{\left\|\left(w^{*} / k\right)+u^{*}\right\| \mid u^{*} \in P, w^{*} \in k \mathscr{B}\left(y^{*}\right),\left\|y^{*}\right\|=1\right\} \\
= & r(D) C^{*}(\mathscr{B}, P)-\operatorname{diam} \mathscr{B} .
\end{aligned}
$$

It remains to take into account that $r\left(D_{n}\right) \rightarrow 1$ if $\left\{D_{n}\right\} \in \mathscr{D}$.

5.15. THEOREM. If $Q$ is a bounded prefan from $X$ into $Y$, then the equality $C(\mathbb{Q}, K)=\operatorname{sl}(\mathscr{Q}, K)$ holds if either

(a) $\|\cdot\|$ is a locally uniformly convex norm in $Y^{*}$, or

(b) the values of $Q$ are norm compact and $\|\cdot\|$ is strictly convex in $Y^{*}$.

The equality $C^{*}(\Re, P)=\mathrm{sl}^{*}(\mathscr{B}, P)$ holds if

(c) $\mathscr{B}$ is a linear operator adjoint to a bounded linear operator from $X$ into $Y$.

In particular, if $\mathbb{Q}$ is a bounded fan from $X$ into $Y, \mathscr{B}=\mathbb{Q}^{*}, K$ is convex and $P=K^{0}$, then all four quantities coincide, provided one of the conditions (a)-(c) is satisfied.

Proof. If the norm in $Y^{*}$ is locally uniformly convex, then any sequence $\left\{D_{n}\right\}$ norm converges to a point $y^{*}$ of the unit sphere in the sense that

$$
\sup \left\{\left\|y^{*}-v^{*}\right\| \mid v^{*} \in D_{n}\right\} \rightarrow 0 \text {. }
$$

Since the support function of a bounded fan is norm continuous in each variable, it follows that

$$
\lim _{n \rightarrow \infty} \sup _{v^{*} \in D_{n}} s\left(v^{*}, x\right)=s\left(y^{*}, x\right)
$$

which implies the desired equality.

If the norm in $Y^{*}$ is strictly convex, then $\cap D_{n}$ is a singleton whenever $\left\{D_{n}\right\} \in \mathscr{D}$. If now $\mathcal{Q}$ is norm-compact-valued, then $s\left(y^{*}, x\right)$ is weak continuous in $y^{*}$ on every norm bounded subset of $Y^{*}$ and we come again to the limit relation (1).

The two last statements of the theorem follow from 5.14 and 5.12 respectively.

5.16. REMARK. The four constants introduced in 5.9, 5.10 will play a very important role in the next chapter (see also the next section) for they decisively enter the "modulus of surjectivity" of nonsmooth mappings (see \$11). As a matter of fact, the estimations of the moduli originally include only slopes $\operatorname{sl}(\mathbb{Q}, K)$ and $\mathrm{sl}^{*}(\mathscr{B}, P)$. Banach constants $C(\mathscr{Q}, K)$ and $C^{*}(\mathscr{B}, P)$ appear to be useful only as long as they, in turn, provide estimations for slopes which are less convenient to handle than Banach constants.

But slopes have one more disadvantage which seems to be even more serious. Banach constants are stable under perturbations of the norm: for an equivalent norm in $Y^{*}$ close to $\|\cdot\|$, Banach constants corresponding to this norm are close to 
those corresponding to $\|\cdot\|$. On the contrary, $\operatorname{sl}(Q, K)$ and $\operatorname{sl}^{*}(\mathscr{B}, P)$ essentially depend on geometry of the unit ball, and small perturbations of the norm can cause noticeable variations of their values.

This suggests the following definition.

5.17. Definition. We set

$$
\begin{aligned}
\operatorname{Sl}(\mathscr{Q}, K) & =\underset{|\cdot| \rightarrow\|\cdot\|}{\lim \sup } \operatorname{sl}(\mathscr{Q}, K,|\cdot|), \\
\mathrm{Sl}^{*}(\mathscr{B}, P) & =\underset{|\cdot| \rightarrow\|\cdot\|}{\lim \sup } \operatorname{sl}^{*}(\mathscr{B}, P,|\cdot|),
\end{aligned}
$$

where $\operatorname{sl}(\mathscr{Q}, K,|\cdot|)$ and $\operatorname{sl}^{*}(\mathscr{B}, P,|\cdot|)$ are defined as in $5.9,5.10$ but by way of the $|\cdot|$-norm in $Y^{*}$ and the symbol $|\cdot| \rightarrow \mid \cdot \|$ obviously means that equivalent norms | $\cdot$ | converge to $\|\cdot\|$ (uniformly on bounded subsets of $Y^{*}$ ).

One can easily verify that all the properties of $\mathrm{sl}$ and $\mathrm{sl}^{*}$ discussed above can be extended to $\mathrm{Sl}$ and $\mathrm{Sl}^{*}$. Moreover, some of them can even be strengthened.

5.18. Proposition (CF. 5.14). If $\mathbb{Q}$ is a bounded prefan from $X$ into $Y$, then

$$
\operatorname{Sl}(\mathscr{Q}, K) \geqslant C(\mathscr{Q}, K)-\delta\left(Y^{*}\right)\|\mathscr{Q}\|_{K},
$$

where $\delta\left(Y^{*}\right)=\lim \inf _{|\cdot| \rightarrow\|\cdot\|} \Delta\left(Y^{*},|\cdot|\right)$ and $\Delta\left(Y^{*},|\cdot|\right)$ is defined as in 5.13 with $|\cdot|$ instead of $\|\cdot\|$.

Proof. The only circumstance that should be taken into account in addition to 5.14 is that the Banach constant depends continuously on equivalent norms.

5.19. TheOREM. Let $\mathbb{Q}$ be a bounded prefan from $X$ into $Y$. Then the equality $C(\mathbb{Q}, K)=\mathrm{Sl}(\mathbb{Q}, K)$ holds if either

(a) there is an equivalent locally uniformly convex norm in $Y^{*}$, or

(b) $\mathbb{Q}$ is norm-compact-valued and there is an equivalent strictly convex norm in $Y^{*}$.

Proof. The only additional consideration to be incorporated is that in either case there is a sequence of locally uniformly or strictly convex norms converging to $\|\cdot\|$.

5.20. RemarK. Observe that, in general, the sequence of equivalent norms that realize $\mathrm{Sl}(\mathcal{Q}, K)$ or $\mathrm{Sl}^{*}(\mathscr{B}, P)$ depends on $\mathbb{Q}, K, \mathfrak{B}, P$. On the contrary, under the assumptions (a), (b) of the theorem, the same sequence can be chosen for all $\mathcal{Q}, K$, $\mathscr{B}, P$.

\section{Open mapping theorem.}

6.1. A mapping is said to be open if it transforms open sets into open sets. The classical Banach open mapping theorem says that any bounded linear operator from a Banach space onto another Banach space is an open mapping. In this form, this principle cannot be extended to fans. However, it admits an equivalent formulation which turns out to be extendable.

Let $A: X \rightarrow Y$ be a linear bounded operator which sends $X$ onto $Y$. Simple category arguments (with which the proof of the Banach open mapping theorem usually begins) imply that the closure of $A\left(B_{X}\right)$ contains a ball about the origin 
with radius $r>0$. This is the same as

$$
A^{*} y^{*}=\sup \left\{\left\langle y^{*}, A x\right\rangle \mid\|x\| \leqslant 1\right\}>r\left\|y^{*}\right\|
$$

for all $y^{*}$, which is equivalent to $C^{*}\left(A^{*}\right)$ (and hence $C(A)$ due to 5.12) being positive.

Thus the Banach open mapping theorem can be reformulated as follows: if $C(A)>0$, then $A$ is an open mapping. This is just the equivalent form of the Banach open mapping theorem which can be extended to fans.

First we consider the finite-dimensional case.

6.2. TheORem. Assume that $\operatorname{dim} Y<\infty$. Then for any $Q \in \mathscr{F}(X, Y)$ and for any closed convex cone $K \subset X$,

$$
C(\mathbb{Q}, K) U_{Y} \subset \mathbb{Q}\left(B_{X} \cap K\right)
$$

Proof. Fix $\mathbb{Q}$ and $K$. If $C(\mathbb{Q}, K)=0$, the theorem is obvious. So let us assume that $C(Q, K)=r>0$.

The theorem will be proved if for any $\varepsilon>0$ we shall find a finite-dimensional subspace $X^{\prime} \subset X$ and continuous mappings $f: X^{\prime} \rightarrow Y, g: Y \rightarrow Y^{*}, h: Y^{*} \rightarrow X^{\prime}$ such that

$$
\begin{aligned}
& f(x) \in \mathbb{Q}(x), \quad \text { for all } x \in X^{\prime} ; \\
& \|g(y)\|=\|y\|, \quad\langle g(y), y\rangle>0, \quad \text { for all } y \in Y ; \\
& \left\|h\left(y^{*}\right)\right\|=\left\|y^{*}\right\|, \quad h\left(y^{*}\right) \in K, \quad \text { for all } y^{*} \in Y^{*} ; \\
& \left\langle y^{*},(f \circ h)\left(y^{*}\right)\right\rangle<-(r-\varepsilon)\left\|y^{*}\right\|^{2}, \quad \text { for all } y^{*} \in Y^{*} .
\end{aligned}
$$

Indeed, assume that such $X^{\prime}, f, g, h$ exist. Let $p=f \circ h \circ g$, and for any $v \in Y$ let

$$
p_{v}(y)=\left\{\begin{array}{l}
p(y)-v+y, \text { if }\|p(y)-v+y\| \leqslant 1, \\
\frac{p(y)-v+y}{\|p(y)-v+y\|}, \quad \text { if }\|p(y)-v+y\|>1 .
\end{array}\right.
$$

Then the restriction of $p_{v}$ to $B_{Y}$ is a continuous mapping from $B_{Y}$ into itself so that, according to the Brouwer fixed point theorem, there is $y \in B_{Y}$ such that $p_{v}(y)=y$.

If $\lambda=\|p(y)-v+y\|>1$, then $\|y\|=1$ and $p(y)-v+y=\lambda y$ or

$$
\langle g(y), v\rangle=\langle g(y), p(y)\rangle-(\lambda-1)\langle g(y), y\rangle\langle\langle g(y), p(y)\rangle
$$

because $\lambda>1,\langle g(y), y\rangle>0$.

We have, setting $g(y)=y^{*}$,

$$
\langle g(y), p(y)\rangle=\left\langle y^{*},(f \circ h)\left(y^{*}\right)\right\rangle\left\langle-(r-\varepsilon)\left\|y^{*}\right\|^{2}=-(r-\varepsilon)\|y\|^{2}\right.
$$

so that

$$
-\|v\|\|y\|=-\|v\|\|g(y)\|\left\langle\langle g ( y ) , v \rangle \left\langle-(r-\varepsilon)\|y\|^{2}\right.\right.
$$

or

$$
\|v\|>(r-\varepsilon)\|y\|=r-\varepsilon .
$$

It follows that, whenever $\|v\|<r-\varepsilon$, there is $y \in B_{Y}$ such that $p_{v}(y)=p(y)-$ $v+y=y$ or $p(y)=v$. On the other hand, $p(y)=f(x)$, where $x=(h \circ g)(y)$, so 


$$
\begin{aligned}
& \text { that } x \in K \text { and }\|x\|=\|h(g(y))\|=\|g(y)\|=\|y\|<1 \text {. Thus } \\
& (r-\varepsilon) U_{Y} \subset f\left(B_{X} \cap K\right) \subset \mathbb{Q}\left(B_{X} \cap K\right)
\end{aligned}
$$

and, since $\varepsilon>0$ is arbitrary, the desired result follows.

Thus we need to prove the existence of $X^{\prime}, f, g, h$ with necessary properties. According to $1.12, Q$ is a continuous set-valued mapping. Thus, thanks to the continuous selection theorem of Michael [40], the set of continuous selections of $Q$ is nonempty. Let $f$ be one of them.

Let furthermore $e(y)$ be a smooth norm on $Y$, say euclidean, and let $q(y)$ be the derivative of $e^{2}(y)$ at $y$. Then

$$
g(y)=\left\{\begin{array}{l}
0, \quad \text { if } y=0, \\
\|y\|\|q(y)\|^{-1} q(y), \quad \text { if } y \neq 0
\end{array}\right.
$$

meets the necessary requirements (see [44] for the properties of gauge functions and their derivatives).

It remains to construct $h$ and $X^{\prime}$. Fix $\varepsilon>0$. Then for any $y^{*},\left\|y^{*}\right\|=1$, there is $x \in B_{X} \cap K$ such that $s\left(y^{*}, x\right) \leqslant-r+(\varepsilon / 4)$. For any fixed $x$ the function $s(\cdot, x)$, being everywhere defined and convex on a finite-dimensional space, is continuous. Therefore, we can find a finite collection of elements of $B_{X} \cap K$ such that for any $y^{*},\left\|y^{*}\right\|=1$, the inequality $s\left(y^{*}, x\right)<-(r-(\varepsilon / 2))$ holds for a certain element $x$ of the collection. Let $X^{\prime}$ be the subspace spanned by such elements $x$ and $K^{\prime}=X^{\prime} \cap K$.

Therefore if $\mathbb{Q}^{\prime}$ is the restriction of $\mathscr{Q}$ to $X^{\prime}$, then $C\left(\mathbb{Q}^{\prime}, K^{\prime}\right)>r-(\varepsilon / 2)$.

Now let $\|\cdot\|^{\prime}$ be a strictly convex norm on $X^{\prime}$ such that $\|x\|^{\prime}<\|x\|$. Consider the fan $\mathscr{B}_{\varepsilon}(x)=(\varepsilon / 2)\|x\|^{\prime} B_{Y}$ and let $\mathbb{Q}_{\varepsilon}=\mathbb{Q}^{\prime}+\mathscr{B}_{e}$. Then $\left\|\mathscr{B}_{\varepsilon}\right\|<\varepsilon / 2$ and, according to 6.2 ,

$$
C\left(\mathbb{Q}_{e}, K^{\prime}\right) \geqslant C\left(\mathbb{Q}^{\prime}, K^{\prime}\right)-\left\|\Re_{e}\right\| \geqslant r-\varepsilon>0
$$

if $\varepsilon$ is sufficiently small.

It follows that, whenever $\left\|y^{*}\right\|=1$,

$$
\inf \left\{s_{e}\left(y^{*}, x\right) \mid\|x\|<1, x \in K^{\prime}\right\} \leqslant-r+\varepsilon<0,
$$

where $s_{e}$ is the support function of $\mathcal{Q}_{e}$. In view of the compactness of $B_{X} \cap K^{\prime}$ and positive homogeneity of $s_{e}\left(y^{*}, \cdot\right)$, this infimum is attained at some $x$ with $\|x\|=1$. This $x$ is uniquely defined by $y^{*}$ because $\|\cdot\|^{\prime}$ is a strictly convex norm. If we set $x=h\left(y^{*}\right)$, then the function $h$ appears to be defined on the unit sphere of $Y^{*}$ and routine arguments show that it is continuous. We can extend $h$ to all of $Y^{*}$ by setting

$$
h\left(y^{*}\right)=\left\{\begin{array}{l}
0, \quad \text { if } y^{*}=0, \\
\left\|y^{*}\right\| h\left(y^{*} /\left\|y^{*}\right\|\right), \quad \text { if } y^{*} \neq 0 .
\end{array}\right.
$$

Then $s_{e}\left(y^{*}, h\left(y^{*}\right)\right)=\left\|y^{*}\right\|^{2} s_{e}\left(y^{*} /\left\|y^{*}\right\|, h\left(y^{*} /\left\|y^{*}\right\|\right)\right)<-(r-\varepsilon)\left\|y^{*}\right\|^{2}$ so that whenever $y \in \mathbb{Q}\left(h\left(y^{*}\right)\right)$, in particular, if $y=f(x)$, we have

$$
\left\langle y^{*}, y\right\rangle\left\langle s_{\varepsilon}\left(y^{*}, h\left(y^{*}\right)\right)<-(r-\varepsilon)\left\|y^{*}\right\|^{2} .\right.
$$

This completes the proof. 
6.3. Corollary. Let $\operatorname{dim} Y<\infty$, let $\mathbb{Q}$ be a bounded fan from $X$ into $Y$, and let $K \subset X$ be a closed convex cone. Then whenever $f(x)$ is a continuous selection of $\mathbb{Q}$, we have

$$
C(\mathscr{Q}, K) U_{Y} \subset f\left(B_{X} \cap K\right) .
$$

6.4. Remark. Observe that if $\mathcal{Q}$ is a single-valued fan, i.e. a linear operator, the theorem just proved is a trivial fact of linear algebra which needs no topology to be proved. We can replace topological assumptions in the theorem by some algebraic requirements, say, that $K$ and the values of $Q$ be polyhedral. But $I$ have failed to find an algebraic proof of the theorem even under such purely algebraic assumptions. It seems that the "linear algebra" of fans is heavily bound up with topology. We present below another result of this kind extending the (again elementary) proposition that a linear operator which maps a finite-dimensional space onto itself is one-to-one.

6.5. Theorem. Let $\operatorname{dim} X=\operatorname{dim} Y=n<\infty$. Then for any bounded fan from $X$ into $Y, C(\mathbb{Q})=C\left(\mathbb{Q}^{*}\right)$.

Proof. Consider the mapping $h$ as in 6.3. It sends the unit sphere of $Y^{*}$ into the unit sphere of $X$. (We do not assume either $X$ or $Y$ euclidean so that the spheres in $X$ and $Y^{*}$ are boundaries of certain bounded centrally symmetric convex bodies.) It is well defined if $C(\mathbb{Q})=r>0$, and $s\left(y^{*}, h\left(y^{*}\right)\right)<-(r-\varepsilon)$ if $\left\|y^{*}\right\|=1$. It follows that $\left\langle y^{*}, y\right\rangle \leqslant-(r-\varepsilon)$ if $y \in \mathbb{Q}\left(h\left(y^{*}\right)\right),\left\|y^{*}\right\|=1$ so that $\mathbb{Q}\left(h\left(y^{*}\right)\right) \cap$ $Q\left(h\left(-y^{*}\right)\right)=\varnothing$ and hence $h\left(y^{*}\right)$ and $h\left(-y^{*}\right)$ cannot be positively proportional. According to the Borsuk antipodal theorem (see [32] for instance) $h$ maps the unit sphere of $Y^{*}$ onto the unit sphere of $X$.

Now let $\|x\|=1$, and let $y^{*}$ be such that $\left\|y^{*}\right\|=1, x=h\left(y^{*}\right)$. We have

$$
\inf \left\{s\left(z^{*}, x\right) \mid\left\|z^{*}\right\| \leqslant 1\right\} \leqslant s\left(y^{*}, x\right)<-(r-\varepsilon)
$$

so that

$$
C\left(Q^{*}\right)=-\sup _{\|x\|=1} \inf _{\left\|y^{*}\right\|<1} s\left(y^{*}, x\right)>r-\varepsilon
$$

which, since $\varepsilon$ is arbitrary, gives $C\left(Q^{*}\right)>C(\mathscr{Q})$. This inequality obviously holds if $C(\mathbb{Q})=0$ so that it is always true. Changing the roles of $\mathscr{Q}$ and $\mathbb{Q}^{*}$, we obtain the opposite inequality.

6.6. REMARK. None of the results above is valid for prefans. A simple counterexample is the following. Let $f$ be a mapping from $R^{2}$ into $R^{3}$ which sends the unit circle onto two orthogonal equators on the unit sphere. Then $C(f)=\sqrt{2} / 2$ but obviously $f\left(R^{2}\right) \neq R^{3}$. However, as we shall see, prefans are useful for local surjection theorems for nonsmooth mappings.

Now we turn to the general case.

6.7. TheOREM. Let $Q$ be a bounded fan from $X$ into $Y$ and $K$ a closed cone. Then

$$
\operatorname{Sl}(\mathbb{Q},-K) U_{Y} \subset \mathbb{Q}\left(B_{X} \cap K\right) .
$$

Proof. Fix $\varepsilon>0$, and let $|\cdot|$ be an equivalent norm in $Y^{*}$ which is sufficiently close to $\|\cdot\|$ and such that $\operatorname{Sl}(\mathscr{Q},-K)<\operatorname{sl}(\mathbb{Q},-K,|\cdot|)+\varepsilon$. Fix $y \in Y$ and set 
$k=\|y\|$. Assume that $y \notin \mathbb{Q}\left(B_{X} \cap K\right)$. Then, setting

$$
f(x)=\rho_{|\cdot|}(y, \mathbb{Q}(x))=\inf \{|y-v| \mid v \in \mathbb{Q}(x)\},
$$

we see that $f(x)>0$ for all $x \in B_{K}=B_{X} \cap K$.

Applying the variational principle of Ekeland [16], we can find $w \in B_{K}$ such that $\|w\|<1$ and

$$
f(x)+(k+\varepsilon)\|x-w\|>f(w), \quad \forall x \in B_{K} .
$$

Let, for $n=1,2, \ldots$,

$$
D_{n}=\left\{y^{*} \in Y^{*}|| y^{*} \mid \leqslant 1, \inf _{v \in \mathbb{Q}(w)}\left\langle y^{*}, y-v\right\rangle>f(w)-(1 / n)\right\} .
$$

Then $D_{n}$ is a convex and weak* closed set decreasing as $n$ increases and $\left|y^{*}\right|>1$ $-(1 / n)$ if $y^{*} \in D_{n}$.

Take arbitrarily an $h \in K$ and choose for any $t>0$ a $y_{t}^{*} \in Y^{*}$ with $\left|y_{t}^{*}\right|=1$ such that $\inf _{v \in \mathbb{Q}(w+t h)}\left\langle y_{t}^{*}, y-v\right\rangle=f(w+t h)$. Since $\mathbb{Q}(w+t h)$ Hausdorff converges to $\mathcal{Q}(w)$ as $t \rightarrow 0$ (according to 1.12$), y_{t}^{*} \in D_{n}$ if $t$ is sufficiently small.

We have for $h \in K$

$$
\begin{aligned}
f(w+t h)-f(w) & <\inf _{v \in \mathcal{Q}(w+t h)}\left\langle y_{t}^{*}, y-v\right\rangle-\inf _{v \in \mathscr{Q}(w)}\left\langle y_{t}^{*}, y-v\right\rangle \\
& =s\left(y_{t}^{*}, w\right)-s\left(y_{t}^{*}, w+t h\right)\left\langle t \cdot s\left(y_{t}^{*},-h\right)\right. \\
& \leqslant t \sup _{y^{*} \in D_{n}} s\left(y^{*},-h\right)
\end{aligned}
$$

which gives along with (1)

$$
\sup _{y^{*} \in D_{n}} s\left(y^{*},-h\right)+(k+\varepsilon)\|h\|>0, \quad \forall h \in K
$$

or $k+\varepsilon \geqslant \operatorname{sl}(\mathbb{Q},-K,|\cdot|)>\operatorname{Sl}(\mathbb{Q},-K)-\varepsilon$. Since $\varepsilon$ is arbitrary, this shows that $\|y\|=k \geqslant \operatorname{Sl}(\mathbb{Q},-K)$. In other words, if $\|y\|<\operatorname{Sl}(\mathbb{Q},-K)$, then $y \in \mathbb{Q}(x)$ for some $x \in B_{X} \cap K$.

6.8. Corollary. For a bounded fan $\mathbb{Q}$ from $X$ into $Y$ and a closed cone $K \subset X$, the inclusion $C(\mathbb{Q},-K) U_{Y} \subset \mathbb{Q}\left(B_{X} \cap K\right)$ is valid if either of the following three conditions is satisfied:

(a) there is an equivalent locally uniformly convex norm in $Y^{*}$;

(b) $\mathbb{Q}$ is norm-compact-valued and there is an equivalent strictly convex norm in $Y^{*}$;

(c) $K$ is convex and $\mathbb{Q}$ is single-valued.

Proof. Apply 5.19 and 5.15.

6.9. Corollary (BANACH OPEN MAPPING THEOREM). Let $A: X \rightarrow Y$ be a linear bounded operator and $K \subset X$ a closed convex cone such that $A(K)=Y$. Then the restriction of $A$ to $K$ is an open mapping.

Proof. We have $C(\mathscr{Q}, K)=C(\mathscr{Q},-K)>0$ (in view of 6.1). If now $x \in K$, $y=A x$ then for any $\varepsilon>0$,

$$
A\left(x+\varepsilon B_{K}\right)=A x+\varepsilon A\left(B_{K}\right) \supset A x+\varepsilon C(A, K) U_{Y}=C(A, K) U(x, \varepsilon) .
$$


6.10. Remark. It is easy to see that the proof in 6.7 implies a similar fact for fans: $\mathbb{Q}(x)+\operatorname{Sl}(\mathbb{Q},-K) U_{Y} \subset \mathbb{Q}\left(x+B_{K}\right)$.

6.11. Corollary (Robinson). Any closed convex process $\mathbb{Q}$ from $X$ into $Y$ with $\operatorname{dom} \mathcal{Q}=K$ and $\mathcal{Q}(K)=Y$ is open on $K$.

Proof. Apply 2.3 and 6.9.

6.12. REMARK. It is interesting to compare 6.2 and 6.9. First we observe that 6.2 is valid with $C(\mathbb{Q},-K)$ instead of $C(\mathbb{Q}, K)$ (it suffices to choose $f(x)$ in such a way that $|f(x)-f(-x)|<\varepsilon$ for all $x$, using the fact that $\mathbb{Q}(x) \cap \mathbb{Q}(-x) \neq \varnothing)$ so that there is no contradiction with 6.7. The converse replacement in 6.7 is questionable. Note further that $K$ need not be convex in 6.7, and 6.7 does not imply a corollary like 6.3.

7. Fans and linear operators. All linear operators in this section are continuous.

7.1. Definition. A linear operator $A$ is a linear selection of a fan $\mathbb{Q}$ if $A x \in \mathbb{Q}(x)$ for all $x$. The fan $\mathbb{Q}$ has the Hahn-Banach extension property (resp. the $k$-extension property) if any linear selection of the restriction of $\mathcal{Q}$ to a subspace of $X$ (resp. to a subspace of dimension $k$ ) can be extended to a selection of $Q$ defined on all of $X$.

The 0-extension property is simply the existence of a linear selection, and the 1 -extension property means that the fan is generated by a set of linear operators. Observe that a fan having the $k$-extension property $(k>1)$ is necessarily odd.

It is possible to describe fans having the Hahn-Banach extension property. Roughly speaking, they are fans whose values are bounded order intervals in certain conditionally complete ordered vector spaces: Concerning other extension properties, there are more questions than results. This section contains several sufficiently simple facts. It turns out in particular that even in the finite-dimensional spaces there are odd fans not generated by sets of linear operators.

7.2. Proposition. Let $L \subset X$ be a linear subspace of codimension one, let $e \in L$, and let $\mathcal{Q}$ be an odd fan from $X$ into $Y$. Let a linear operator $A: L \rightarrow Y$ be a linear selection of the restriction of $\mathbb{Q}$ on $L$. A necessary and sufficient condition for the existence of a linear selection of $\mathbb{Q}$ coinciding with $A$ on $L$ is that

$$
\bigcap_{x \in L}(\mathscr{Q}(x+e)-A x) \neq \varnothing \text {. }
$$

Proof. Assume that such a linear selection $B$ does exist. Then, for any $x \in L$, $\mathcal{Q}(x+e)-A x \ni B(x+e)-A x=B e$.

Conversely, let $y \in \cap_{x \in L}(\mathbb{Q}(x+e)-A x)$. Define $B$ by

$$
B(x+t e)=A x+t y \quad \text { for all } x \in L, t \in R .
$$

Then $B$ is a linear operator from $X$ into $Y$ which coincides with $A$ on $L$. We also have, for $x \in L$ and $t \neq 0$,

$$
B(x+t e)=t(A(x / t)+y) \subset t(A(x / t)+\mathbb{Q}((x / t)+e)-A(x / t))=\mathbb{Q}(x+t e) .
$$


7.3. Corollary. Let $\mathbb{Q}$ be an odd bounded fan from $X$ into $R^{k}$. Then the following three statements are equivalent:

(a) $\mathbb{Q}$ has the Hahn-Banach extension property;

(b) $Q$ has the k-extension property;

(c) whenever $A$ is a linear selection of the restriction of $\mathcal{Q}$ on an $r$-dimensional subspace $L$ of $X(r \geqslant k)$,

$$
\bigcap_{i=0}^{k}\left(\mathscr{Q}\left(x_{i}+e\right)-A x_{i}\right) \neq \varnothing, \quad \forall x_{0}, \ldots, x_{k} \in L, \forall e \notin L .
$$

Proof. Implications (a) $\Rightarrow$ (b) $\Rightarrow$ (c) are obvious. Let $L \subset X$ be a subspace, and let $B: L \rightarrow R^{k}$ be a linear selection of the restriction of $\mathcal{Q}$ on $L$. If $e \notin L$, then combining (c), the theorem of Helley and Proposition 7.2, we conclude that there is an extension of $B$ to the space generated by $L \cup\{e\}$ which is a linear selection of Q. Applying the standard transfinite induction arguments, we arrive at (a).

We proceed now with constructing the example of a finite-dimensional odd fan without the 1-extension property.

7.4. Proposition. Let $L$ and $e$ be the same as in 7.2. Assume that we are given two set-valued mappings from $L$ into $Y$, an odd fan $\mathscr{B}$ and another mapping $\mathcal{C}$ with convex, closed and bounded values. A necessary and sufficient condition for the existence of an odd fan $\mathbb{Q}$ from $X$ into $Y$ such that $\mathbb{Q}(x)=\mathscr{B}(x)$ and $\mathbb{Q}(x+e)=$ $\mathcal{C}(x)$ for $x \in L$ is that

$$
\begin{gathered}
\mathscr{B}(h) \subset \overline{\mathcal{C}(x+h)-\mathcal{C}(x)}, \\
\mathcal{C}(x+h) \subset \overline{\mathcal{C}(x)+\mathscr{B}(h)}, \\
\mathcal{C}(\alpha u+(1-\alpha) x) \subset \overline{\alpha \mathcal{C}(u)+(1-\alpha) \mathcal{C}(x)}
\end{gathered}
$$

for all $x, u, h \in L, 0 \leqslant \alpha \leqslant 1$.

Proof. If such a fan exists, then

$$
\begin{aligned}
\mathscr{B}(h) & =\mathbb{Q}(h) \subset \overline{\mathbb{Q}(e+x+h)+\mathbb{Q}(-e-x)} \\
& =\overline{\mathbb{Q}(e+x+h)-\mathbb{Q}(e+x)}=\overline{\mathcal{C}(x+h)-\mathcal{C}(x)}, \\
\mathcal{C}(x+h) & =\mathbb{Q}(e+x+h) \subset \overline{\mathbb{Q}(e+x)+\mathbb{Q}(h)}=\overline{\mathcal{C}(x)+\mathscr{B}(h)}, \\
\mathcal{C}(\alpha u+(1-\alpha) x) & =\mathbb{Q}(\alpha(e+u)+(1-\alpha)(e+x)) \\
& \subset \overline{\alpha \mathscr{Q}(e+u)+(1-\alpha) \mathscr{Q}(e+x)} \\
& =\overline{\alpha \mathcal{C}(u)+(1-\alpha) \mathcal{C}(x)} .
\end{aligned}
$$

Assume now that (1)-(3) hold. Then we set, for $u=u(t)=x+t e, x \in L$, $t \in R$,

$$
\mathscr{Q}(u)=\left\{\begin{array}{l}
\mathscr{B}(x), \quad \text { if } t=0 \\
t \mathcal{C}(x / t), \quad \text { if } t \neq 0
\end{array}\right.
$$

We must show that

$$
\mathscr{Q}(\lambda u)=\lambda \mathscr{Q}(u), \quad \mathbb{Q}\left(u_{1}+u_{2}\right) \subset \overline{\mathbb{Q}\left(u_{1}\right)+\mathscr{Q}\left(u_{2}\right)} .
$$


The first of these relations is obvious. To prove the second, we shall consider four possible situations:

(a) if $t_{1}=t_{2}=0$, then it follows from the fact that $\mathscr{B}$ is a fan;

(b) if $t_{1}+t_{2}=0$, i.e. if $t_{1}=-t_{2}=t$, then taking $x=-x_{2} / t$ and $h=$ $\left(x_{1}+x_{2}\right) / t$, we obtain from (1)

$$
\begin{aligned}
\mathscr{Q}\left(u_{1}+u_{2}\right) & =\mathscr{B}\left(x_{1}+x_{2}\right)=t \mathscr{B}(h) \subset \overline{t(\mathcal{C}(x+h)-\mathcal{C}(x))} \\
& =\overline{t \mathcal{C}\left(x_{1} / t\right)-t \mathcal{C}\left(-x_{2} / t\right)}=\overline{\mathscr{Q}\left(u_{1}\right)+\mathscr{Q}\left(u_{2}\right)} ;
\end{aligned}
$$

(c) if $t_{1}+t_{2} \neq 0$ and $t_{1} t_{2} \geqslant 0$, say, both are nonnegative, then taking $\alpha=$ $t_{1} /\left(t_{1}+t_{2}\right)$, we have from (3) (or from (2) if one of $t_{i}$ is zero)

$$
\begin{aligned}
& \mathcal{Q}\left(u_{1}+u_{2}\right)=\left(t_{1}+t_{2}\right) \mathcal{C}\left(\left(x_{1}+x_{2}\right) /\left(t_{1}+t_{2}\right)\right) \\
& =\left\{\begin{array}{l}
\left(t_{1}+t_{2}\right) \mathcal{C}\left(\alpha\left(x_{1} / t_{1}\right)+(1-\alpha)\left(x_{2} / t_{2}\right)\right), \quad \text { if } t_{2} \neq 0, \\
t_{1} \mathcal{C}\left(\left(x_{1}+x_{2}\right) / t_{1}\right), \quad \text { if } t_{2}=0,
\end{array}\right.
\end{aligned}
$$

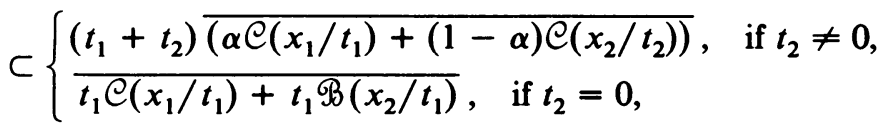

$$
\begin{aligned}
& = \begin{cases}\overline{t_{1} \mathcal{C}\left(x_{1} / t_{1}\right)+t_{2} \mathcal{C}\left(x_{2} / t_{2}\right)}, & \text { if } t_{2} \neq 0, \\
t_{1} \mathcal{C}\left(x_{1} / t_{1}\right)+\mathscr{B}\left(x_{2}\right) & \text { if } t_{2}=0,\end{cases} \\
& =\overline{\mathscr{Q}\left(u_{1}\right)+\mathscr{Q}\left(u_{2}\right)} \text {; }
\end{aligned}
$$

(d) if $t_{1} t_{2}<0$, say $t_{1}>0>t_{2}, t_{1} \geqslant\left|t_{2}\right|$, then taking $t=\left|t_{2}\right|, s=t_{1}-t, k=t_{1} / t$ $=1+s / t$, we have $u_{1}=k u^{\prime}$, where $u^{\prime}=\left(x_{1} / k\right)+t e$ and $u_{2}=x_{2}-t e$. Using subsequently (c), (b) and the homogeneity of $\mathbb{Q}$, we have

$$
\begin{aligned}
\mathbb{Q}\left(u_{1}+u_{2}\right) & =\mathbb{Q}\left((k-1) u^{\prime}+u^{\prime}+u_{2}\right) \\
& \subset \bar{Q}\left((k-1) u^{\prime}\right)+\mathbb{Q}\left(u^{\prime}+u_{2}\right) \\
& \subset \overline{(k-1) \mathbb{Q}\left(u^{\prime}\right)+\mathbb{Q}\left(u^{\prime}\right)+\mathbb{Q}\left(u_{2}\right)} \\
& =\overline{\mathbb{Q}\left(u_{1}\right)+\mathbb{Q}\left(u_{2}\right)} .
\end{aligned}
$$

7.5. Proposition. Let $Q, P_{-1}, P_{0}, P_{1}$ be nonempty bounded closed convex subsets in $R^{n}$ such that $Q \subset P_{1}-P_{0}, Q \subset P_{0}-P_{-1}, 2 Q \subset P_{1}-P_{-1}, P_{1} \subset P_{0}+Q$, $P_{0} \subset P_{-1}+Q, P_{0} \subset P_{1}-Q, P_{-1} \subset P_{0}-Q, 2 P_{0} \subset P_{1}+P_{-1}$. Then the set-valued mappings

$$
\begin{aligned}
& \mathscr{B}(t)=t Q, \\
& \Theta(t)=\left\{\begin{array}{l}
P_{\text {sign } t}+(\operatorname{sign} t)(|t|-1) Q, \quad \text { if }|t|>1, \\
|t| P_{\text {sign } t}+(1-|t|) P_{0}, \text { if }|t|<1
\end{array}\right.
\end{aligned}
$$

satisfy conditions (1)-(3).

Proof. We must show that for any $t, s \in R, \alpha \in[0,1]$,

$$
\begin{gathered}
(t-s) Q \subset \mathcal{C}(t)-\mathcal{C}(s), \\
\mathcal{C}(t) \subset \mathcal{C}(s)+(t-s) Q, \\
\mathcal{C}(\alpha t+(1-\alpha) s) \subset \alpha \mathcal{C}(t)+(1-\alpha) \mathcal{C}(s) .
\end{gathered}
$$


In view of the symmetry, this can be done only for $t>0, t>|s|$.

If $|s| \geqslant 1$, then

$$
\begin{aligned}
e(t)-e(s) & =P_{1}+(t-1) Q-P_{\text {sign } s}-(\operatorname{sign} s)(|s|-1) Q \\
& \supset(1-\operatorname{sign} s) Q+(t-1) Q-(\operatorname{sign} s)(|s|-1) Q \\
& \supset(1-\operatorname{sign} s+t-1-s+\operatorname{sign} s) Q=(t-s) Q, \\
e(s)+(t-s) Q & =P_{\text {sign } s}+(\operatorname{sign} s)(|s|-1) Q+(t-s) Q \\
& \supset P_{\operatorname{sign} s}+(s-\operatorname{sign} s+t-s) Q \\
& =P_{\text {sign } s}+(t-\operatorname{sign} s) Q \\
& =P_{\text {sign } s}+(1-\operatorname{sign} s) Q+(t-1) Q \\
& \supset P_{1}+(t-1) Q=C(t) .
\end{aligned}
$$

If $t>1,|s| \leqslant 1$, then

$$
\begin{aligned}
\mathcal{C}(t)-\mathcal{C}(s) & =P_{1}+(t-1) Q-|s| P_{\text {sign } s}+(|s|-1) P_{0} \\
& =|s|\left(P_{1}-P_{\text {sign } s}\right)+(1-|s|)\left(P_{1}-P_{0}\right)+(t-1) Q \\
& \supset|s|(1-\operatorname{sign} s) Q+(1-|s|) Q+(t-1) Q \\
& \supset(|s|-s+1-|s|+t-1) Q=(t-s) Q, \\
C(s)+(t-s) Q & =|s| P_{\text {sign } s}+(1-|s|) P_{0}+(t-s) Q \\
& =|s| P_{\text {sign } s}+|s|(1-\operatorname{sign} s) Q+(1-|s|)\left(P_{0}+Q\right)+(t-1) Q \\
& \supset|s| P_{1}+(1-|s|) P_{1}+(t-1) Q \\
& =P_{1}+(t-1) Q=C(t) .
\end{aligned}
$$

If $|t|<1$, then

$$
\begin{aligned}
\mathcal{C}(t)-\mathcal{C}(s) & =t P_{1}+(1-t) P_{0}-|s| P_{\text {sign } s}-(1-|s|) P_{0} \\
& \supset(t-|s|) P_{1}+|s|\left(P_{1}-P_{\text {sign } s}\right)-(t-|s|) P_{0} \\
& =(t-|s|)\left(P_{1}-P_{0}\right)+|s|\left(P_{1}-P_{\text {sign } s}\right) \\
& \supset(t-|s|) Q+|s|(1-\operatorname{sign} s) Q \supset(t-s) Q, \\
C(s)+(t-s) Q & =|s| P_{\text {sign } s}+(1-|s|) P_{0}+(t-s) Q \\
& =|s| P_{\text {sign } s}+(1-t) P_{0}+(t-|s|) P_{0}+(t-|s|) Q+(|s|-s) Q \\
& \supset|s| P_{1}+(t-|s|) P_{1}+(1-t) P_{0} \\
& =t P_{1}+(1-t) P_{0}=C(t) .
\end{aligned}
$$

This proves the first two inclusions. To prove the third one it suffices to show that, for any $y^{*} \in Y^{*}$, the support function $\delta^{*}\left(y^{*}, \mathcal{C}(t)\right)$ is convex in $t$. We have

$$
\delta^{*}\left(y^{*}, \mathcal{C}(t)\right)=\left\{\begin{array}{l}
(t-1) \delta^{*}\left(y^{*}, Q\right)+\delta^{*}\left(y^{*}, P_{1}\right), \quad t>1, \\
t\left(\delta^{*}\left(y^{*}, P_{1}\right)-\delta^{*}\left(y^{*}, P_{0}\right)+\delta^{*}\left(y^{*}, P_{0}\right), \quad 0<t<1,\right. \\
t\left(\delta^{*}\left(y^{*}, P_{0}\right)-\delta^{*}\left(y^{*}, P_{-1}\right)+\delta^{*}\left(y^{*}, P_{0}\right), \quad-1<t<0,\right. \\
-(t-1) \delta^{*}\left(y^{*},-Q\right)+\delta^{*}\left(y^{*}, P_{-1}\right), \quad t<-1 .
\end{array}\right.
$$


This function is continuous and piecewise linear in $t$ and the slopes satisfy

$$
\begin{aligned}
-\delta^{*}\left(y^{*},-Q\right) & <\delta^{*}\left(y^{*}, P_{0}\right)-\delta^{*}\left(y^{*}, P_{-1}\right) \\
& <\delta^{*}\left(y^{*}, P_{1}\right)-\delta^{*}\left(y^{*}, P_{0}\right)<\delta^{*}\left(y^{*}, Q\right)
\end{aligned}
$$

which is immediate from the inclusions assumed.
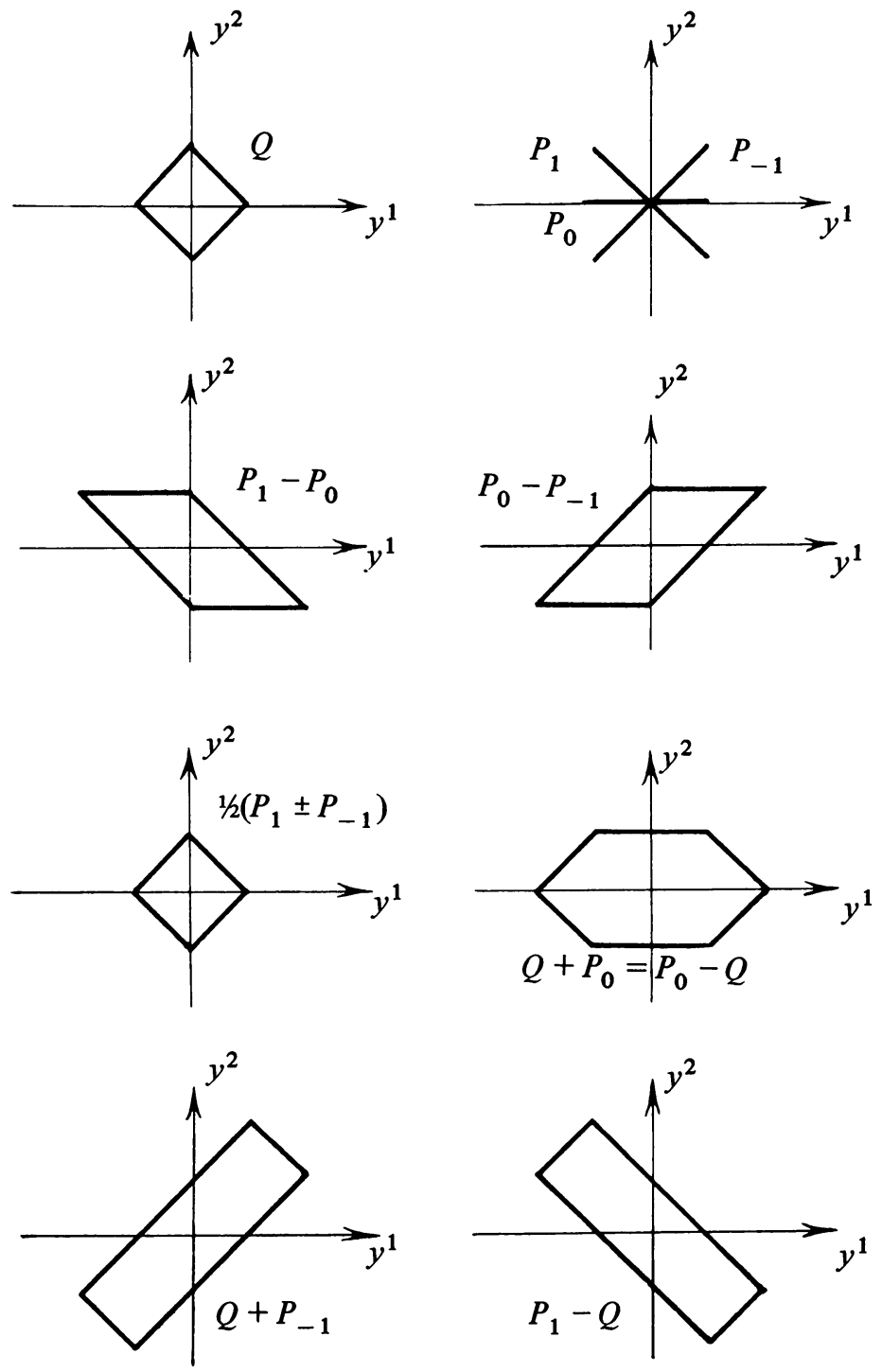

FIgURE 1

7.6. Example. Consider four plane sets $Q, P_{-1}, P_{0}, P_{1}$ as in Figure 1 . It is easily seen from the figure that all of the inclusions of 7.5 are satisfied in this case. The sets $P_{i}, i=-1,0,1$, contain the origin. Therefore by 7.4 there is an odd (and obviously bounded) fan $\mathbb{Q}(x)$ from $R^{2}$ into $R^{2}$ such that $\mathbb{Q}\left(e_{2}\right)=Q, \mathbb{Q}\left(i e_{2}+e_{1}\right)=$ $P_{i}(i=-1,0,1)$, where $e_{1}, e_{2}$ is a basis in $R^{2}$. It is easily seen from the picture that 
if $y \in Q$ and the first component of $y$ differs from zero, then $\left(P_{-1}+y\right) \cap P_{0} \cap$ $\left(P_{1}-y\right)=\varnothing$ and Corollary 7.3 shows that there is no linear selection $A$ of $\mathscr{Q}$ such that $A e_{2}=y$. Thus the fan is not generated by linear operators.

Observe that the fan does have the 0-extension property because the zero operator is a linear selection of $\mathcal{Q}$. It is an interesting question, does there exist an odd bounded fan in a finite-dimensional space which has no linear selections?

The final result of this section concerns the $k$-extension property for arbitrary $k$.

7.7. TheOREM. Let $X$ and $Y$ be locally convex linear Hausdorff topological spaces and $\mathcal{Q}$ an odd bounded fan from $X$ into $Y$ such that all sets $\mathcal{Q}(x)$ are compact and the restriction of $\mathbb{Q}$ to every finite-dimensional subspace of $X$ has the $k$-extension property. Then $\mathbb{Q}$ has the $k$-extension property.

Proof. Let $L_{0}$ be a subspace of $X$ with $\operatorname{dim} L_{0} \leqslant k$, and let $A_{0}: L_{0} \rightarrow Y$ be a linear selection of the restriction of $\mathscr{Q}$ on $L_{0}$. Let $\left\{L_{\xi}\right\}_{\xi \in \Xi}$ be the collection of all finite-dimensional subspaces of $X$ containing $L_{0}$ which is completely ordered in a certain way (so that $\Xi$ is just the collection of all ordinals whose cardinality does not exceed card $X$ ). To prove the theorem, it is sufficient to find a family $\left\{A_{\xi}\right\}$ of linear selections of the restrictions of $\mathcal{Q}$ on $L_{\xi}$ having the following properties:

(a) the restriction of every $A_{\xi}$ on $L_{0}$ coincides with $A_{0}$;

(b) for any finite collection $\left\{\xi_{1}, \ldots, \xi_{k}\right\}$ there is a linear operator $A: \sum L_{\xi_{k}} \rightarrow Y$ which is a linear selection of the restriction of $\mathscr{Q}$ to the sum and satisfies $A x=A_{\xi_{i}} x$ whenever $x \in L_{\xi_{i}}$.

Indeed, in this case, the relation

$$
A x=A_{\xi} x, \text { if } x \in L_{\xi},
$$

uniquely defines an operator from $X$ into $Y$ such that $A x \in \mathbb{Q}(x)$ for all $x$ and $A x=A_{0} x$ for $x \in L_{0}$.

Assume that, for a given ordinal $\eta$, we have already defined operators $A_{\xi}$ : $L_{\xi} \rightarrow Y$ for $\xi<\eta$ which satisfy (a) and the following weak form of (b):

$\left(b_{\eta}^{\prime}\right)$ for any finite collection $\left\{\xi_{1}, \ldots, \xi_{k}\right\}$ there is a linear operator $A: \Sigma L_{\xi_{1}} \rightarrow Y$ which is a linear selection of the restriction of $\mathcal{Q}$ to the sum and satisfies $A x=A_{\xi_{i}} x$ whenever $\xi_{i}<\eta, x \in L_{\xi_{i}}$.

Denote by $Q\left(\xi_{1}, \ldots, \xi_{k}\right)$ the collection of such operators, and let $P\left(\xi_{1}, \ldots, \xi_{k}\right)$ be the collection of restrictions of elements of $Q\left(\eta, \xi_{1}, \ldots, \xi_{k}\right)$ on $L_{\eta}$.

Then every $Q\left(\xi_{1}, \ldots, \xi_{k}\right)$ is compact in the topology of compact convergence. Indeed, inasmuch as $\mathscr{Q}$ is a bounded fan, every $Q\left(\xi_{1}, \ldots, \xi_{k}\right)$ is equicontinuous as a collection of linear selections of $\mathcal{Q}$, and the assumption that the values of $\mathscr{Q}$ are compact implies compactness of the set which is obviously closed in the topology of pointwise convergence.

It follows that every $P\left(\xi_{1}, \ldots, \xi_{k}\right)$ is also compact in the topology of pointwise convergence. On the other hand,

$$
P\left(\xi_{1}, \ldots, \xi_{k}, \eta_{1}, \ldots, \eta_{s}\right) \subset P\left(\xi_{1}, \ldots, \xi_{k}\right) \cap P\left(\eta_{1}, \ldots, \eta_{s}\right)
$$

for any $\xi_{1}, \ldots, \xi_{k}, \eta_{1}, \ldots, \eta_{s}$, which is obvious. 
Thus there is a linear operator $A_{\eta}: L_{\eta} \rightarrow Y$ which belongs to all $P\left(\xi_{1}, \ldots, \xi_{k}\right)$. This means that $A_{\eta}$ is a linear selection of the restriction of $\mathcal{Q}$ on $L_{\eta}$ and for any $\xi_{1}, \ldots, \xi_{k}$ there is $A \in Q\left(\eta, \xi_{1}, \ldots, \xi_{k}\right)$ such that the restriction of $A$ to $L_{\eta}$ coincides with $A_{\eta}$. We have proved therefore that if $\left(\mathrm{b}_{\xi}^{\prime}\right)$ holds for all $\xi<\eta$, then $\left(b_{\eta}^{\prime}\right)$ also holds. Observe, finally, that $\left(b_{0}^{\prime}\right)$ is valid according to the assumptions of the theorem. This completes the induction, thereby proving the theorem.

\section{Differential calculus}

In this chapter all spaces are Banach.

8. Directional derivatives, tangent and normal cones. We begin this chapter by surveying some definitions and results (which are partly new) connected with concepts of directional derivative and tangent and normal cone.

8.1. Definition. Let $f(x)$ be a real-valued continuous function defined in a neighbourhood of $z \in X$. We set for any $\varepsilon>0$ :

$$
\begin{aligned}
& d_{e}{ }^{-} f(z ; h)=\inf \left\{t^{-1}(f(z+t h)-f(z)) \mid 0<t\|h\|<\varepsilon, t>0\right\}, \\
& d_{\varepsilon}{ }^{+} f(z ; h)=\sup \left\{t^{-1}(f(z+t h)-f(z)) \mid 0<t\|h\|<\varepsilon, t>0\right\}, \\
& d_{e}^{0} f(z ; h)=\sup \left\{t^{-1}(f(x+t h)-f(x)) \mid\|x-z\|<\varepsilon, t>0,\|x+t h-z\|<\varepsilon\right\} .
\end{aligned}
$$

Clearly, all three functions, as functions of $h$, are positively homogeneous of degree one (i.e. satisfy $g(t h)=\operatorname{tg}(h)$ ), $d_{\varepsilon}^{-}$is u.s.c. in $h$ and the other two are 1.s.c. in $h$. It is also obvious that $d_{\varepsilon}{ }^{-} f(z ; h)<d_{\varepsilon}{ }^{+} f(z ; h)<d_{\varepsilon}^{0}(z ; h)$ and that dependence on $\varepsilon$ in all three cases is monotone (nondecreasing in the first and nonincreasing in the other two). We set further

$$
\begin{aligned}
& d^{-} f(z ; h)=\liminf _{\substack{u \rightarrow h \\
e \downarrow 0}} d_{e}^{-} f(z ; u), \\
& d^{+} f(z ; h)=\lim _{\substack{u \rightarrow h \\
e \downarrow 0}} d_{e}^{+} f(z ; u), \\
& d^{0} f(z ; h)=\lim _{e \downarrow 0} d_{e}^{0} f(z ; h) .
\end{aligned}
$$

We refer to [3], [45] for more details concerning the first two directional derivatives and to [8]-[12] for everything concerning the last one.

Observe that if $f$ is Lipschitz, then all functions above are Lipschitz in $h$ (with the same constant as $f$ ) and $d^{0}$, in addition, is u.s.c. in both variables (it is always u.s.c. in $z$ ). In this case the definitions of $d^{-}$and $d^{+}$can be simplified as follows:

$$
d^{-} f(z ; h)=\lim _{\varepsilon \downarrow 0} d_{\varepsilon}^{-} f(z ; h), \quad d^{+} f(z ; h)=\lim _{\varepsilon \downarrow 0} d_{\varepsilon}^{+} f(z ; h) .
$$

8.2. Proposition. The function $h \rightarrow d_{e}^{0} f(z ; h)$ is convex (hence so is $d^{0} f(z ; h)$ ).

Proof. Note that $d_{\varepsilon}^{0} f(z ; h)>-\infty$ for all $h$. We consider, for simplicity, only the case when this function is finite for all $h$. The extension to the general case is not difficult.

Since $d_{e}^{0} f(z ; \cdot)$ is homogeneous, it suffices to prove that

$$
d_{e}^{0} f(z ; e+h) \leqslant d_{e}^{0} f(z ; e)+d_{e}^{0} f(z ; h)
$$


for all $e, h$. We have by definition $d_{\varepsilon-\delta}^{0} f(z ; h)<d_{e}^{0} f(z ; h), \forall h \in X, \forall \delta>0$. Let $x$, $h$ and $t>0$ be such that $\|x-z\|<\varepsilon,\|x+t h-z\|<\varepsilon$ and

$$
d_{e}^{0} f(z ; h) \leqslant t^{-1}(f(x+t h)-f(x))+\sigma .
$$

Then there is $\delta>0$ such that $\|x-z\|<\varepsilon-\delta,\|x+t h-z\|<\varepsilon-\delta$ and hence $d_{e}^{0} f(z ; h) \leqslant d_{\varepsilon-\delta}^{0} f(z ; h)+\sigma$ and to prove (1), it suffices to show that, for any $\delta>0$, $\sigma>0$,

$$
d_{\varepsilon-\delta}^{0} f(z ; e+h) \leqslant d_{\varepsilon}^{0} f(z ; e)+d_{e}^{0} f(z ; h)+\sigma, \quad \forall e, h .
$$

So let us fix some $e$ and $h$, and let $x$ and $t>0$ be such that $\|x-z\|<\varepsilon-\delta$, $\|x+t(e+h)-z\|<\varepsilon-\delta$ and

$$
d_{\varepsilon-\delta}^{0} f(z ; e+h)<t^{-1}(f(x+t(e+h))-f(x))+\sigma / 2 .
$$

Since $f$ is continuous, for any $\tau>0, \tau<t$, there are $0<t^{\prime}<\tau$ and $x^{\prime}$ belonging to the line segment $[x, x+t(e+h)]$ such that $x^{\prime}+t^{\prime}(e+h)$ also belongs to this segment and $t^{\prime-1}\left(f\left(x^{\prime}+t^{\prime}(e+h)\right)-f\left(x^{\prime}\right)\right)>t^{-1}(f(x+t(e+h))-f(x))-\sigma / 2$.

Choose $\tau$ so small that $\left\|x^{\prime}+t^{\prime} h-z\right\|<\varepsilon$. Then

$$
\begin{aligned}
d_{e-\delta}^{0} f(z ; e+h)-\sigma \leqslant & t^{\prime-1}\left(f\left(x^{\prime}+t^{\prime}(e+h)\right)-f\left(x^{\prime}\right)\right) \\
= & t^{\prime-1}\left(f\left(x^{\prime}+t^{\prime}(e+h)\right)-f\left(x^{\prime}+t^{\prime} h\right)\right) \\
& +t^{\prime-1}\left(f\left(x^{\prime}+t^{\prime} h\right)-f\left(x^{\prime}\right)\right) \\
\leqslant & d_{e}^{0} f(z ; e)+d_{\varepsilon}^{0} f(z ; h) .
\end{aligned}
$$

8.3. Definition. Let $z \in S \subset X$. Consider the following cones:

$$
\begin{aligned}
K_{\varepsilon}(S, z) & =\left\{h \mid d_{\varepsilon}^{-} \rho(z, S ; h)=0\right\}, \\
T_{\varepsilon}(S, z) & =\left\{h \mid d_{\varepsilon}^{-} \rho(z, S ; h)<\varepsilon\|h\|\right\}, \\
\tilde{T}_{\varepsilon}(S, z) & =\bigcap_{\delta>0} \bigcup_{\|x-z\|<\delta} T_{\varepsilon}(S, x), \\
T_{1}(S, z) & =\bigcap_{\varepsilon>0} \tilde{T}_{\varepsilon}(S, z), \\
T(S, z) & =\left\{h \mid d^{-} \rho(z, S ; h)=0\right\}, \\
T_{c}(S, z) & =\left\{h \mid d^{0} \rho(z, S ; h)=0\right\} .
\end{aligned}
$$

(Since all these cones are defined with the help of the distance function, there is no loss of generality in assuming that $S$ is closed which we shall always do.)

The cone $T(S, z)$ is the tangent cone to $S$ at $z$, as has been known for a long time (see [3], [35], [45] and references there); $T_{c}(S, z)$ is the Clarke tangent cone ([8]-[12], [22], [49]). As is well known, $h \in T(S, z)$ if and only if there are sequences $t_{n} \downarrow 0$ and $h_{n} \rightarrow h$ such that $z+t_{n} h_{n} \in S$ for all $n$ (which is obvious) and $h \in T_{c}(S, z)$ if and only if, for any sequences $t_{n} \downarrow 0$ and $x_{n} \rightarrow z$, there is a sequence $h_{n} \rightarrow h$ such that $z+t_{n} h_{n} \in S$ for all $n$ [22].

Obviously, $T_{c}(S, z) \subset T(S, z) \subset T_{e}(S, z), \quad T_{1}(S, z) \subset \tilde{T}_{e}(S, z) \subset T_{e}(S, z)$, $T(S, z) \subset K_{e}(S, z)$ and

$$
K_{\varepsilon}(S, z)=\overline{\bigcup_{t>0} t^{-1}[(S-z) \cap B(0, \varepsilon)]}
$$

is the cone generated by $(S-z) \cap B(0, \varepsilon)$. 
Observe in this connection that, since $\rho(x, S)$ is a Lipschitz function, all cones introduced above are closed and the Clarke tangent cone is convex.

8.4. Proposition. $T_{c}(S, z) \subset T_{1}(S, z)$.

Proof. Consider the function $g(h)=\lim _{\delta \rightarrow 0} \sup _{\|x-z\|<\delta} d^{-} \rho(x, S ; h)$. Then

$$
\begin{aligned}
& \tilde{T}_{e}(S, z)=\{h \mid g(h) \leqslant \varepsilon\|h\|\}, \\
& T_{1}(S, z)=\{h \mid g(h)=0\} .
\end{aligned}
$$

On the other hand, $g(h) \leqslant d^{0} \rho(z, S ; h)$ and the inclusion follows.

8.5. Proposition. $T(S, z)=\cap_{e>0} K_{e}(S, z)$. If, in addition, $\operatorname{dim} X<\infty$, then

$$
\lim _{\varepsilon \downarrow 0} H\left(K_{e}(S, z) \cap B_{X}, T(S, z) \cap B_{X}\right)=0 .
$$

Proof. If $h \in \cap K_{e}(S, z), h \neq 0$, then for any $n=1,2, \ldots$, there are $t_{n} \downarrow 0$ and $h_{n} \in t_{n}^{-1}[(S-z) \cap B(0,1 / n)]$ such that $\left\|h-h_{n}\right\|<1 / n$ so that $z+t_{n} h_{n} \in S$ and $t_{n} h_{n} \in B(0,1 / n)$; hence $t_{n} \leqslant\left\|h_{n}\right\|^{-1} / n \rightarrow 0$; hence $h \in T(S, z)$. Together with the last inclusion in 8.3 , this gives the required equality.

The second part of the proposition follows from the fact that a decreasing sequence of compact sets in a metric space Hausdorff converges to the intersection.

8.6. REMARK. It is reasonable to ask why it is necessary to have so many concepts of tangent cones. The answer is that tangent cones usually enter conclusions of various theorems in such a way that a certain property is claimed to be valid for all elements of the cone. Thus the larger the cone involved, the stronger is the result. This prompts us to seek, in each particular case, the largest possible cone for which the desired result can be proved or which can be calculated in a given situation.

If $S$ is sufficiently smooth about $z$, then all infinitesimal tangent cones (those without the subscript $\varepsilon$ ) coincide but, generally, they are different. Proposition 8.5 shows that, in a finite-dimensional space, the tangent cone $T(S, z)$ gives the best possible first order approximation for $S$ at $z$. Things change if $\operatorname{dim} X=\infty$ and one can easily find simple examples when all the cones except $K_{e}(S, z)$ (which always differs from $\{0\}$ if $z$ is not an isolated point) are trivial, that is to say, contain only the zero element. (For instance, let $S$ be the graph of the mapping $f:[0,1] \rightarrow$ Hilbert space which is linear on every segment $[1 /(n+1), 1 / n], n=1,2, \ldots$, equals zero at 0 and $e_{n} / n$ at $1 / n$, where $\left\{e_{k} \mid k=1,2, \ldots\right\}$ is an orthonormal basis. The mapping is obviously Lipschitz but no nontrivial tangent cone to its graph exists at $z=(0,0)$.)

8.7. Definition. Let $f$ be a real-valued Lipschitz function defined in a neighbourhood of $z$. We set

$$
\partial_{c} f(z)=\left\{x^{*} \in X^{*} \mid\left\langle x^{*}, h\right\rangle\left\langle d^{0} f(z ; h), \forall h \in X\right\} .\right.
$$

This is the generalized gradient of Clarke. It is always a nonempty convex weak* compact set.

Given a closed set $S \subset X$ containing $z$, the cone

$$
N_{c}(S, z)=\bigcup_{\lambda>0} \lambda \partial_{c} \rho(S, z)
$$


is the $C$-normal cone to $S$ at $z$. It is also convex and the polar of $N_{c}(S, z)$ coincides with $T_{c}(S, z)$.

We refer to [8]-[12] for more details.

8.8. Definition. Let $f$ be a continuous function defined in a neighbourhood of $z$. We set

$$
\partial_{\varepsilon}^{-} f(z)=\left\{x^{*} \in X^{*} \mid\left\langle x^{*}, h\right\rangle\left\langle d^{-} f(z, h)+\varepsilon\|h\|, \forall h \in X\right\}\right.
$$

and

$$
\partial_{m} f(z)=\bigcap_{\delta>0} \bigcap_{\varepsilon>0} \overline{\bigcup_{\|x-z\|<\delta} \partial_{\varepsilon}^{-} f(x)} .
$$

The latter will be called the $M$-subdifferential of $f$ at $z$.

If $S \subset X$ is closed and $z \in S$, then the cone $N_{m}(S, z)=\cup_{\lambda>0} \lambda \partial_{m} \rho(z, S)$ will be called the $M$-normal cone to $S$ at $z$.

This cone is closely related to (and is perhaps even smaller than) the normal cone introduced by Mordukhovich [42], [43], [33].

8.9. Proposition. If $f$ is a Lipschitz function, then $\partial_{m} f(z) \subset \partial_{c} f(z)$ and therefore

$$
N_{m}(S, z) \subset N_{c}(S, z) \text {. }
$$

If $\operatorname{dim} X<\infty$, then conv $\partial_{m} f(z)=\partial_{c} f(z)$ and therefore

$$
\operatorname{conv} N_{m}(S, z)=N_{c}(S, z) \text {. }
$$

Proof. The first part of the proposition follows from the fact that $d^{-} f(x ; h)<$ $d^{0} f(x ; h)$ for all $x, h$ and the second function is u.s.c. in $x$.

Now let $\operatorname{dim} X<\infty$. Then $f$ is everywhere differentiable up to a set of Lebesgue measure zero. Let $\delta f(z)$ denote the collection of all limits of sequences $f^{\prime}\left(x_{n}\right)$ such that $x_{n} \rightarrow z$ and $f$ is differentiable at every $x_{n}$. As was shown by Clarke [8], the convex hull of $\delta f(z)$ coincides with $\partial_{c} f(z)$. On the other hand, every element of $\delta f(z)$ belongs to $\partial_{m} f(z)$ (because $d^{-} f(x ; h)=\left\langle f^{\prime}(x), h\right\rangle$ if $f$ is differentiable at $x$ ).

8.10. REMARK. Normal cones enter theorems usually in a dual way: as a rule it is claimed that there is a point in a normal cone having a certain property. Thus the smaller the normal cone, the stronger is the result.

From this point of view, $M$-normal cones seem to be the best possible in the finite-dimensional case though they may be less convenient to work with than, say, $C$-normal cones which are convex. But in an infinite-dimensional case we can have $N_{m}(S, z)=X^{*}$ even for a "well-connected" set.

We conclude this section with a rather surprising result concerning $M$-subdifferentials and $M$-normal cones (if one takes into account that both may be nonconvex). Basically, this result should be attributed to Kruger and Mordukhovich [33] who established the same result for the cones introduced by Mordukhovich and the corresponding subdifferentials.

8.11. Theorem. Assume that the norm in $X$ is Gâteaux differentiable. Let $f$ be a real-valued Lipschitz function defined in a neighbourhood of $z$. If $f$ attains a local minimum on $S$ at $z$, then

$$
0 \in \partial_{m} f(z)+N_{m}(S, z)
$$


Proof. Let $c>0$ be such that $B(z, c)$ belongs to the domain of $f$ and $f(x)>f(z)$ whenever $x \in S,\|x-z\| \leqslant c$. Let $L$ be a Lipschitz constant of $f$.

Consider the function $g_{r}(x, u)=f(x)+(r / 2)\|x-u\|^{2}$. It is easy to calculate that

$$
f(z) \leqslant \inf \left\{g_{r}(x, u) \mid x \in B(z, c), u \in S\right\}+k_{r},
$$

where $k_{r}=L / 2 r$, and $\lim \sup _{n \rightarrow \infty} \rho\left(x_{n}, S\right)=\lim \sup _{n \rightarrow \infty}\left\|x_{n}-u_{n}\right\|<k_{r}$ whenever $x_{n}, u_{n}$ is a minimizing sequence for $g_{r}(u \in S)$.

Let $\varepsilon_{r}=\sqrt{k_{r}}$. According to the variational principle of Ekeland, there are $x^{\prime} \in X, u^{\prime} \in S$ such that $\left\|x^{\prime}-z\right\| \leqslant \varepsilon_{r}$ and

$$
p_{r}(x, u)=g_{r}(x, u)+\varepsilon_{r}\left(\left\|x-x^{\prime}\right\|+\left\|u-u^{\prime}\right\|\right)
$$

attains a minimum on $B(z, c) \times S$ at $\left(x^{\prime}, u^{\prime}\right)$. (We consider $r$ sufficiently large to ensure $\varepsilon_{r}<c$.)

The function $p_{r}$ is obviously Lipschitz in $u$ with Lipschitz constant $L^{\prime}$ near $\left(x^{\prime}, u^{\prime}\right)$ which is equivalent to $r\left\|x^{\prime}-u^{\prime}\right\|($ as $r \rightarrow \infty)$. Then the function $q_{r}(x, u)=p_{r}(x, u)$ $+L_{r} \rho(u, S)$, where $L_{r}=L^{\prime}+(1 / r)$, attains an unconditional minimum at $\left(x^{\prime}, u^{\prime}\right)$ (i.e. without the restriction $u \in S$ ). It follows that

$$
d^{-} q_{r}\left(\left(x^{\prime}, u^{\prime}\right) ;(h, v)\right) \geqslant 0, \quad \forall h \in X, \forall v \in X .
$$

Let $x_{r}^{*}$ be the derivative of $\|\cdot\|$ at $x^{\prime}-u^{\prime}$ if $x^{\prime} \neq u^{\prime}$ or an arbitrary element of the unit ball of $X^{*}$ otherwise. Then the inequality above implies

$$
d^{-} f\left(x^{\prime} ; h\right)+r\left\|x^{\prime}-u^{\prime}\right\|\left\langle x_{r}^{*}, h-v\right\rangle+\varepsilon_{r}(\|h\|+\|v\|)+L_{r} d^{-} \rho\left(u^{\prime}, S ; v\right)>0
$$

for all $h, v$.

Setting $h=0$ in this inequality, we have

$$
d^{-} \rho\left(u^{\prime}, S ; v\right)+\varepsilon_{r}\|v\| \geqslant\left(r / L_{r}\right)\left\|x^{\prime}-u^{\prime}\right\|\left\langle x_{r}^{*}, v\right\rangle,
$$

or in other words, $\left(r / L_{r}\right)\left\|x^{\prime}-u^{\prime}\right\| x_{r}^{*} \in \partial_{e_{r}}{ }^{-} \rho\left(u^{\prime}, S\right)$. Setting $v=0$, we have

$$
d^{-} f\left(x^{\prime} ; h\right)+\varepsilon_{r}\|h\| \geqslant-r\left\|x^{\prime}-u^{\prime}\right\|\left\langle x_{r}^{*}, h\right\rangle
$$

so that $r\left\|x^{\prime}-u^{\prime}\right\|\left\|x_{r}^{*}\right\| \leqslant L+\sqrt{L / 2 r}$ and $u_{r}^{*}=-r\left\|x^{\prime}-u^{\prime}\right\| x_{r}^{*} \in \partial_{e}-f\left(x^{\prime}\right)$.

If $r \rightarrow \infty$, then, obviously, $x^{\prime} \rightarrow z, u^{\prime} \rightarrow z, \varepsilon_{r} \rightarrow 0,\left(r / L_{r}\right)\left\|x^{\prime}-u^{\prime}\right\| \rightarrow 1$. Taking if necessary a subsequence, we may assume that $r\left\|x^{\prime}-u^{\prime}\right\|$ converges to some $\lambda>0$. If now $x^{*}$ is a weak ${ }^{*}$ limit point of the sequence $\left\{x_{r}^{*}\right\}$, then $u^{*}=-\lambda x^{*}$ is a weak limit point of $\left\{u_{r}^{*}\right\}$ and $x^{*} \in \partial_{m} \rho(z, S)$ so that $-u^{*}=\lambda x^{*} \in N_{m}(S, z)$ and $u^{*} \in$ $\partial_{m} f(z)$ which completes the proof.

\section{Prederivatives and derivatives.}

9.1. Definition. Let $F$ be a mapping from a neighbourhood of $z \in X$ into $Y$ and $Q$ a homogeneous set-valued mapping from $X$ into $Y$. Consider the relations:

$$
\begin{gathered}
F(z+h)-F(z) \subset \mathbb{Q}(h)+r(h)\|h\| B_{Y}, \\
\mathbb{Q}(h) \subset \bigcup_{0<t<1} t^{-1}(F(z+t h)-F(z))+r(h)\|h\| B_{Y} .
\end{gathered}
$$

We shall say that $\mathcal{Q}$ is:

an outer prederivative (or an outer Fréchet prederivative) of $F$ at $z$ if (1) holds with $r(h) \rightarrow 0$ if $\|h\| \rightarrow 0$; 
a weak outer prederivative (or an outer Gâteaux prederivative) if (1) holds with $r(h) \rightarrow 0$ when $h$ tends to zero along any finite-dimensional subspace of $X$;

an inner prederivative (or an inner Fréchet prederivative) of $F$ at $z$ if (2) holds with $r(h) \rightarrow 0$ if $\|h\| \rightarrow 0$

a weak inner prederivative (or an inner Gâteaux prederivative) if (2) holds with $r(h) \rightarrow 0$ when $h$ tends to zero along any finite-dimensional subspace of $X$.

A homogeneous mapping which is both an outer and an inner prederivative (resp. a weak outer and a weak inner prederivative) will be called a derivative or a Fréchet derivative (resp. a weak derivative or a Gâteaux derivative) of $F$ at $z$.

A homogeneous mapping $Q$ will be called a strict prederivative of $F$ at $z$ if

$$
F(x+h)-F(x) \in \mathbb{Q}(h)+r(x, h)\|h\| B_{Y}
$$

where $r(x, h) \rightarrow 0$ if $\|x-z\| \rightarrow 0,\|h\| \rightarrow 0$.

9.2. Proposition. If $\mathcal{Q}$ is an outer (or a weak outer) prederivative and $\mathscr{B}$ is an inner (or a weak inner) prederivative, then $\mathscr{B}(h) \subset \mathbb{Q}(h)$ for all $h$. Thus either derivative, if it exists, is uniquely defined. In particular, any derivative is a weak derivative.

Proof. Let $y \in \mathscr{B}(h)$. Then for any $\lambda>0$, there is $0<t \leqslant 1$ such that

$$
\begin{aligned}
\lambda y \in t^{-1} & (F(z+t \lambda h)-F(z))+\lambda r(\lambda h)\|h\| B_{Y} \\
& \subset t^{-1} \mathscr{Q}(t \lambda h)+2 \lambda r(\lambda h)\|h\| B_{Y} \\
& =\lambda \mathscr{Q}(h)+2 \lambda r(\lambda h)\|h\| B_{Y},
\end{aligned}
$$

so that $y \in \mathbb{Q}(h)+2 r(\lambda h)\|h\| B_{Y}$. This is true for any $\lambda>0$ and therefore $y \in$ $\mathbb{Q}(h)$.

9.3. Remark. Clearly, usual Fréchet or strict Fréchet derivatives (see [7]) are, respectively, derivatives and strict prederivatives in the sense just defined.

Let us give some more examples. Assume that we have a set $\mathfrak{A}$ of linear operators from $X$ into $Y$ such that for any $h \in X$ there is $A \in \mathfrak{A}$ such that $\| F(z+h)-F(z)$ $-A h\|\leqslant r(h)\| h \|$ (or for any $x$ and any $h$ there is $A \in \mathfrak{A}$ such that $\| F(x+h)-$ $F(x)-A h\|\leqslant r(x, h)\| h \|)$. Then the set-valued mapping $\mathcal{Q}(h)=\overline{\bigcup_{A \in \mathcal{A}} A h}$ is an outer prederivative (strict prederivative).

Derivate containers of Warga [56], [60] and screens and fans of Halkin [18], [19] belong to this class. One can expect that derivatives defined by way of operators have some additional good properties. Therefore the question when such prederivatives exist seems to be important. We shall discuss it in the next section.

Various concepts of derivatives can be obtained using one or another tangent cone to the graph of the mapping. Thus the set-valued mapping $\mathbb{Q}(h)=\{y \in$ $Y \mid(h, y) \in K_{\varepsilon}($ Graph $\left.F,(z, F(z)))\right\}$ is an outer prederivative of $F$ at $z$.

More important is the contingent derivative studied by Aubin [3]. It is defined in a similar way to the tangent cone:

$$
D^{-} F(z)(h)=\{y \in Y \mid(h, y) \in T(\operatorname{Graph} F,(z, F(z)))\} .
$$

9.4. Proposition. If the weak derivative of $F$ at $z$ exists, it coincides with $D^{-} F(z)$. In particular, if $\operatorname{dim} Y<\infty$ and $F$ is Lipschitz, then $D^{-} F(z)$ is the weak derivative of $F$ at $z$. If in addition $\operatorname{dim} X<\infty$ then $D^{-} F(z)$ is the derivative of $F$ at $z$. 
Proof. Let $\mathcal{Q}(h)$ be a weak inner prederivative of $F$ at $z$. Substituting $\varepsilon h /\|h\|$ for $h$ in (2) and setting $\lambda=\varepsilon t /\|h\|$, we get

$$
\mathbb{Q}(h) \subset \bigcup_{0<\lambda\|h\|<\varepsilon} \lambda^{-1}(F(z+\lambda h)-F(z))+r(\varepsilon h /\|h\|)\|h\| B_{Y} .
$$

Since this is true for any $\varepsilon>0$, the inclusion $\mathscr{Q}(h) \subset D^{-} F(z)(h)$ follows from 8.3.

On the other hand, as follows from $(1), D^{-} F(z)(h) \subset \mathbb{Q}(h)$ whenever $\mathbb{Q}$ is an outer prederivative. Thus if the derivative exists, it must coincide with $D^{-} F(z)(h)$ by virtue of 9.2 .

If both $X, Y$ are finite-dimensional and $F$ is Lipschitz, then 8.5 shows that $D^{-} F(z)$ is an outer prederivative, hence the derivative of $F$ at $z$. (Since $F$ is Lipschitz, the graph of the tangent cone to the graph of $F$ cannot contain any "vertical" vectors.) Finally, if only $Y$ is finite-dimensional and $F$ is Lipschitz, then the restriction of $D^{-} F(z)$ to every finite-dimensional subspace coincides with the contingent derivative of the restriction of $F$ to the corresponding linear manifold at $z$.

9.5. REMARK. If $\operatorname{dim} Y=\infty$, the contingent derivative can be trivial even for Lipschitz mappings (we again refer to 8.6). If this happens, the only "derivatives" we can work with are outer or strict prederivatives. The theorem below introduces two classes of objects which will be the focus of our attention in the rest of the paper.

9.6. THEOREM. Let $F$ be a continuous mapping from a neighbourhood of $z \in X$ into $Y$. For any $y^{*} \in Y^{*}$, set $f_{y^{*}}(x)=\left\langle y^{*}, F(x)\right\rangle$. Then, for any $\varepsilon>0$, the function

$$
\left(y^{*}, h\right) \rightarrow F_{\varepsilon}^{0}\left(z ; y^{*}, h\right)=d_{e}^{0} f_{y^{*}}(z ; h)
$$

is the support function of an odd fan $D_{\varepsilon}^{0} F(z)$ which is a strict prederivative of $F$ at $z$, and the function

$$
\left(y^{*}, h\right) \rightarrow F_{e}^{+}\left(z ; y^{*}, h\right)=d_{e}^{+} f_{y^{*}}(z ; h)
$$

is the support function of a prefan $D_{e}^{+} F(z)$ which is an outer prederivative of $F$ at $z$.

Proof. The function $F_{\varepsilon}^{0}\left(z ; y^{*}, h\right)$ is everywhere more than $-\infty$ and it is sublinear and weak* 1.s.c. in $y^{*}$ (as the upper bound of a collection of linear continuous functions). According to 8.2, it is also sublinear in $h$. Thus it is bisublinear and, by 1.4, the support function of a fan which we denote by $D_{\varepsilon}^{0} F(z)$. As follows from 8.1 , $\left\langle y^{*}, F(x+h)-F(x)\right\rangle \leqslant F_{e}^{0}\left(z ; y^{*}, h\right)$ if $\|x-z\|<\varepsilon,\|x+h-z\|<\varepsilon$; hence $D_{\varepsilon}^{0} F(z)$ is a strict prederivative of $F$ at $z$. Finally, the equality

$$
\left\langle y^{*}, F(x+h)-F(x)\right\rangle=\left\langle-y^{*}, F(u-h)-F(u)\right\rangle
$$

for $u=x+h$ shows that $D_{\varepsilon}^{0} F(z)$ is an odd fan (see 1.7).

The second part can be proved similarly.

9.7. Definition. The prederivatives introduced above will be called the strict $\varepsilon$-prederivative and the outer $\varepsilon$-prederivative of $F$ at $z$. Both are always well defined (i.e. the domain of each of them is all of $X$ ). Observe that the strict $\varepsilon$-prederivative is bounded if and only if $F$ is Lipschitz in the $\varepsilon$-neighbourhood of $z$. 
Support functions of both prederivatives decrease as $\varepsilon \downarrow 0$ and converge respectively to

$$
F^{0}\left(z ; y^{*}, h\right)=d^{0} f_{y^{*}}(z ; h) \text { and } F^{+}\left(z ; y^{*}, h\right)=d^{+} f_{y^{*}}(z ; h)
$$

which can fail to be weak* 1.s.c. in $y^{*}$. But if they are, then the corresponding fan and prefan will be denoted by $D^{0} F(z)$ and $D^{+} F(z)$. The first of them will be called the upper derivative of $F$ at $z$.

9.8. Proposition. Let $\mathcal{Q}$ be a strict (resp. an outer) prederivative of $F$ at $z$. Then for any $\delta>0$, there is $\varepsilon>0$ such that

$$
D_{\varepsilon}^{0} F(z)(h) \subset \operatorname{conv} \mathbb{Q}(h)+\delta\|h\| B_{Y}
$$

(resp. $\left.D_{e}^{+} F(z)(h) \subset \operatorname{conv} \mathcal{Q}(h)+\delta\|h\| B_{Y}\right)$ for all $h$, and $\left(\right.$ if $D^{0} F(z)$ and $D^{+} F(z)$ exist)

$$
D^{+} F(z)(h) \subset D^{0} F(z)(h) \subset \overline{\operatorname{conv}} \mathbb{Q}(h), \quad \forall h .
$$

Proof. This follows immediately from 8.1, 9.1, 9.6.

9.9. REMARK. The proposition above means that any strict prederivative is almost a fan up to the convex closure of their values. Thus, among convex-valued strict prederivatives only fans should be considered. This is an important conclusion because the analytical virtues of fans enable us to extend all the propositions of the smooth differential calculus involving strict derivatives to nonsmooth mappings with fans as substitutes for strict derivatives.

However, it would be wrong to conclude that non-convex-valued strict prederivatives are unnecessary. We refer to [60] for an example of a mapping $F$ whose derivate container (which is a nonconvex strict prederivative) satisfies conditions guaranteeing that $F$ is surjective whereas any $D^{0} F(z)$ fails to satisfy such a condition.

9.10. Proposition. Let there exist a strict (resp. an outer) prederivative $\mathbb{Q}$ of $F$ at $z$ with weakly compact values. Then $D^{0} F(z)$ (resp. $\left.D^{+} F(z)\right)$ exists. If in addition the values of $Q$ are norm compact and $F$ is Lipschitz, then $D_{e}^{0} F(z)$ converges to $D^{0} F(z)$ in the topology of compact convergence.

Proof. If the values of $\mathcal{Q}$ are weakly compact, then $s_{\mathscr{Q}}\left(y^{*}, x\right)$ is continuous in $y^{*}$ in the Mackey topology $\tau\left(Y^{*}, Y\right)$. In view of $9.9, F^{0}\left(z ; y^{*}, h\right)$ is also continuous in this topology and hence weak* 1. s.c. in $y^{*}$.

If $\mathcal{Q}$ is norm-compact-valued and $F$ is Lipschitz, then $D_{\varepsilon}^{0} F(z) \in \mathscr{F}(X, Y)$ and 5.8 along with the first inclusion in 9.8 imply compact convergence of $D_{e}^{0} F(z)$ to $D^{0} F(z)$.

9.11. Corollary. If $X$ and $Y$ are finite-dimensional and $F$ is Lipschitz, then $D^{0} F(z)$ is a strict prederivative of $F$ at $z$.

Proof. In this case compact and bounded convergences coincide and, as one can easily see, the collection of bounded fans which are strict prederivatives is closed under bounded convergence (not only in finite-dimensional spaces). 
9.12. Definition. If $F$ is Lipschitz, then the mapping $h \rightarrow F^{0}\left(z ; y^{*}, h\right)$ is norm continuous; hence the (bounded) fan

$$
D_{c}^{*} F(z)\left(y^{*}\right)=\left\{x^{*} \in X^{*}\left|F^{0}\left(z ; y^{*}, h\right)\right\rangle\left\langle x^{*}, h\right\rangle, \forall h \in X\right\}
$$

from $Y^{*}$ into $X^{*}$ is well defined. It is clear that $D_{c}^{*} F(z)=\left(D^{0} F(z)\right)^{*}$ if the latter exists and, on the other hand,

$$
D_{c}^{*} F(z)\left(y^{*}\right)=\partial_{c} f_{y^{*}}(z), \quad \forall y^{*} \in Y^{*} .
$$

We shall call $D_{c}^{*} F(z)$ the $C$-coderivative of $F$ at $z$.

The last formula suggests one more definition. The set-valued mapping

$$
D_{m}^{*} F(z)\left(y^{*}\right)=\partial_{m} f_{y^{*}}(z)
$$

(obviously homogeneous) will be called the $M$-coderivative of $F$ at $z$. It follows from 8.9 that $D_{m}^{*} F(z)\left(y^{*}\right) \subset D_{c}^{*} F(z)\left(y^{*}\right)$.

Observe that both definitions can be applied to non-Lipschitz mappings but there is no guarantee that they will be well defined.

9.13. REMARK. As follows from definitions, outer and strict prederivatives withstand scalar multiplication and summation which means, say, that the sum of prederivatives is a prederivative of a sum. It is also easy to verify that the composition of bounded outer or strict prederivatives is a prederivative (of the corresponding type) of the composition of the mappings involved.

For upper derivatives, we obviously have $(F+G)^{0}\left(z ; y^{*}, h\right)<F^{0}\left(z ; y^{*}, h\right)+$ $G^{0}\left(z ; y^{*}, h\right)$ so that $D^{0}(F+G)(z)(h) \subset D^{0} F(z)(h)+D^{0} G(z)(h)$ (if they exist) and $D_{c}^{*}(F+G)(z)\left(y^{*}\right) \subset D_{c}^{*} F(z)\left(y^{*}\right)+D_{c}^{*} G(z)\left(y^{*}\right)$. The situation with the composition operation is more complicated.

9.14. Proposition. Let $F: X \rightarrow Y$ and $G: Y \rightarrow W$ be Lipschitz about $z \in X$ and $v=F(z)$ respectively. If either $F$ has a strict prederivative at $z$ with norm compact values or the adjoint to a fan which is a strict prederivative of $G$ at $v$ is norm-compact-valued, then

$$
D_{c}^{*}(G \circ F)(z)\left(w^{*}\right) \subset\left(D_{c}^{*} F(z) \circ D_{c}^{*} G(v)\right)\left(w^{*}\right), \quad \forall w^{*} \in W^{*} .
$$

Proof. We only need to show that

$$
(G \circ F)^{0}\left(z ; w^{*}, h\right) \leqslant \sup \left\{G^{0}\left(v ; w^{*}, y\right) \mid y \in D^{0} F(z)(h)\right\}
$$

in the first case (in view of $9.10, D^{\circ} F(z)$ does exist) or

$$
(G \circ F)^{0}\left(z ; w^{*}, h\right) \leqslant \sup \left\{F^{0}\left(z ; y^{*}, h\right) \mid y^{*} \in D_{c}^{*} G(v)\left(w^{*}\right)\right\}
$$

(cf. 3.2, 3.3). We shall consider only the first case. The second can be treated similarly.

Assume that $F$ has a strict prederivative at $z$ with norm compact values. For any $\varepsilon>0, \delta>0, D_{\delta}^{0} G(v) \circ D_{\varepsilon}^{0} F(z)$ is a strict prederivative of $G \circ F$ at $z$; hence (see 9.8)

$$
(G \circ F)^{0}\left(z ; w^{*}, h\right)<\sup \left\{G_{\delta}^{0}\left(v ; w^{*}, y\right) \mid y \in D_{e}^{0} F(z)(h)\right\}
$$


According to $9.10, D_{\varepsilon}^{0} F(z)$ converges pointwise to $D^{0} F(z)$. Since the values of the latter are norm compact (thanks to 9.8), we conclude that, for any $h$,

$$
\lim _{\varepsilon \downarrow 0} \sup \left\{G_{\delta}^{0}\left(v ; w^{*}, y\right) \mid y \in D_{\varepsilon}^{0} F(z)(h)\right\}=\sup \left\{G_{\delta}^{0}\left(v ; w^{*}, y\right) \mid y \in D^{0} f(z)(h)\right\} .
$$

On the other hand, for any fixed $w^{*}$, the functions $G_{\delta}^{0}\left(v ; w^{*}, \cdot\right)$ are convex and continuous and converge to $G^{0}\left(v ; w^{*}, \cdot\right)$ as $\delta \downarrow 0$. Since pointwise convergence of continuous convex functions implies uniform convergence on every compact set, the result follows.

10. Mean value theorem and the 1-extension property.

10.1 Proposition (Mean Value Theorem). Let $F: X \rightarrow Y$ be defined and Lipschitz in a neighbourhood of the line segment $[z, z+h]$. Then, for any $y^{*} \in Y^{*}$,

$$
\left\langle y^{*}, F(z+h)-F(z)\right\rangle\left\langle\int_{0}^{1} F^{+}\left(z+t h ; y^{*}, h\right) d t .\right.
$$

In particular, if $D^{+} F(x)$ exists at every point of the line segment, then

$$
F(z+h)-F(z) \in \int_{0}^{1} D^{+} F(z+t h)(h) d t .
$$

(The integral in the last formula is understood as usual as the collection of integrals of all of the measurable selections of the set-valued mapping $t \rightarrow$ $\left.D^{+} F(z+t h)(h).\right)$

Proof. The first formula is an immediate corollary of the Radon-Nikodym theorem because

$$
(d / d t)\left\langle y^{*}, F(z+t h)\right\rangle\left\langle F^{+}\left(z+t h ; y^{*}, h\right)\right.
$$

and the function on the right is obviously measurable in $t$. The second formula follows directly from well-known formulas for subdifferentials of convex functions [31]. (Observe that $F^{+}$is norm continuous in $y^{*}$ since $F$ is Lipschitz.)

10.2. Corollary. Let $F: X \rightarrow Y$ be Lipschitz in a neighbourhood of $z \in X$. Then

$$
F_{\varepsilon}^{0}\left(z ; y^{*}, h\right)=\sup _{\|x-z\|<\varepsilon} F^{+}\left(x ; y^{*}, h\right)=\sup _{\|x-z\|<\varepsilon} F^{0}\left(x ; y^{*}, h\right)
$$

and

$$
D_{\varepsilon}^{0} F(z)(h)=\bigvee_{\|x-z\|<\varepsilon} D^{+} F(x)(h)=\bigvee_{\|x-z\|<\varepsilon} D^{0} F(x)(h)
$$

if $D^{+} F(x)$ and $D^{0} F(x)$ exist for all $x,\|x-z\|<\varepsilon$.

Proof. As follows from 10.1, the quantity on the left in the first formula cannot be greater than those on the right. On the other hand, $F_{e}^{0}\left(z ; y^{*}, h\right) \geqslant F^{+}\left(x ; y^{*}, h\right)$ if $\|x-z\|<\varepsilon$.

10.3. Proposition. Let $F$ be the same as in 10.1. Then, for any $y^{*} \in Y^{*}$, there are $t \in[0,1]$ and $x^{*} \in D_{c}^{*} F(z+t h)\left(y^{*}\right)$ such that $\left\langle y^{*}, F(z+h)-F(z)\right\rangle=\left\langle x^{*}, h\right\rangle$.

Proof. This results from the corresponding mean value theorem for Lipschitz real-valued functions [36] (see also [20], [21]). 
We shall now turn to the problem that we mentioned before: in what situations can the local behaviour of a Lipschitz mapping be described by sets of linear operators?

10.4. THEOREM. Let $F$ be a Lipschitz mapping from a neighbourhood of $z \in X$ into $Y$. If $F$ has a strict prederivative at $z$ with weakly compact values, then $D^{0} F(z)$ has the 1-extension property.

Proof. With no loss of generality we can assume that $z=0$.

In view of Theorem 7.7, it is enough to show that the restriction of $D^{\circ} F(z)$ to any finite-dimensional subspace of $X$ has the 1-extension property. (Observe that $D^{\circ} F(z)$ exists thanks to 9.10 and it is an odd bounded fan with weakly compact values as follows from 9.6,9.8.) This, in turn, will be proved if we show that, given a finite-dimensional subspace $U \subset X$, for any $y^{*} \in Y^{*}, h \in U$, there is a linear selection $A$ of the restriction of $D^{0} F(z)$ to $U$ such that

$$
\left\langle y^{*}, A h\right\rangle=F^{0}\left(z ; y^{*}, h\right) \text {. }
$$

Indeed, let $S$ be the collection of all linear selections of the restriction of $D^{0} F(z)$ to $U$, and let $Q$ be a strict prederivative of $F$ at $z$ with weakly compact values. Then $S$ is convex and, in view of 9.8, $A \in S \Rightarrow A h \in D^{0} F(z)(h) \subset \mathbb{Q}(h), \forall h \in U$, which yields weak precompactness of $S$ in the topology of pointwise convergence associated with the weak topology of $Y[6$, Chapter 3, §3]. But $S$ is obviously closed in this topology (and moreover complete because the values of $\mathbb{Q}$ are weakly compact) so that, actually, $S$ is compact; hence all sets $\mathscr{B}(h)=\{y \in Y \mid y=A h$ for certain $A \in S$ \} are closed.

We have $\mathscr{B}(h) \subset D^{0} F(z)(h)$ and, in view of (1),

$$
\delta^{*}\left(y^{*}, \mathfrak{B}(h)\right)=F^{0}\left(z ; y^{*}, h\right)=\delta^{*}\left(y^{*}, D^{0} F(z)(h)\right)
$$

which implies the equality $\mathscr{B}(h)=D^{0} F(z)(h)$ because two closed convex sets coincide if their support functions are equal.

Now let $U$ be a finite dimensional subspace of $X$ and $W$ a complementary subspace so that any $x \in X$ can be uniquely represented as a sum $x=u+w$, $u \in U, w \in W$. Renorming $X$, if necessary, we can assume that $U$ is a Euclidean space in the induced norm. Let $d u$ denote the Lebesgue measure on $U$.

Choose a mollifier $f$ on $U$, that is to say, a continuously differentiable nonnegative function which vanishes outside the unit ball and satisfies $\int_{U} f(u) d u=1$. For any sufficiently small $\varepsilon>0$, let us define a mapping $F_{\varepsilon}$ from $X$ into $Y$ by

$$
\begin{aligned}
F_{e}(x) & =\varepsilon^{-1} \int_{U} F(x-u) f(u / \varepsilon) d u \\
& =\varepsilon^{-1} \int_{U} F(\xi+w) f((u-\xi) / \varepsilon) d \xi=F_{e}(u, w)
\end{aligned}
$$

Clearly, $F$ is well defined and continuously differentiable in $u$ in a neighbourhood of the origin (recall that $z=0$ ). Let us denote by $F_{e}^{\prime}$ the derivative of $F_{e}$ in $u$. Then, 
given $y^{*} \in Y^{*}, h \in U$, we have

$$
\begin{aligned}
\left\langle y^{*}, F_{\varepsilon}^{\prime}(x) h\right\rangle & =\lim _{t \downarrow 0} \varepsilon^{-1} \int_{U} t^{-1}\left\langle y^{*}, F(x-u+t h)-F(x-u)\right\rangle f(u / \varepsilon) d u \\
& \leqslant \varepsilon^{-1} \int_{U} F^{0}\left(x-u ; y^{*}, h\right) f(u / \varepsilon) d u \\
& \leqslant \sup _{u \in \varepsilon B_{U}} F^{0}\left(x-u ; y^{*}, h\right) .
\end{aligned}
$$

We also have $F(x+h)-F(x) \in \mathbb{Q}(h)+r(x, h)\|h\| B_{Y}$ so that

$$
\begin{aligned}
\left\langle y^{*}, F_{\varepsilon}^{\prime}(x) h\right\rangle & \leqslant \lim _{t \downarrow 0} \varepsilon^{-1} \int_{U}\left(s_{\mathbb{Q}}\left(y^{*}, h\right)+r(x-u, t h)\left\|y^{*}\right\|\|h\|\right) f(u / \varepsilon) d u, \\
& \left\langle s_{\mathbb{Q}}\left(y^{*}, h\right)+r_{\varepsilon}(x)\left\|y^{*}\right\|\|h\|,\right.
\end{aligned}
$$

where $r_{\varepsilon}(x)=\lim \sup _{\|h\| \rightarrow 0} \sup _{\|u\|<\varepsilon} r(x-u, h) \rightarrow 0$ if $\|x\| \rightarrow 0, \varepsilon \rightarrow 0$.

It follows that, for any $h \in U$,

$$
F_{e}^{\prime}(x) h \in \mathbb{Q}(h)+r_{e}(x)\|h\| B_{Y} .
$$

Now fix $v^{*} \in Y^{*}, e \in U$, and let sequences $x_{n} \rightarrow 0, t_{n} \downarrow 0$ be such that

$$
t_{n}^{-1}\left\langle v^{*}, F_{n}\left(x_{n}+t_{n} e\right)-F\left(x_{n}\right)\right\rangle \rightarrow F^{0}\left(z ; v^{*}, e\right) \text {. }
$$

Take $\varepsilon_{n} \downarrow 0$ and $0 \leqslant \lambda_{n} \leqslant 1$ in such a way that

$$
\left\langle v^{*}, F_{n}\left(x_{n}+t_{n} e\right)-F\left(x_{n}\right)\right\rangle=t_{n}\left\langle v^{*}, A_{n} e\right\rangle \text {, }
$$

where $F_{n}=F_{e_{n}}, A_{n}=F_{n}^{\prime}\left(x_{n}+\lambda_{n} t_{n} e\right)$.

Then (3) implies that, for any $h \in U, A_{n} h \in \mathbb{Q}(h)+r_{n}\|h\| B_{Y}$, where $r_{n}=$ $r_{e_{n}}\left(x_{n}+t_{n} e\right) \rightarrow 0$ as $n \rightarrow \infty$. It follows that the sequence $\left\{A_{n}\right\}$ is uniformly bounded, hence equicontinuous, and (since $Q(h)$ is weakly precompact for any $h$ ) for each $h \in U$ the sequence $\left\{A_{n} h\right\}$ is weakly precompact.

This implies that $\left\{A_{n}\right\}$ is precompact in the topology of pointwise convergence associated with the weak topology of $Y$. Let $A$ be a limit point of the sequence. Then

$$
\liminf _{n \rightarrow \infty}\left\langle y^{*}, A_{n} h\right\rangle\left\langle\langle y ^ { * } , A h \rangle \left\langle\lim _{n \rightarrow \infty}\left\langle y^{*}, A_{n} h\right\rangle\right.\right.
$$

and (2) implies

$$
\begin{aligned}
\left\langle y^{*}, A h\right\rangle & \leqslant \limsup _{n \rightarrow \infty} \sup _{\|u\|<e_{n}} F^{0}\left(x_{n}+t_{n} e-u ; y^{*}, h\right) \\
& \left\langle F^{0}\left(z ; y^{*}, h\right), \quad \forall y^{*} \in Y^{*}, \forall h \in U\right.
\end{aligned}
$$

(because $F^{0}$ is u.s.c. in the first argument). This is the same as $A h \in D^{0} F(z)(h)$ for all $h \in U$ which means that $A$ is a linear selection of $D^{0} F(z)$ on $U$.

On the other hand, as follows from (4)-(6),

$$
\left\langle v^{*}, A e\right\rangle=F^{0}\left(z ; y^{*}, e\right) \text {. }
$$

This completes the proof.

10.5. Corollary. If for some $\varepsilon>0$ the $\varepsilon$-prederivative $D_{\varepsilon}^{0} F(z)$ has weakly compact values, then $D_{\delta}^{0} F(z)$ has the 1-extension property for any $\delta<\varepsilon$. 
Proof. This can be obtained from the theorem using 10.2 and compactness arguments similar to those in the proof of the theorem.

Observe that it would be wrong to assert that any strict prederivative majorized by $D_{\varepsilon}^{0} F(z)$ has the 1-extension property.

10.6. Corollary. If $Y$ is a reflexive space, then strict $\varepsilon$-prederivatives and upper derivatives of Lipschitz mappings into $Y$ have the 1-extension property.

10.7. Proposition. The $C$-coderivative $D_{c}^{*} F(z)$ of a mapping $F: X \rightarrow Y$ which is Lipschitz in a neighbourhood of $z$ has the 1-extension property.

Proof. Fix a finite collection $\left\{y_{1}^{*}, \ldots, y_{n}^{*}\right\}$ of elements of $Y^{*}$ and consider the mapping $f: X \rightarrow R^{n}$ defined by

$$
f(x)=\left(\left\langle y_{1}^{*}, F(x)\right\rangle, \ldots,\left\langle y_{n}^{*}, F(x)\right\rangle\right)
$$

which is obviously Lipschitz.

Then for any $\lambda=\left(\lambda_{1}, \ldots, \lambda_{n}\right) \in R^{n}, f^{0}(z ; \lambda, h)=F^{0}\left(z ; \Sigma_{i} \lambda_{i} y_{i}^{*}, h\right)$ which means that $D_{c}^{*} f(z)$ is the restriction of $D_{c}^{*} F(z)$ to the subspace spanned by $\left\{y_{1}^{*}, \ldots, y_{n}^{*}\right\}$.

According to Theorem $10.4, D^{0} f(z)$ has the 1-extension property; hence $D_{c}^{*} f(z)$ also has the 1-extension property. It remains to observe that the values of $D_{c}^{*} F(z)$ are weak* compact and apply Theorem 7.7.

10.8. Definition. Let both $X$ and $Y$ be finite-dimensional. Then any locally Lipschitz mapping from $X$ into $Y$ is almost everywhere differentiable. Let $F$ : $X \rightarrow Y$ be Lipschitz in a neighbourhood of $z$. The set

$$
\partial F(z)=\operatorname{conv} \lim _{x \rightarrow z} F^{\prime}(x)
$$

(the convex hull of all limits of sequences of derivatives $F^{\prime}\left(x_{n}\right)$, where $x_{n} \rightarrow z$ and $F$ is differentiable at every $x_{n}$ ) is called the generalized Jacobian of $F$ at $z$ [9], [12].

Clearly, $\partial F(z)$ is a bounded closed convex set of linear operators from $X$ into $Y$.

10.9. Proposition. Let $X$ and $Y$ be finite-dimensional and $F$ be a Lipschitz mapping from a neighbourhood of $z \in X$ into $Y$. Then the fan generated by $\partial F(z)$ coincides with $D^{0} F(z)$.

Proof. As follows from [8] and 8.9, for any $y^{*} \in Y^{*}$,

$$
D_{c}^{*} F(z)\left(y^{*}\right)=\overline{\operatorname{conv}} \lim _{\substack{x \rightarrow z \\ x \rightarrow G}} f_{y^{*}}^{\prime}(x)
$$

where $f_{y^{*}}(x)=\left\langle y^{*}, F(x)\right\rangle$ and $G \subset X$ is an arbitrary set whose complement has measure zero.

Let $G$ consist of all those points where $F$ is differentiable. Then $f_{y^{*}}^{\prime}(x)=$ $\left(F^{\prime}(x)\right)^{*}\left(y^{*}\right)$ for any $x \in G$; hence $D_{c}^{*} F(z)\left(y^{*}\right)=\left\{A^{*} y^{*} \mid A \in \partial F(z)\right\}$, which is the same as claimed.

It would probably be wrong to conclude that $\partial F(z)$ contains all linear selections of $D^{\circ} F(z)$. This is a likely explanation for the difficulties encountered in extending the chain rule using generalized Jacobians (cf. [11] and 9.14). 
11. Surjection theorems and applications. It is one of the central results of the classical differential calculus that a mapping $F: X \rightarrow Y$, strictly differentiable at $z$, maps neighbourhoods of $z$ onto neighbourhoods of $F(z)$, provided the derivative $F^{\prime}(z)$ is surjective (i.e. the image of $F^{\prime}(z)$ is all of $Y$ ). This section contains various extensions of this result to nonsmooth mappings and related results such as versions of Ljusternik's theorem, implicit function theorem and inverse mapping theorem. We refer to [9], [18], [19], [25], [38], [46], [56]-[60] for earlier results on the subject.

11.1. Definition. Let $F$ be a mapping from a neighbourhood of $z \in X$ into $Y$, and let $S \subset X$ contain $z$. The function

$$
t \rightarrow \omega(F, S, z)(t)=\sup \{r>0 \mid B(F(z), r) \subset F(B(z, t) \cap S)\}
$$

will be called the modulus of surjection of $F$ on $S$ at $z$. The quantity

$$
\gamma(F, S, z)=\liminf _{t \rightarrow 0} t^{-1} \omega(F, S, z)(t)
$$

will be called the constant of surjection of $F$ on $S$ at $z$.

11.2. LeMMA. Let $F: X \rightarrow Y$ be a continuous mapping defined in a neighbourhood of $z$, let $y \neq F(z)$, and let for any $n=1,2, \ldots$

$$
D_{n}=\left\{y^{*} \in Y^{*} \mid\left\|y^{*}\right\|\left\langle 1,\left\langle y^{*}, y-F(z)\right\rangle>(1-(1 / n))\|y-F(z)\|\right\} .\right.
$$

Let $f(x)=\|y-F(x)\|$. Then for any $\varepsilon>0$,

$$
d^{+} f(z ; h)<\lim _{n \rightarrow \infty} \sup _{y^{*} \in-D_{n}} F_{e}^{+}\left(z ; y^{*}, h\right), \quad \forall h .
$$

Proof. Fix $h \in X$. Then $F(z+t u) \rightarrow F(z)$ if $t \downarrow 0, u \rightarrow h$. Choose arbitrarily $y^{*}(t, u) \in Y^{*}$ such that $\left\|y^{*}(t, u)\right\|=1$ and

$$
\left\langle y^{*}(t, u), \quad y-F(z+t u)\right\rangle=\|y-F(z+t u)\| .
$$

Then $y^{*}(t, u) \in D_{n}$ if $t$ is sufficiently small and $u$ is sufficiently close to $h$. We have therefore

$$
\begin{aligned}
& d^{+} f(z ; h)=\limsup _{\substack{t \downarrow 0 \\
u \rightarrow h}} t^{-1}(\|y-F(z+t u)\|-\|y-F(z)\|) \\
& \left\langle\lim \sup t^{-1}\left\langle y^{*}(t, u), F(z)-F(z+t u)\right\rangle\right. \\
& \underset{u \rightarrow h}{t \downarrow 0} \\
& \leqslant \underset{\substack{t \downarrow 0 \\
u \rightarrow h}}{\lim \sup } \sup _{\substack{0<\tau\|w\|<\varepsilon \\
\|w-h\|<\varepsilon}} \tau^{-1}\left\langle-y^{*}(t, u), F(z+\tau w)-F(z)\right\rangle \\
& =\lim \sup F_{e}^{+}\left(z ;-y^{*}(t, u), h\right) \\
& \underset{u \rightarrow h}{t \downarrow 0} \\
& \leqslant \lim _{n \rightarrow \infty} \sup _{y^{*} \in-D_{n}} F_{e}^{+}\left(z ; y^{*}, h\right) \text {. }
\end{aligned}
$$

11.3. Remark. Observe that (since $y \neq F(z)$ ) every $D_{n}$ is a weak* closed convex subset of the unit ball of $Y^{*}$ such that $\left\|y^{*}\right\|>1-(1 / n)$ whenever $y^{*} \in D_{n}$. 
11.4. Lemma. Let $F: X \rightarrow Y$ be continuous in a neighbourhood of $z \in S$, let $t>0$ be such that $B(z, t)$ belongs to the domain of $F$, and let $y \notin F(B(z, t) \cap S)$. Set $k=\|y-F(z)\|, f(x)=\|y-F(x)\|$. Then for any $0<\tau<t$ there is $w \in S$ such that $\|z-w\| \leqslant \tau$ and the function

$$
g(x)=f(x)+(k / \tau)\|x-w\|
$$

attains at $w$ its minimum on $B(z, t) \cap S$.

Proof. We have $f(x)>0$ for all $x \in B(z, t) \cap S$ and $f(z)=k$. It remains to apply the variational principle of Ekeland [16].

11.5. TheOREM. Let $F$ be continuous near $z$. Then

$$
t^{-1} \omega(F, S, z)(t) \geqslant \inf _{\substack{\|x-z\|<t \\ x \in S}} \lim _{e \downarrow 0} \operatorname{sl}\left(D_{e}^{+} F(x), T(S, x)\right) .
$$

Proof. It follows from 11.4 that $d^{+} f(w ; h)+(k / \tau)\|h\|>0, \forall h \in T(S, w)$. In view of Lemma 11.2, this implies that

$$
\lim _{n \rightarrow \infty} \sup _{y^{*} \in-D_{n}} F_{e}^{+}\left(w ; y^{*}, h\right)+(k / \tau)\|h\| \geqslant 0, \quad \forall h \in T(S, w),
$$

where $D_{n}=\left\{y^{*} \mid\left\|y^{*}\right\| \leqslant 1,\left\langle y^{*}, y-F(w)\right\rangle>(1-(1 / n))\|y-F(w)\|\right\}$. Since $y \neq F(w)$, it follows from 11.3 that $\left\{D_{n}\right\} \in \mathscr{D}$ (see 5.9) and we have from (1)

$$
-\lim _{\varepsilon \downarrow 0} \operatorname{sl}\left(D_{e}^{+} F(w), T(S, w)\right)+(k / \tau)>0
$$

or

$$
\begin{aligned}
\tau^{-1}\|y-F(w)\| & =k / \tau \geqslant \lim _{\varepsilon \downarrow 0} \operatorname{sl}\left(D_{e}^{+} F(w), T(S, w)\right) \\
& \geqslant \inf _{\substack{\|x-z\|<t \\
x \in S}} \lim _{\varepsilon \downarrow 0} \operatorname{sl}\left(D_{e}^{+} F(x), T(S, x)\right)=a .
\end{aligned}
$$

Since $\tau<t$ is arbitrary, it follows that any $y \in Y$ such that $\|y-F(z)\|<t a$ belongs to $F(B(z, \tau) \cap S)$ for certain $\tau$.

11.6. REMARK. As follows from $5.15, \operatorname{sl}\left(D_{e}{ }^{+} F(x), T(S, x)\right)$ can be replaced by $C\left(D_{e}{ }^{+} F(x), T(S, x)\right)$ if $F$ is Fréchet differentiable at every $x$ and $S$ is convex.

Let $\mathbb{Q}$ be a strict prederivative of $F$ at $z$, i.e. $F(x+h)-F(x) \in \mathbb{Q}(h)+$ $r(x, h)\|h\| B_{Y}$. We set

$$
r(\lambda)=\lim _{\varepsilon \downarrow 0} \sup \{r(x, h) \mid\|x-z\|<\lambda,\|h\|<\delta\} .
$$

11.7. ThEOREM. Assume that $F$ is Lipschitz in a neighbourhood of $z$ with constant $L$. Then the ratio $\omega(F, S, z)(t) / t$ is not less than any of the following quantities:

$$
a_{1}(t)=\inf _{\substack{\|x-z\|<t \\ x \in S}} \sup _{\delta>0} \lim _{\varepsilon \downarrow 0} \frac{\operatorname{sl}\left(D_{e}^{+} F(x), T_{\delta}(S, x)\right)-L \delta}{1+\delta},
$$

(here we set $T_{0}(S, x)=T(S, x)$ ),

$$
a_{2}(t)=\inf _{\substack{\|x-z\|<t \\ x \in S}} \sup _{\delta>0} \frac{\mathrm{sl}\left(\mathcal{Q}, T_{\delta}(S, x)\right)-L \delta-r(\|x-z\|+t)}{1+\delta}
$$


for any bounded fan $\mathbb{Q}$ which is a strict prederivative of $F$ at $z$;

$$
a_{3}(t)=\sup _{\delta>0} \frac{\operatorname{Sl}\left(D_{t}^{0} F(z), T(S, z)\right)-L \delta-\mu(t)}{1+\delta},
$$

where $\mu(t)=\sup \left\{\left|d^{-} \rho(z, S ; h)-d^{-} \rho(x, S ; h)\right| \mid\|x-z\|<t,\|h\|<1\right\}$.

Proof. Let $y, k, w$ be as above. The function $g(x)$ is then Lipschitz with constant not exceeding $L+(k / \tau)$ and (since $g$ attains its minimum on $B(z, t) \cap S$ at $w$ ),

$$
q(x)=g(x)+(L+(k / \tau)) \rho(x, S)>q(w)=g(w)
$$

for all $x \in B(z, t)$. Since $w \in$ int $B(z, t)$, it follows that

$$
0 \leqslant d^{-} q(w ; h) \leqslant d^{+} g(w ; h)+(L+(k / \tau)) d^{-} \rho(w, S ; h), \quad \forall h \in X
$$

and, as in 11.4, we deduce from here that for any $\varepsilon>0, \delta>0$

$$
\lim _{n \rightarrow \infty} \sup _{y^{*} \in-D_{n}} F_{\varepsilon}^{+}\left(w ; y^{*}, h\right)+(k / \tau)\|h\|+(L+(k / \tau)) \delta\|h\|>0
$$

for any $h \in T_{\delta}(S, w)$. It follows that

$$
k / \tau \geqslant \operatorname{sl}\left(D_{e}^{+} F(w), T_{\delta}(S, w)\right)-(L+(k / \tau)) \delta
$$

or

$$
k / \tau \geqslant \frac{\operatorname{sl}\left(D_{e}^{+} F(w), T_{\delta}(S, w)\right)-L \delta}{1+\delta}
$$

and the same arguments as in 11.4 give the inequality $\omega(F, S, z)(t) / t>a_{1}(t)$.

To prove that $\omega(F, S, z)(t) \geqslant t a_{2}(t)$, we note that, for any $\lambda>0, F(x+h)-$ $F(x) \in \mathbb{G}_{\lambda}(h)=\mathbb{Q}(h)+r(\lambda)\|h\| B_{Y}$ if $\|x-z\|\left\langle\lambda\right.$; hence $\left.\operatorname{sl}\left(D_{e}^{+} F(x), K\right)\right\rangle$ $\operatorname{sl}\left(Q_{\lambda}, K\right) \geqslant \operatorname{sl}(Q, K)-r(\lambda)$ for any cone $K$ (see 5.11).

Let us prove finally that $\omega(F, S, z)(t) \geqslant t a_{3}(t)$. For this purpose it suffices to show that

$$
\omega(F, S, z)(t) / t \geqslant \sup _{\delta>0} \frac{\operatorname{sl}\left(D_{t}^{0} F(z), T_{\delta}(S, z)\right)-L \delta-\mu(t)}{1+\delta}
$$

and then apply this (for any fixed $t$ ) to a sequence of equivalent norms in $Y^{*}$ converging to $\|\cdot\|$ and such that

$$
\operatorname{sl}\left(D_{t}^{0} F(z), T_{\delta}(S, z),|\cdot|_{n}\right) \rightarrow \operatorname{Sl}\left(D_{t}^{0}, F(z), T_{\delta}(S, z)\right) .
$$

If $\varepsilon+\tau<t$, then $F_{\varepsilon}^{+}\left(w ; y^{*}, h\right) \leqslant F_{t}^{0}\left(z ; y^{*}, h\right)$ and we have from (2), (3)

$$
\lim _{n \rightarrow \infty} \sup _{y^{*} \in-D_{n}} F_{t}^{0}\left(z ; y^{*}, h\right)+(k / \tau)\|h\|+(L+(k / \tau))\|h\|-\mu(t)\|h\| \geqslant 0
$$

whenever $h \in T_{\delta}(S, z)$. The rest of the proof is the same.

11.8. REMARK. If there is an equivalent locally uniformly convex norm in $Y^{*}$ or if there is an equivalent strictly convex norm and $D_{e}^{0} F(x)$ are norm-compact-valued, then it is possible to replace slopes sl and $\mathrm{Sl}$ by corresponding Banach constants (using 5.19). In general, 5.14 allows us to write other, rougher but more easily computable, estimations for moduli of surjection involving Banach constants. 
11.9. ThEOREM. Let $F$ be Lipschitz in a neighbourhood of $z$. Then

$$
\omega(F, S, z)(t) / t>\inf _{\substack{\|x-z\|<<\\ x \in S}} \lim _{\varepsilon \rightarrow 0} b(\varepsilon, x),
$$

where

$$
b(\varepsilon, x)=\mathrm{sl}^{*}\left(\left(D_{e}^{0} F(x)\right)^{*}, N_{c}(S, x)\right)
$$

in the general case;

$$
b(\varepsilon, x)=\mathrm{sl}^{*}\left(\left(D_{\varepsilon}^{0} F(x)\right) *, N_{m}(S, x)\right),
$$

if there is a Gâteaux differentiable equivalent norm in $X$;

$$
b(\varepsilon, x)=b(x)=C^{*}\left(D_{c}^{*} F(x), N_{c}(x)\right),
$$

if there is an equivalent locally uniformly convex norm in $Y^{*}$;

$$
b(\varepsilon, x)=b(x)=C^{*}\left(D_{m}^{*} F(x), N_{m}(S, x)\right)
$$

if there are both an equivalent Gâteaux differentiable norm in $X$ and an equivalent locally uniformly convex norm in $Y^{*}$.

Proof. We start as in 11.7. It follows that

$$
0 \in \partial_{c} g(w)+(L+(k / \tau)) \partial_{c} \rho(w, S) .
$$

Lemma 11.2 and Corollary 10.2 imply that

$$
d^{0} f(w ; h)<\lim _{n \rightarrow \infty} \sup _{y^{*} \in-D_{n}} F^{0}\left(w ; y^{*}, h\right)
$$

so that

$$
\begin{aligned}
\partial_{c} f(w) & \subset \bigcap_{n} \overline{\operatorname{conv}} \bigcup_{y^{*} \in-D_{n}}\left(D_{\varepsilon}^{0} F(w)\right)^{*}\left(y^{*}\right) \\
& =\bigcap_{n} W\left(\left(D_{e}^{0} F(w)\right)^{*},-D_{n}\right)
\end{aligned}
$$

(see 5.10); hence we have from (4)

$$
\begin{aligned}
0 & =\inf \left\{\left\|x^{*}+u^{*}\right\| \mid x^{*} \in \partial_{c} g(w), u^{*} \in N_{c}(S, w)\right\} \\
& =\inf \left\{\left\|x^{*}+u^{*}\right\| \mid x^{*} \in \partial_{c} f(w)+(k / \tau) B_{Y^{*}}, u^{*} \in N_{c}(S, w)\right\} \\
& >\inf \left\{\left\|x^{*}+u^{*}\right\| \mid x^{*} \in \partial_{c} f(w), u^{*} \in N_{c}(S, w)\right\}-(k / \tau)
\end{aligned}
$$

which, along with (5), yields

$$
k / \tau>\mathrm{sl}^{*}\left(\left(D^{0} F(w)\right)^{*}, N_{c}(S, w)\right) .
$$

If $X$ has an equivalent Gâteaux differentiable norm, then we can assume that $\|\cdot\|$ is such a norm (because $\mathbf{s l}^{*}$ depends continuously on the norm in $X$ ). Using 8.9, 8.11, we write instead of (4):

$$
0 \in \partial_{m} g(w)+N_{m}(S, w) \subset \partial_{c} g(w)+N_{m}(S, w)
$$

from which we conclude as above that

$$
k / \tau>\mathrm{sl}^{*}\left(\left(D_{e}^{0} F(w)\right)^{*}, N_{m}(S, w)\right) .
$$


Assume now that the norm in $Y^{*}$ is locally uniformly convex. Then we have, as in $11.2, f(x+t h)-f(x) \leqslant\left\langle y^{*}(t, x), F(x+t h)-F(x)\right\rangle$, where $\left\langle-y^{*}(t, x), y-\right.$ $F(x+t h)\rangle=\|y-F(x+t h)\|$. Since $F(x+t h) \rightarrow F(w)$ if $t \rightarrow 0$ and $x \rightarrow w$ and $y \neq F(w)$, it follows that $y^{*}(t, x)$ norm converges to the unique $y^{*}$ such that $\left\langle-y^{*}, y-F(w)\right\rangle=\|y-F(w)\|$. Therefore

$$
\begin{gathered}
d^{0} f(w ; h) \leqslant d^{0} f_{y^{*}}(w ; h), \\
d^{-} f(w ; h) \leqslant d^{-} f_{y^{*}}(w ; h),
\end{gathered}
$$

where, as above, $f(x)=\|y-F(x)\|$ and $f_{y^{*}}(x)=\left\langle y^{*}, F(x)\right\rangle$.

It follows from (9) that $\partial_{c} f(w) \subset D_{c}^{*}(w)\left(y^{*}\right)$ which, together with (4), yields

$$
\begin{aligned}
0 & =\inf \left\{\left\|x^{*}+u^{*}\right\| \mid x^{*} \in D_{c}^{*} F(w)\left(y^{*}\right)+(k / \tau) B_{Y^{*}}, u^{*} \in N_{c}(S, w)\right\} \\
& \geqslant \inf \left\{\left\|x^{*}+u^{*}\right\| \mid x^{*} \in D_{c}^{*}(w)\left(y^{*}\right), u^{*} \in N_{c}(S, w)\right\}-(k / \tau)
\end{aligned}
$$

or

$$
k / \tau \geqslant C^{*}\left(D_{c}^{*} F(w), N_{c}(S, w)\right) .
$$

If, in addition, $X$ has an equivalent Gâteaux differentiable norm, then (10) and the left inclusion in (7) imply in a similar way:

$$
k / \tau \geqslant C^{*}\left(D_{m}^{*} F(w), N_{m}(S, w)\right) .
$$

Generally, if there is an equivalent locally uniformly convex norm in $Y^{*}$ (while $\|\cdot\|$ may be different), then we shall get (11), (12), applying them subsequently to a sequence of such norms converging to $\|\cdot\|$.

The desired inequalities are obtained from (7), (8), (11), (12) precisely in the same way as in the two theorems above.

11.10. REMARK. If $\operatorname{dim} Y<\infty$, another surjection theorem follows from Corollary 6.3: if a bounded fan $\mathcal{Q}$ is an outer prederivative of $F$ at $z$ (i.e. $\left.F(z+h)-F(z) \in \mathbb{Q}(h)+r(h)\|h\| B_{Y}\right)$ and if $S$ is a closed convex cone, then $\omega(F, S, z)(t) \geqslant t(C(Q, S)-r(t))$. This result is akin to a theorem of Halkin [19] (for a more detailed comparison see [29]).

We also note that the situation with surjection theorems is very similar to what occurs in the classical smooth calculus: if the range space is finite-dimensional, we need only a derivative to establish the surjection property while, in the general case, we must either scan a neighbourhood or use strict derivatives.

We now proceed to consider certain applications including the Ljusternik type theorems, inverse mapping theorems and implicit function theorems.

11.11. Definition. Let, again, $F$ be a mapping from a neighbourhood of $z \in S \subset X$ into $Y$. Given a $y \in Y$, we denote $\mathcal{L}_{y}(F, S)=\{x \in S \mid F(x)=y\}$. The quantity

$$
\lambda(F, S, z)(t)=\inf \left\{\eta>0 \mid \rho\left(z, \mathcal{E}_{y}(F, S)\right)<\eta \text { whenever }\|y-F(z)\|<t\right\}
$$

will be called the modulus of regularity of $F$ on $S$ at $z$. Obviously $\lambda(F, S, z)(\cdot)$ is the minimal element in the collection of functions $\eta(\cdot)$ satisfying $\rho\left(z, \mathcal{E}_{y}(F, S)\right)<$ $\eta(\|y-F(z)\|)$. 
11.12. Proposition. $\lambda(F, S, z)(t)=\inf \{\tau>0 \mid \omega(F, S, z)(\tau)>t\}$. In particular, if $\omega(F, S, z)(t) \geqslant t c(c>0)$ for $0 \leqslant t \leqslant t_{0}$, then $\lambda(F, S, z)(t)<t / c$ for $0 \leqslant t<t_{0} / c$.

Proof. This follows directly from definitions.

Combining this result with the preceding ones, we can obtain various nonsmooth extensions of the theorem of Ljusternik which states that for a mapping $F$, strictly differentiable at $z$ and having a surjective derivative $F^{\prime}(z)$, the inequality $\rho\left(x, \mathcal{L}_{F(z)}(F)\right)<k\|F(x)-F(z)\|$ holds for certain $k>0$ and all $x$ sufficiently close to $z$.

We shall summarize some of such possible extensions in the following theorem.

11.13. Theorem. Let $F: X \rightarrow Y$ be defined on $S \cap U$ where $S$ is closed and $U$ open. Then the inequality

$$
\lambda(F, S, x)(t)<t / c
$$

is valid for all $x \in S, t>0$ such that $B(x, t+\tau) \subset U$ for certain $\tau>0$ (depending on $x$ ) under any of the following assumptions:

(A) $F$ is continuous and

$$
c=\inf _{u \in S \cap U} \lim _{\varepsilon \rightarrow 0} \operatorname{sl}\left(D_{\varepsilon}^{+} F(u), T(S, u)\right)>0 ;
$$

(B) $F$ is Lipschitz and either of $\left(\mathrm{B}_{1}\right)-\left(\mathrm{B}_{4}\right)$ is valid:

$\left(\mathrm{B}_{1}\right)$

$$
c=\inf _{u \in S \cap U} \sup _{\substack{\varepsilon>0 \\ \delta>0}} \frac{\operatorname{sl}\left(D_{e}^{+} F(u), T_{\delta}(S, u)\right)-L \delta}{1+\delta}>0
$$

$\left(B_{2}\right)$

$$
c=\sup _{\delta>0} \frac{\operatorname{Sl}\left(D_{t}^{0} F(x), T_{\delta}(S, x)\right)-L \delta-\mu(t, x)}{1+\delta}>0
$$

(where $L$ is the Lipschitz constant of $F$ and $\mu(t, x)$ is defined as $\mu(t)$ in 11.7 with $z$ replaced by $x$ and $x$ by $u$ ),

$\left(\mathrm{B}_{3}\right)$

$$
c=\inf _{u \in S \cap U} \lim _{\varepsilon \rightarrow 0} \operatorname{sl}^{*}\left(\left(D_{\varepsilon}^{0} F(u)^{*}, N_{c}(S, u)\right)>0,\right.
$$

$\left(\mathrm{B}_{4}\right)$ there is an equivalent Gâteaux differentiable norm in $X$ and

$$
c=\inf _{u \in S \cap U} s^{*}\left(D_{c}^{*} F(u), N_{m}(S, u)\right)>0
$$

(C) there is an equivalent locally uniformly convex norm in $Y^{*}$ and $c$ is as in any of the cases considered above but with $\mathrm{sl}$ and $\mathrm{Sl}$ replaced by $C, \mathrm{sl}^{*}$ replaced by $C^{*}$ and, in $\left(\mathrm{B}_{4}\right)$, with $D_{c}^{*}$ replaced by $D_{m}^{*}$.

The assumption (C) is fulfilled in particular if $\operatorname{dim} Y<\infty$ (cf. [24] in this connection where the case $Y=R$ was considered). For an alternative interpretation of results of such sort see [17]. 
11.14. TheOREM (INVERSE MAPPING THeOREM). Let $F$ be a Lipschitz mapping from $a$ neighbourhood of $z \in X$ into $Y$. Let $\sigma$ be the upper bound of those $t>0$ which satisfy

$$
\mathrm{Sl}\left(D_{t}^{0} F(z)\right)>0, \quad C^{*}\left(D_{t}^{0} F(z)\right)=C^{*}\left(D_{t}^{0} F(z),\{0\}\right)>0
$$

(and such that $B(z, t)$ belongs to the domain of $F$ ). Then for any $0<\tau<\sigma$ the restriction of $F$ to $U(z, \tau)$ is a Lipschitz homeomorphism onto an open neighbourhood of $F(z)$ with a Lipschitz constant of the inverse mapping not more than $\left(C^{*}\left(D_{\tau}^{0} F(z)\right)^{-1}\right.$.

Proof. It follows from 11.7 that $F(U(z, \tau))$ is a neighbourhood of $z$. To prove that it is an open set, take some $u$ belonging to $U(z, \tau)$, and let $\varepsilon>0$ be such that $\tau+\varepsilon<\sigma$. Then $D_{\varepsilon}^{+} F(u)(h) \subset D_{\tau+e}^{0} F(z)(h)$ for any $h$ so that

$$
\operatorname{sl}\left(D_{e}^{+} F(u),|\cdot|\right) \geqslant \operatorname{sl}\left(D_{\tau+e}^{0} F(z),|\cdot|\right)
$$

for any equivalent norm $|\cdot|$.

Choose a norm $|\cdot|$ sufficiently close to $\|\cdot\|$ and satisfying $\operatorname{sl}\left(D_{\tau+e}^{0} F(z),|\cdot|\right)=a$ $>0$. Then

$$
\operatorname{sl}\left(D_{e}^{+} F(u),|\cdot|\right)>a .
$$

This is true for any $u \in U(z, \tau)$ and Theorem 11.7 shows that, for any such $u$, the set $F(U(z, \tau))$ contains a neighbourhood of $F(u)$.

Now if $x, u \in U(z, \tau), y=F(x), v=F(u)$, then

$$
y-v=F(x)-F(u) \in D_{\tau}^{0} F(z)(x-u) ;
$$

hence $\|y-v\|>C^{*}\left(D_{\tau}^{0} F(z)\right)\|x-u\|>0$ or, in other words,

$$
\left\|F^{-1}(y)-F^{-1}(v)\right\| \leqslant\left(C^{*}\left(D_{\tau}^{0} F(z)\right)\right)^{-1}\|y-v\| \text {. }
$$

11.15. CoRollaRY. Let $F$ be a continuous mapping from a neighbourhood of $z$ into $Y$. Assume that there is a strict prederivative $\mathbb{Q}$ of $F$ at $z$ such that for some $t$

$$
\mathrm{Sl}(\mathbb{Q})>r(t), \quad C^{*}(\mathbb{Q})>r(t)
$$

(see 11.6 for the definition of $r(\cdot)$ ). Then for any $0<\tau<\sigma$ where $\sigma$ is the upper bound of such $t$, the restriction of $F$ on $U(z, \tau)$ is a homeomorphism onto an open neighbourhood of $F(z)$ and the inverse mapping is Lipschitz with constant not exceeding $\left(C^{*}(\mathscr{Q})-r(\tau)\right)^{-1}$.

Proof. This follows from the proof of the theorem. We leave it to the reader to adjust the proof as well as to derive alternative versions of the inverse mapping theorem connected with other criteria for surjection.

11.16. TheOREM (IMPLiCIT FunCtion TheOREM). Let $F$ be a continuous mapping from a neighbourhood of $\left(x_{0}, y_{0}\right) \in X \times Y$ into $W$. Assume that there is a fan $\mathbb{Q}$ from $Y$ into $W$ such that

$$
F(x, y+h)-F(x, y) \in \mathbb{Q}(h)+r(x, y, h)\|h\| B_{W},
$$

where $r(x, y, h) \rightarrow 0$ if $x \rightarrow x_{0}, y \rightarrow y_{0}, h \rightarrow 0$. If $\mathrm{Sl}(\mathscr{Q})>0$ and $C^{*}(\mathbb{Q})>0$, then there are neighbourhoods $U$ of $x_{0}, V$ of $y_{0}$ and a continuous mapping from $U$ into $Y$ such that relations $F(x, y)=F\left(x_{0}, y_{0}\right)$ and $y=f(x)$ are equivalent for $x \in U$, $y \in V$. If, in addition, $F$ is Lipschitz, then $f$ is also Lipschitz. 
Proof. Let

$$
r(t, s)=\lim _{\delta \rightarrow 0} \sup \left\{r(x, y, h) \mid\left\|x-x_{0}\right\|<t,\left\|y-y_{0}\right\|<s,\|h\|<\delta\right\} .
$$

Then $r(t, s) \rightarrow 0$ if $t \rightarrow 0, s \rightarrow 0$. Take $t>0$ and $s>0$ so small that $\mathrm{Sl}(\mathbb{Q})-r(t, s)$ $>0$ and $C^{*}(\mathbb{Q})-r(t, s)>0$. We can assume also, taking if necessary a suitable equivalent norm, that $\mathrm{sl}(\mathbb{Q})-r(t, s)>0$.

It is obvious that, for any fixed $x \in U\left(x_{0}, t\right)$,

$$
\inf _{\left\|y-y_{0}\right\|<s} \lim _{\delta \rightarrow 0} \operatorname{sl}\left(D_{y \delta}^{+} F(x, y)\right)>\operatorname{sl}(Q)-r(t, s)=c>0
$$

(because $D_{y \delta}^{+} F(x, y)(h) \subset \mathcal{Q}(h)+r(t, s)\|h\| B_{Y}, D_{y \delta}^{+} F(x, y)$ being the outer $\delta$-prederivative of $F(x, \cdot)$ at $y)$. Therefore, according to 11.5, $U\left(F\left(x, y_{0}\right), c s\right) \subset$ $F\left(x, U\left(y_{0}, s\right)\right)$. Let $0<\varepsilon<t$ be such that $\left\|F\left(x, y_{0}\right)-F\left(x_{0}, y_{0}\right)\right\|<c s$ whenever $\left\|x-x_{0}\right\|<\varepsilon$. Then $F\left(x_{0}, y_{0}\right) \subset F\left(x, U\left(y_{0}, s\right)\right)$ for any such $x$.

On the other hand, $F(x, \cdot)$ is one-to-one on $U\left(y_{0}, s\right)$ (this follows from 11.15 because $D_{y s}^{0} F\left(x, y_{0}\right)(h) \subset \mathbb{Q}(h)+r(t, s)\|h\| B_{W}$ for all $h$ and hence $C^{*}\left(D_{y s}^{0} F\left(x, y_{0}\right)\right)$ $\left.>C^{*}(\mathbb{Q})-r(t, s)\right)$.

Thus for any $x \in U=U\left(x_{0}, \varepsilon\right)$, there is precisely one $y \in V=U\left(y_{0}, s\right)$ such that $F(x, y)=F\left(x_{0}, y_{0}\right)$. Denote this $y$ by $f(x)$. It remains to prove that $f$ is continuous and Lipschitz if $F$ has these properties.

Let $x, u \in U$. Then

$$
\begin{aligned}
& 0=F(u, f(u))-F(x, f(x)) \\
& =F(u, f(u))-F(x, f(u))+F(x, f(u))-F(x, f(x)) \\
& \in F(u, f(u))-F(x, f(u))+D_{y s}^{0} F\left(x, y_{0}\right)(f(u)-f(x)) \\
& \text { or } F(x, f(u))-F(u, f(u)) \in D_{y s}^{0} F\left(x, y_{0}\right)(f(u)-f(x)) \text {; hence } \\
& \begin{aligned}
\|F(x, f(u))-F(u, f(u))\| & \geqslant C^{*}\left(D_{y s}^{0} F\left(x, y_{0}\right)\right)\|f(u)-f(x)\| \\
& >\left(C^{*}(\mathbb{Q})-r(t, s)\right)\|f(u)-f(x)\|
\end{aligned}
\end{aligned}
$$

which yields the desired conclusion.

Observe that we have been dealing in this proof with what can be called "partial" outer and strict prederivatives.

11.17. REMARK. There are other ways to derive implicit function theorems using what has been obtained before in this paper. In fact, this concerns all the other results of this section as well, including theorems on surjection which have been considered here more thoroughly than other results.

For instance, upon examining the proof of 11.9, it is not difficult to note that in the second part of $11.9\left(D_{e}^{0} F(x)\right)^{*}$ can be replaced by a weaker coderivative connected with $d^{+} f_{y^{*}}$ in the same way as $D_{m}^{*} F(x)$ relates to $d^{-} f_{y^{*}}$. This would enable us to consider outer prederivatives $\mathbb{Q}$ defined by sets $\mathfrak{A}$ of operators with which we can also associate dual objects

$$
\mathbb{Q}^{*}\left(y^{*}\right)=\left\{x^{*} \mid x^{*}=A^{*} y^{*} \text { for certain } A \in \mathfrak{X}\right\}
$$

and to prove a corresponding surjection theorem. Such a result would be closely related to results of Warga involving derivate containers which, as we have already mentioned, are strict prederivatives generated by (nonconvex, in general) sets of linear operators defined via approximation by smooth mappings. 
It also seems possible to prove surjection theorems involving weak outer prederivatives and so on.

12. Examples. In this section we shall calculate or estimate strict prederivatives, upper derivatives or $C$-coderivatives of several specific mappings without going too far into details. The last example gives an idea of how higher derivatives can be defined in the framework of the approach developed here.

12.1. Let $T$ be a compact metrizable space. Consider the following composition operator $F$ from $C^{m}(T)$ into $C^{n}(T)$ (the spaces of continuous mappings from $T$ into $R^{m}$ and $R^{n}$ respectively):

$$
F(x(\cdot))(t)=\varphi(t, x(t))
$$

where $\varphi(t, x)$ is a continuous function on $T \times R^{m}$ with values in $R^{n}$.

Let $z(\cdot) \in C^{m}(T)$ be given. We assume that there are $\delta>0$ and $k>0$ such that $\left\|\varphi(t, x)-\varphi\left(t, x^{\prime}\right)\right\| \leqslant k\left\|x-x^{\prime}\right\|$ if $\|x-z(t)\|<\delta,\left\|x^{\prime}-z(t)\right\|<\delta$. Then $F$ is Lipschitz in a neighbourhood of $z(\cdot)$ with constant $k$.

Let $s_{e}\left(t, q^{*}, h\right)=\varphi_{e}^{0}\left(t, z(t) ; q^{*}, h\right)\left(q^{*} \in R^{n}, h \in R^{m}\right)$. Then $s_{e}$ is continuous in $\left(q^{*}, h\right)$ and lower semicontinuous in $t$. If we denote by $Q_{e}(t)$ the strict $\varepsilon$-prederivative of $\varphi(t, \cdot)$ at $z(t)$, that is to say, the fan whose support function is $s_{e}(t, \cdot, \cdot)$, then for any $h(\cdot) \in C^{m}(t)$, the set-valued mapping $t \rightarrow \mathbb{Q}_{e}(t)$ is 1.s.c. By the selection theorem of Michael [40], for any $t_{0} \in T$ and any $y_{0} \in \mathbb{Q}_{e}\left(t_{0}\right)$, there is a continuous selection of this set-valued mapping which passes through $\left(t_{0}, y_{0}\right)$. Denote by $\mathcal{Q}(h(\cdot))$ the collection of all continuous selections of $Q_{e}(t)(h(t))$.

Then $Q$ is a bounded fan from $C^{m}(T)$ into $C^{n}(T)$. Boundedness and homogeneity of $\mathcal{Q}$ are obvious; it is also clear that the values of $\mathscr{Q}$ are closed and convex. Let us verify that $\mathbb{Q}$ satisfies (1.1.4) or, which is the same, that the support function of $\mathbb{Q}$ is convex in $h(\cdot)$.

To prove this, we first note that any $y^{*} \in\left(C^{n}(T)\right)^{*}$ can be represented in the form

$$
\left\langle y^{*}, y(\cdot)\right\rangle=\int_{T}\langle\xi(t), y(t)\rangle d \mu
$$

where $\mu$ is a probability Radon measure on $T$ and $\xi(\cdot)$ is a $\mu$-summable mapping from $T$ into $R^{n}$, and $\left\|y^{*}\right\|=\int_{T}\|\xi(t)\| d \mu$.

It is sufficient to prove convexity of $s_{Q}\left(y^{*}, h(\cdot)\right)$ only for those $y^{*}$ which correspond to continuous $\xi(\cdot)$ because such $y^{*}$ are norm dense in $Y^{*}=\left(C^{n}(T)\right)^{*}$. If $\xi(\cdot)$ is continuous, then the function $t \rightarrow s_{e}(t, \xi(t), h(t))$ is l.s.c. and we can find a sequence of continuous selections of $Q_{e}(t)(h(t))$ such that

$$
\sup _{k}\left\langle\xi(t), q_{k}(t)\right\rangle=s_{e}(t, \xi(t), h(t))
$$

for all $t$. Using standard unit partition techniques, we can, for any $\delta>0$, transform $\left\{q_{k}(\cdot)\right\}$ into another sequence $\left\{y_{k}(\cdot)\right\}$ (also formed by continuous selections) such that

$$
\left.\left\langle\xi(t), y_{k}(t)\right\rangle\right\rangle \max _{1<i<k-1}\left\langle\xi(t), q_{i}(t)\right\rangle-\delta \sum_{i=1}^{k-1} 2^{-i}
$$


and prove that

$$
s_{\mathbb{Q}}\left(y^{*}, h(\cdot)\right)=\int_{T} s_{\varepsilon}(t, \xi(t), h(t)) d \mu .
$$

It is easy to conclude from here that

$$
F^{0}\left(z(\cdot) ; y^{*}, h(\cdot)\right) \leqslant \int_{T} \varphi^{0}(t, z(t) ; \xi(t), h(t)) d \mu .
$$

Simple examples show that the upper derivative of $F$ does not necessarily exist. For instance, let $\varphi(t, x)=t \sin (x / t)$ if $t \neq 0$ and $\varphi(0, x)=0(T=[-1,1], m=n$ $=1)$. Then, if $z(t)=0$,

$$
\varphi_{x}^{\prime}(t, 0)= \begin{cases}1, & \text { if } t \neq 0 \\ 0, & \text { if } t=0\end{cases}
$$

and the corresponding multifunction has no continuous selections.

To summarize all said, we can assert that, more generally, if $t \rightarrow \mathbb{Q}(t)$ is a mapping from $T$ into $\mathscr{F}\left(R^{m}, R^{n}\right)$ such that

(i) $s_{\mathscr{Q}(t)}\left(q^{*}, h\right) \leqslant k\left\|q^{*}\right\|\|h\|$,

(ii) $s_{\mathscr{Q}(t)}\left(q^{*}, h\right)$ is 1.s.c. in $t$, and

(iii) $\varphi(t, x+h)-\varphi(t, x) \in \mathbb{Q}(t)(h)+r(t, x, h)\|h\| B_{R^{n}}$,

where $r(t, x, h) \rightarrow 0$ uniformly in $t$ if $x \rightarrow z(t)$ and $h \rightarrow 0$, then the set-valued mapping $h(\cdot) \rightarrow \mathbb{Q}(h(\cdot))$ defined by $\mathbb{Q}(h(\cdot))=\left\{y(\cdot) \in C^{n}(T) \mid y(t) \in \mathbb{Q}(t)(h(t))\right.$, $\forall t\}$ is a bounded fan which is a strict prederivative of $F$ at $z(\cdot)$ and

$$
s_{\mathbb{Q}}\left(y^{*}, h(\cdot)\right)=\int_{T} s_{\mathbb{Q}(t)}(\xi(t), h(t)) d \mu .
$$

The adjoint to $\mathbb{Q}$ is defined as follows: if $y^{*} \sim(\xi(\cdot), \mu)$, then $\mathbb{Q}^{*}\left(y^{*}\right)$ contains those $x^{*} \in\left(C^{m}(T)\right)^{*}$ which can be represented by pairs $(\eta(\cdot), \mu)$ such that $\eta(\cdot)$ is a measurable selection of the set-valued mapping $t \rightarrow \mathbb{Q}^{*}(t)(\xi(t))$.

12.2. Consider the same mapping as above but under a different set of assumptions. Specifically, we assume that $T$ is equipped with a fixed positive Radon measure $\lambda$ and

(a) for any $x \in R^{m}$, the mapping $\varphi(\cdot, x)$ is $\lambda$-summable;

(b) there exist $\varepsilon>0$ and a $\lambda$-summable positive function $k(t)$ such that

$$
\left\|\varphi(t, x)-\varphi\left(t, x^{\prime}\right)\right\|<k(t)\left\|x-x^{\prime}\right\|
$$

whenever $\|x-z(t)\|<\varepsilon,\left\|x^{\prime}-z(t)\right\|<\varepsilon$.

Then $F$ is a Lipschitz mapping from a neighbourhood of $z(\cdot) \in C^{m}(T)$ into $L_{1}\left(T, \lambda, R^{n}\right)$.

In this case the calculation is even simpler. The final result is the following: if $t \rightarrow \mathbb{Q}(t)$ is a mapping from $T$ into $\mathscr{F}\left(R^{m}, R^{n}\right)$ such that

(i) $\|\mathbb{Q}(t)\| \leqslant k(t)$ for all $t \in T$;

(ii) the set-valued mapping $t \rightarrow \mathbb{Q}(t)(h(t))$ is $\lambda$-measurable for any $h(\cdot) \in C^{m}(T)$;

(iii) $\varphi(t, x+h)-\varphi(t, x) \in \mathbb{Q}(t)(h)+r(t, x, h)\|h\| B_{R^{n}}$, where $r(t, x, h)>0$ and

$$
\lim _{\varepsilon \rightarrow 0} \int_{T} \sup \{r(t, x, h) \mid\|x-z(t)\|<\varepsilon,\|h\|<\varepsilon\} d \lambda=0,
$$


then $\mathbb{Q}(h(\cdot))=\left\{y(\cdot) \in L_{1}\left(T, \lambda, R^{n}\right) \mid y(t) \in \mathbb{Q}(t)(h(t)), \forall t\right\}$ is a bounded fan from $C^{m}(T)$ into $L_{1}\left(T, \lambda, R^{n}\right)$ which is a strict prederivative of $F$ at $z(\cdot)$.

Furthermore, for any $y^{*}(\cdot) \in L_{\infty}\left(T, \lambda, R^{n}\right)$, we have

$$
s_{\mathscr{Q}}\left(y^{*}(\cdot), h(\cdot)\right)=\int_{T} s_{\mathscr{Q}(t)}\left(y^{*}(t), h(t)\right) d \lambda
$$

and (cf. [11])

$$
F^{0}\left(z(\cdot) ; y^{*}(\cdot), h(\cdot)\right) \leqslant \int_{T} \varphi^{0}\left(t, z(t) ; y^{*}(t) ; h(t)\right) d \lambda,
$$

so that any element of $D^{0} F(z(\cdot))(h(\cdot))$ must be a measurable selection of the multifunction $t \rightarrow D^{0} \varphi(t, z(t))(h(t))$ and, for any $y^{*}(\cdot) \in L_{\infty}\left(T, \lambda, R^{n}\right)$, the corresponding value of the $C$-coderivative $D_{c}^{*} F(z(\cdot))$ must belong to the collection of functionals $x^{*} \in\left(C^{m}(T)\right)^{*}$ which can be represented in the form $\left\langle x^{*}, h(\cdot)\right\rangle=$ $\int_{T}\langle p(t), h(t)\rangle d \lambda$ where $p(\cdot)$ is $\lambda$-summable and $p(t) \in D_{c}^{*} \varphi(t, z(t))\left(y^{*}(t)\right)$ almost everywhere.

12.3. Let $T=[0,1], m=n$ and $\varphi$ be the same as above. Consider the following mapping from $C^{n}(T)$ into itself:

$$
F(x(\cdot))(t)=x(t)+\int_{0}^{t} \varphi(\tau, x(\tau)) d \tau,
$$

i.e. the composition of the one considered in 12.2 and a linear mapping. It is not difficult to write all formulas using 12.2.

If $\mathcal{Q}(t)$ satisfies conditions (i)-(iii) of 12.2 , then

$$
\mathscr{C}(h(\cdot))=\left\{y(\cdot) \in C^{n}(T) \mid y(t) \in h(t)+\int_{0}^{t} \mathscr{Q}(\tau)(h(\tau)) d \tau\right\}
$$

is a strict prederivative of $F$ at $z(\cdot)$ and, for any $y^{*} \sim(\xi(\cdot), \mu) \in\left(C^{n}(T)\right)^{*}$,

$$
\begin{aligned}
s_{\mathscr{Q}}\left(y^{*}, h(\cdot)\right) & =\int_{0}^{1}\left[\langle\xi(t), h(t)\rangle d \mu+s_{\mathscr{Q}(t)}\left(\int_{t}^{1} \xi(t) d \mu, h(t)\right)\right] d t, \\
F^{0}\left(z(\cdot) ; y^{*}, h(\cdot)\right) & \leqslant \int_{0}^{1} \varphi^{0}(t, z(t) ; \xi(t), h(t)) d \mu
\end{aligned}
$$

and $D_{c}^{*} F(z(\cdot))\left(y^{*}\right)$ belongs to the collection of those $x^{*} \in\left(C^{n}(T)\right)^{*}$ which can be represented in the form:

$$
\left\langle x^{*}, h(\cdot)\right\rangle=\int_{0}^{1}\langle\xi(t), h(t)\rangle d \mu+\int_{0}^{1}\langle\eta(t), h(t)\rangle d t,
$$

where $\eta(t)$ is a measurable selection of the set-valued mapping $t \rightarrow$ $\mathbb{Q}^{*}(t)\left(\int_{t}^{1} \xi(\tau) d \mu\right)$.

As follows from (1), $F$ has a strict prederivative with norm compact values; in particular $D^{0} F(z(\cdot))$ exists. This, in turn, shows that the mapping considered in 12.2 also has an upper derivative at $z(\cdot)$. Moreover, it can be shown that in the case considered now, the upper derivative is also a strict prederivative, obviously, the minimal one.

Another important property of $F$ is that, whenever a strict prederivative $\mathbb{Q}$ of $F$ at $z(\cdot)$ is defined by $(1)$, we have $C(\mathscr{Q})>0$ and $C^{*}(\mathscr{Q})>0$ so that (since any separable Banach space has an equivalent norm with a strictly convex dual [2]), $F$ is necessarily a Lipschitz homeomorphism about $z(\cdot)$. 
Let us estimate $C(\mathscr{Q})$ and $C^{*}(\mathscr{Q})$. Let $y^{*} \sim(\xi(\cdot), \mu) \in\left(C^{n}(T)\right)^{*}$ be given. We must verify that there is $c>0$ independent of $y^{*}$ such that

$$
\inf _{\|h(\cdot)\|<1}\left(\int_{0}^{1}\langle\xi(t), h(t)\rangle d \mu+\int_{0}^{1} s_{\mathscr{Q}(t)}\left(\int_{t}^{1} \xi(\tau) d \mu, h(t)\right) d t\right)<-c\left\|y^{*}\right\| .
$$

In view of condition (i) of 12.2 , we can be sure that this quantity cannot be greater than

$$
\inf _{\|h(\cdot)\|<1}\left(\int_{0}^{1}\langle\xi(t), h(t)\rangle d \mu+\int_{0}^{1} k(t)\|h(t)\|\left(\int_{t}^{1}\|\xi(\tau)\| d \mu\right) d t\right) .
$$

On the other hand, the latter quantity will not change if we extend the calculation of the lower bound to all $h(\cdot) \in L_{\infty}$ with $\|h(\cdot)\|_{\infty} \leqslant 1$. Therefore the lower bound we are trying to estimate cannot be greater than

$$
\inf \left(-\int_{0}^{1}\|\xi(t)\| \chi(t) d \mu+\int_{0}^{1} k(t) \chi(t)\left(\int_{t}^{1}\|\xi(\tau)\| d \mu\right) d t\right)
$$

where the infimum is being sought on the collection of characteristic functions $\chi(t)$ of measurable subsets of $[0,1]$.

Changing variables in this integral, we can express the quantity as follows:

$$
\inf \left(-\int_{0}^{1}\left(\|\xi(t)\| \chi(t)-\|\xi(t)\| \int_{0}^{t} k(\tau) \chi(\tau) d \tau\right) d \mu\right) .
$$

Since $k(\cdot)$ is summable, we can find $\delta>0$ such that $\int_{E} k(t) d t<\frac{1}{2}$ whenever mes $E<\delta$ (mes stands for the Lebesgue measure). Find $E \subset[0,1]$ with mes $E<\delta$ and such that

$$
\int_{E}\|\xi(t)\| d \mu>\delta\left\|y^{*}\right\|=\delta \int_{0}^{1}\|\xi(t)\| d \mu
$$

(which is, of course, possible) and let $\chi(t)$ be the characteristic function of $E$. Then we see that $(2)$ is not greater than

$$
-\int_{E}(\|\xi(t)\|-(1 / 2)\|\xi(t)\|) d \mu<-(\delta / 2)\left\|y^{*}\right\| .
$$

It remains to set $c=\delta / 2$.

To show that $C^{*}(\mathscr{Q})$ is positive, we must prove the existence of $c>0$ such that

$$
\inf \left\{\|y(\cdot)\| \mid y(\cdot) \in C^{n}(T), y(t) \in h(t)+\int_{0}^{t} \mathscr{Q}(\tau)(h(\tau)) d \tau\right\}>c\|h(\cdot)\| .
$$

If such a $c$ does not exist, then there are sequences $\left\{h_{m}(\cdot)\right\} \subset C^{n}(T)\left(\left\|h_{m}(\cdot)\right\|=1\right)$ and $\left\{q_{m}(\cdot)\right\} \subset L_{1}\left(T, R^{n}\right)\left(q_{m}(t) \in \mathbb{Q}(t)\left(h_{m}(t)\right)\right.$ almost everywhere) such that $y_{m}(t)$ $=h_{m}(t)+\int_{0}^{t} q_{m}(\tau) d \tau$ converges to zero uniformly.

Since $\left\|q_{m}(t)\right\|<k(t)$ for all $t$, usual compactness arguments show that (at least a subsequence of) $\left\{h_{m}(\cdot)\right\}$ converges uniformly to some $h(\cdot),\|h(\cdot)\|=1$, and $\left\{q_{m}(\cdot)\right\}$ converges in $L_{1}$ to some $q(\cdot)$ such that $q(t) \in \mathbb{Q}(t)(h(t))$ almost everywhere.

It follows that $\|h(t)\|-\int_{0}^{t} k(t)\|h(t)\| d t<0$ for all $t$ which may be only if $h(t) \equiv 0$. 
12.4. Here we shall briefly discuss how the approach developed in this paper can be applied to higher derivatives. We hope to consider it in greater detail elsewhere. Let $F$ be a mapping from a neighbourhood of $z \in X$ into $Y$. An $n$-fan (see 1.14) $Q$ from $X^{n}$ into $Y$ will be called the $n$th outer prederivative of $F$ at $z$ if, for any $i=1, \ldots, n-1$, there is an $i$-linear bounded mapping $A_{i}: X^{i} \rightarrow Y$ such that

$$
\begin{gathered}
F(z+h)-F(z) \in A_{1} h+(1 / 2) A_{2}(h, h)+\cdots+(1 /(n-1) !) A_{n-1}(h, \ldots, h) \\
+(1 / n !) \mathcal{Q}(h, \ldots, h)+r(h)\|h\|^{n} B_{Y},
\end{gathered}
$$

where $r(h) \rightarrow 0$ if $\|h\| \leqslant 0$.

If $F$ is $(n-1)$ times continuously differentiable at $z$ and the $(n-1)$ st derivative of $F$ at $z$ is Lipschitz, then $Q$ can be defined, say, as the strict $\varepsilon$-prederivative of $F^{(n-1)}$ at $z$.

A similar definition (for second derivatives) was also offered by Rockafellar who drew my attention to the fact that it is possible to consider only symmetric 2-fans as candidates for second order prederivatives.

Consider a particular case of a real-valued function $f$. Let $\mathcal{Q}$ be a second outer prederivative of $f$ at $z$. Then, as it is easy to see,

$$
\begin{aligned}
& f(z+h) \leqslant f(z)+\left\langle f^{\prime}(z), h\right\rangle+(1 / 2) s_{\mathfrak{Q}}(1, h, h)+o\left(\|h\|^{2}\right), \\
& f(z+h) \geqslant f(z)+\left\langle f^{\prime}(z), h\right\rangle-(1 / 2) s_{\mathbb{Q}}(1,-h, h)+o\left(\|h\|^{2}\right) .
\end{aligned}
$$

It follows that a necessary condition for $f$ to attain a local minimum at $z$ is $\left(f^{\prime}(z)=0\right.$ and) $s_{\mathbb{Q}}(1, h, h) \geqslant 0$ for all $h$, and a sufficient condition (if $f^{\prime}(z)=0$ ) is that there exist $k>0$ such that $s_{\mathscr{Q}}(1,-h, h)<-k\|h\|^{2}$.

Thus it is natural to call a 2 -fan $\mathbb{Q}$ from $X \times X$ into $R$ positively semidefinite if $s_{\mathbb{Q}}(h, h) \geqslant 0$ for all $h$ and positively definite if $s_{\mathbb{Q}}(1,-h, h)<-k\|h\|^{2}$ for some $k>0$ and all $h$. Note that in the latter case $s_{\mathbb{Q}}(1, h, h)>k\|h\|^{2}$ (because of the convexity of $s_{\mathfrak{Q}}$ in each argument) but this condition is weaker than positive definiteness.

\section{BIBLIOGRAPHY}

1. G. P. Akilov and S. S. Kutateladze, Ordered vector spaces, "Nauka", Novosibirsk, 1978.

2. D. Amir and J. Lindenstrauss, The structure of weakly compact sets in Banach spaces, Ann. of Math. 88 (1968), 35-46.

3. J. P. Aubin, Contingent derivatives of set-valued maps and existence of solutions to differential inclusions, MRS Technical Summary Report, Univ. Wisconsin-Madison, 1979.

4. Derivatives and codifferentials of maps with closed convex graphs and convex operators, MRS Technical Summary Report, Univ. Wisconsin-Madison, 1979.

5. W. Bonnice and R. J. Silverman, The Hahn-Banach extension and the least upper bound properties are equivalent, Proc. Amer. Math. Soc. 18 (1967), 843-850.

6. N. Bourbaki, Espaces vectoriels topologiques, Hermann, Paris, 1955.

7.

8. F. H. Clarke, Generalized gradients and applications, Trans. Amer. Math. Soc. 205 (1975), 245-262.

9. __ On the inverse function theorem, Pacific J. Math. 62 (1976), 97-102.

10. A A new approach to Lagrange multipliers, Math. Oper. Res. 1 (1976), 165-174.

11. Generalized gradients of Lipschitz functions (to appear).

12. __ Nonsmooth analysis and optimization, Proc. Internat. Congr. Math. (Helsinki, 1978), Acad. Sci. Fenn, 1980, pp. 847-853.

13. H. H. Corson and J. Lindenstrauss, Contimuous selections with nonmetrizable range, Trans. Amer. Math. Soc. 121 (1966), 492-504. 
14. S. Dolecki, $A$ general theory of necessary optimality conditions (to appear).

15. S. Dolecki and S. Rolewicz, Exact penalty for local minima, SIAM J. Control Optim. 17 (1979), 596-606.

16. I. Ekeland, On the variational principle, J. Math. Anal. Appl. 47 (1974), 324-353.

17. , Nonconvex minimization problems, Bull. Amer. Math. Soc. 1 (1979).

18. H. Halkin, Mathematical programming without differentiability, Calculus of Variations and Control Theory, Academic Press, New York, 1976.

19. Necessary conditions for optimal control problems with differentiable and nondifferentiable data, Lecture Notes in Math., vol. 68, Springer-Verlag, Berlin and New York, 1978, pp. 77-118.

20. H. B. Hiriart-Urruty, Théorèmes de valeur moyenne en analyse non-différentiable: cas de fonction $\dot{a}$ valeurs réels, C. R. Acad. Sci. Paris 287 (1978), 707-709.

21. Théorèmes de valeur moyenne en analyse non-différentiable: cas de fonction localement lipschitzienne à valeurs vectorielles, C. R. Acad. Sci. Paris 287 (1978), 751-753.

22. , Tangent cones, generalized gradients and mathematical programming in Banach spaces, Math. Oper. Res. 4 (1979), 63-82.

23. __ Mean value theorem in nonsmooth analysis, Numer. Funct. Anal. Optim. 2 (1980), 1-30.

24. A. D. Ioffe, Regular points of Lipschitz functions, Trans. Amer. Math. Soc. 251 (1979), 61-69.

25. __ Différentielles généralisées d'applications localement lipschitziennes d'un espace de Banach dans un autre, C. R. Acad. Sci. Paris 289 (1979), 637-640.

26. Necessary and sufficient conditions for a local minimum, SIAM J. Control Optim. 17 (1979), 245-288.

27. __ On foundations of convex analysis, Ann. New York Acad. Sci. 337 (1980), 103-118.

28. A new proof of the equivalence of the Hahn-Banach extension and the least upper bound properties, Proc. Amer. Math. Soc. (to appear).

29. , Necessary conditions in nonsmooth optimization, Math. Oper. Res. (to appear).

30. A. D. Ioffe and V. M. Tihomirov, Theory of extremal problems, North-Holland, Amsterdam, 1979.

31. A. D. Ioffe and V. L. Levin, Subdifferentials of convex functions, Trans. Moscow Math. Soc. 26 (1972), 1-72.

32. M. A. Krasnosel'skii and P. P. Zabreiko, Geometrical methods in nonlinear analysis, "Nauka", Moscow, 1975.

33. A. Ja. Kruger and B. Sh. Mordukhovich, Extremal points and Euler equations for nonsmooth optimization problems, Dokl. Akad. Nauk BSSR (to appear).

34. S. S. Kutateladze, Moduli which admit convex analysis, Dokl. Akad. Nauk SSSR 252 (1980), 789-792.

35. P. J. Laurent, Approximation et optimization, Hermann, Paris, 1972.

36. G. Lebourg, Valeur moyenne pour gradient généralisée, C. R. Acad. Sci. Paris 281 (1976), 795-797.

37. Yu. Linke, Sublinear operators with values in spaces of continuous functions, Dokl. Akad. Nauk SSSR 228 (1976), 540-542.

38. G. G. Magaril-Il'aev, Implicit function theorem for Lipschitz mappings, Uspehi Mat. Nauk 33 (1) (1978), 221-222.

39. M. D. Mesarovic and Yasuhiko Takahara, General system theory: mathematical foundations, Academic Press, New York, 1975.

40. E. Michael, Continuous selections. I, Ann. of Math. 63 (1956), 361-382.

41. H. Methlouthi, Calcul différéntiel multivoque, Cahiers Math. de la Decision, Univ. Paris IX, exposé $7702,1977$.

42. G. Sh. Mordukhovich, Maximum principle in the optimal time control problem with nonsmooth constraints, Prikl. Mat. Meh. 40 (1976), 1014-1023.

43. Metric approximations and necessary optimality conditions for general classes of nonsmooth extremal problems, Dokl. Akad. Nauk SSSR 254 (1980), 1072-1075.

44. L. Nachbin, A theorem of the Hahn-Banach type for linear transformations, Trans. Amer. Math. Soc. 68 (1950), 28-46.

45. J. P. Penot, Calcul sous-différentiel et optimisation, J. Funct. Anal. Appl. 27 (1978), 248-276.

46. B. H. Pourciau, Analysis and optimization of Lipschitz continuous mappings, J. Optim. Theory Appl. 22 (1977), 311-351.

47. S. M. Robinson, Regularity and stability theorems for convex multivalued functions, Math. Oper.

Res. 1 (1976), 130-142.

48. R. T. Rockafellar, Convex analysis, Princeton Univ. Press, Princeton, N. J., 1970. 
49. , Clarke's tangent cones and boundaries of closed sets in $R^{n}$, Nonlinear Anal. 3 (1979),

$145-154$.

50. Generalized directional derivatives and subgradients of nonconvex functions, Canad. J. Math. 32 (1980).

51. B. Rodrigues-Salinas and L. Bou, A Hahn-Banach theorem for arbitrary vector spaces, Boll. Un. Mat. Ital. (4) 10 (1974), 390-393.

52. N. Shor, Minimization methods for nondifferentiable functions and their applications, "Naukova Dumka", Kiev, 1979.

53. L. Thibault, Subdifferentials of compactly Lipschitzian vector-valued functions, Sem. d'Analyse Convex, Univ. du Languedoc, 8 (1978), exposé no. 5.

54. Mathematical programming and optimal control problems defined by compactly Lipschitzian mappings, Sem. d'Analyse Convex, Univ. du Languedoc, 8 (1978), exposé no. 10.

55. T. O. To, The equivalence of the least upper bound property and the Hahn-Banach extension property in ordered vector spaces, Proc. Amer. Math. Soc. 30 (1971), 287-296.

56. J. Warga, Derivative containers, inverse functions and controllability, Calculus of Variations and Optimal Control, Academic Press, New York, 1976.

57. Controllability and necessary conditions in unilateral problems without differentiability, SIAM J. Control Optim. 14 (1976), 546-573.

58. Controllability and a multiplier rule for nondifferentiable optimization problems, SIAM J. Control Optim. 16 (1978), 803-812.

59. , On bounding, interior and covering functions (to appear).

60. ___ Fat homeomorphisms and unbounded derivate containers (to appear).

61. A. G. Kusraev, On necessary conditions for an extremum for nonsmooth vector-valued mappings, Dokl. Akad. Nauk SSSR 242 (1978), 44-47.

62. E. S. Levitin, A. A. Miljutin and N. P. Osmolovskii, Higher order conditions for a local minimum in problems with constraints, Uspehi Mat. Nauk 33 (1978), 85-148.

Profsoyusnaya 85-1-203, Moscow 117279, U.S.S.R. 